\title{
Maximum power point of piezoelectric energy harvesters: a review of optimality condition for electrical tuning
}

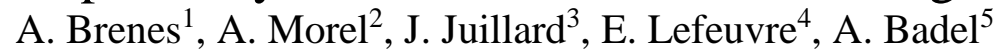 \\ ${ }^{1}$ Institut Supérieur d'Electronique de Paris (ISEP), 28 rue Notre Dame des Champs, 75006, \\ Paris, France \\ ${ }^{2}$ Université Grenoble Alpes, CEA, LETI, MINATEC, F-38000 Grenoble, France \\ ${ }^{3}$ GeePs | Group of electrical engineering - Paris, UMR CNRS 8507, CentraleSupélec, Univ. \\ Paris-Sud, Sorbonne Universités, UPMC Univ. Paris 06, 3,11 rue Joliot-Curie, Plateau de \\ Moulon F-91192 Gif-sur-Yvette CEDEX \\ ${ }^{4}$ Centre for Nanoscience and Nanotechnology, Univ. Paris Sud - CNRS, \\ Université Paris-Saclay, France \\ ${ }^{5}$ SYMME, Université Savoie Mont-Blanc, France
}

\begin{abstract}
This paper is a review and synthetic overlook of existing energy harvesting circuits and techniques for piezoelectric energy scavengers. We provide a hierarchical presentation based on functional analysis to distinguish between existing solutions which may look superficially similar but prove to perform very differently in practice. Based on this hierarchical presentation, definitions of topologies, architectures and techniques are given in order to avoid redundancy among existing and future solutions. Then, after a thorough and unified mathematical analysis of the general problem to address, we present a comparison of the conditions for each technique to maximize the power flow from an external vibration source to an electrical load. This analysis is meant to help researchers and engineers make optimal choices for effective implementation of Maximum Power Point Tracking (MPPT).
\end{abstract}

\section{Introduction}

From an industrial perspective, the replacement of batteries by energy harvesters to power microsystems requires improvements in device size, weight, cost and performance. Among the energy sources available in the environment, several possibilities can be explored. For example, piezoelectric transducers scavenge mechanical energy from external vibrations with a remarkable power density [1]. Electromagnetic waves can also be exploited for wireless power transfer and the resulting optimization problems are similar $[2,3]$.

Scientific literature investigates many approaches to maximize the power harvested by piezoelectric energy harvesting systems and/or their bandwidth. Some studies are based on material science and/or transducer structure optimization [4]. A very detailed review of these improvements has recently been published [5]. However, system-level optimization requires a more general approach including also the optimization of the electrical interface. From that perspective, previous interesting works have compared existing harvesting techniques. For instance, one can refer to the very accurate analysis of Dicken et al. [6] who compared techniques which existed in 2012. However, this study was limited to weakly-coupled generators where the electrical load had no impact on the input current, for purposes of simplicity and clarity. The number of harvesting techniques has exploded in the last decade, and this assumption falls short in most new solutions, which exhibit very different performances depending on the piezoelectric generator characteristics. An interesting attempt to sum up the main conclusions about existing architectures has been published in 2018 [7]. This article describes and explains qualitatively resonant and non-resonant circuits. Interesting though it may be, it does not reconcile circuit-oriented approaches with analytical considerations about 
coupled generators like the ones of Halvorsen et al. or Hosseinloo et al. [8] [9]. For example, it reports a system from the literature that surpasses the theoretical limit [8] [9]. A recent study has summed up the main theoretical results to achieve optimal impedance matching but does not study the particular circumstances of the numerous existing architectures [10]. The aim of our paper is to propose a structured overview of existing techniques for piezoelectric energy harvesting $(\mathrm{PEH})$ and reconcile $\mathrm{PEH}$ circuits and theory in terms of optimal operating conditions - taking into account the bidirectional electromechanical coupling.

Previous studies have analyzed the optimal power that one can hope to achieve with piezoelectric transducers under different operating conditions. The conditions of study include monochromatic vibration, either constrained or unconstrained [8], broadband random vibrations [11] or general transient regime motions [12]. Unfortunately, the relationship between this theory and the practical implementations of electrical interfaces is not always straightforward. Therefore, theoretical studies, though useful, are of quite limited practical help when making a technological choice between existing architectures. At the other end of the spectrum, many studies have focused on maximizing the harvested power with dedicated electrical interfaces on a case-by-case basis [13] [14] [15]. Nevertheless, an overview is missing and, when confronted with the choice of an energy harvesting architecture, one may wonder which solution they should opt for.

Numerous works exist in scientific literature, bringing advances in both the theoretical understanding of optimal operating conditions and the practical implementation of an optimized harvesting technique with Maximum Power Point Tracking (MPPT). One of the first successful attempts to design an optimized piezoelectric energy harvester was proposed by Ottman et al. and included a load matching stage (also called load adaptation) [16, 17]. It proved that maximum power-point tracking techniques based on impedance matching is possible in order to optimize the power flow, from the generator to the storage element or the supplied circuit. A DC-DC buck-boost converter operated in discontinuous mode can for example perform such resistive matching with an adjustable duty-cycle and/or switching frequency [17]. Allintegrated versions of such MPPT have even been implemented recently [18].

One of the problems of the architecture proposed by Ottman et al. is that resistive impedance matching is not sufficient to extract the maximum power for all kinds of resonators. In particular, some generators would require a complex (and not just real) impedance matching to reach the maximum available power [19]. For this purpose, the use of an inductor constantly connected to the piezoelectric element is generally not considered because of the very large required inductance values - typically in the range of tenth of Henrys - leading to bulky and lossy inductors [20]. This statement has justified many works on nonlinear energy harvesting circuits able to perform such an optimization. Amongst existing topologies to perform complex impedance matching, it was shown that a small inductor connected with an electronic switch to the piezoelectric device may significantly improve energy conversion.

The nonlinear technique called SECE for "Synchronous Electric Charge Extraction" [21] was derived from the so-called "SSD" technique ("Synchronous Switch Damping") initially designed to damp undesired vibrations $[22,20]$. In this technique, energy was extracted synchronously with the piezoelectric oscillation and then transferred to an electrical storage cell. The initial idea of SECE is to leave the generator in open circuit most of the time, with the appropriate voltage polarity enabling to maximize energy conversion, and concentrate energy extraction within a very short instant where the current and voltage conditions are optimal. One of the main interests of this architecture is the independence between the harvested power and the load connected to the energy harvesting circuit. However, this relatively simple technique 
performs poorly for strongly coupled piezoelectric resonators [21]. For weakly-coupled resonators, it performs better than the initial architecture of Ottman et al., but can still not reach optimal impedance matching. Since the aforementioned publication, plenty works have proposed modifications to the original circuit [23] [24] [25] in order to improve the performance of classical SECE in terms of maximum power and/or bandwidth. Following this trend, the Phase-Shift SECE (PSSECE) technique has proven its ability to enlarge the bandwidth of strongly-coupled energy harvesters [25].

As alternatives to SECE, Guyomar and his colleagues have also proposed other nonlinear circuits to perform complex impedance matching, like parallel [26, 27] and series [28, 29] Synchronized Switch Harvesting on Inductor (P-SSHI and S-SSHI). These circuits take advantage of an additional stage including an inductor and a switch, triggered synchronously with the piezoelectric voltage. These solutions have raised interest until recently [30, 31] because of their good performances especially for weakly-coupled generators. Recent improvements to the technique propose an inductor-less circuit replacing the inductor with a sequence of synchronized switches, calling the resulting method SSHC [32, 33, 34].

All the aforementioned architectures are based on either full-bridge or half-bridge rectifiers. Integrated versions also exist for some of these techniques [35]. Following [36], other recent studies have replaced these bridge rectifiers by a shunt diode and studied the impact resulting from this change on the electromechanical energy conversion [37] [38]. Not only do such shuntdiode architectures improve the electrical power efficiency at low voltage levels but they also retroactively increase, under specific conditions, the amount of mechanical energy transferred into mechanical motion of the seismic mass, which brings larger amounts of energy into the electrical circuit.

Finally, a last category of architectures allows to get rid of either shunt or bridge diode rectifiers but at the cost of using coupled inductors. The first proposal of such an architecture for piezoelectric energy harvesters was initially called SSHI-MR [39] and then renamed OSECE (Optimized Synchronous Electric Charge Extraction) after small modifications in following works [40]. The technique lies in the use of a coupled inductor directly into the rectifying structure, so that the system harvests power in a coupled circuit via mutual inductances when the switch(es) is (are) closed. A further improvement of this method has recently been proposed and called SECPE (Synchronized Electric Charge Partial Extraction) [41]. The idea of SECPE is to tune the duration of charge extraction in an OSECE system. Adjusting the duration of charge extraction is one of the possible ways to match the impedance connected to the piezoelectric generator.

Between all these proposals, several significant differences appear but, since the corresponding electronic architectures sometimes look alike at first sight, a non-expert may have a hard time making a difference and grasping the real advantages.

Whatever the architecture and technique, Maximum Power Point Tracking (MPPT) requires the optimal control of one or more tuning parameters with respect to the frequency of the mechanical oscillation (and sometimes the oscillation level) to emulate the real and/or imaginary part of the load impedance [13, 19, 42, 43]. For example, in PSSECE, the tuning parameter is the instant of charge extraction. In tunable SECE and SECPE, it lies in the duration of charge extraction. In parallel- and series-SSHI, as well as in load adaptation, the optimized parameter is the output impedance. Based on the number of tuning parameters, the number of functional blocs (stages) they require and their architecture, existing techniques are categorized in Figure 1. Considering the large diversity of acronyms under use in piezoelectric energy 
harvesting, we also report, in Table 1, a glossary of the acronyms found in previous literature, along with their signification. For the sake of readability, we do not detail the differences between all these acronyms in this introduction. When required, their meaning is given in the following sections.

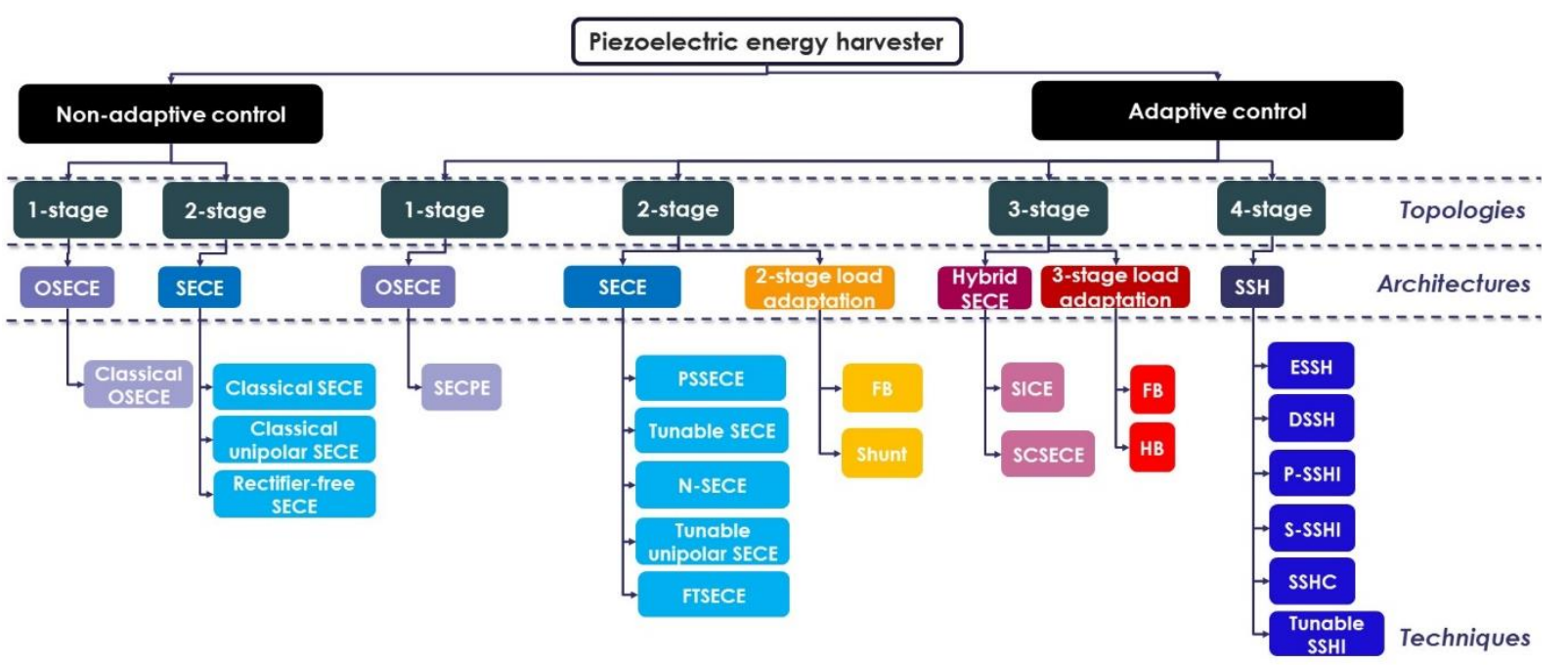

Figure 1: Existing strategies, topologies, architectures and techniques for piezoelectric energy harvesting.

TABLE 1. CLASSIFIED TABLE OF ACRONYMS FROM PREVIOUS LITERATURE

\begin{tabular}{|c|c|}
\hline Acronym & Signification \\
\hline \multicolumn{2}{|r|}{ General acronyms } \\
\hline $\begin{array}{c}\text { FOM } \\
\text { MPPT } \\
\text { PEH } \\
\text { SDOF } \\
\text { SSD }[22,20] \\
\end{array}$ & $\begin{array}{cc}\text { Figure Of Merit } \\
\text { Maximum Power Point Tracking } \\
\text { Piezoelectric Energy Harvester } \\
\text { Single Degree Of Freedom } \\
\text { Synchronized Switch Damping } \\
\end{array}$ \\
\hline \multicolumn{2}{|r|}{ OSECE and derivatives } \\
\hline \multicolumn{2}{|r|}{ Architecture acronyms } \\
\hline OSECE [40] & Optimized Synchronized Electric Charge Extraction \\
\hline SSHI-MR [39] & $\begin{array}{l}\text { Synchronized Switch Harvesting on Inductor with Magnetic Rectifier (later } \\
\text { renamed OSECE) }\end{array}$ \\
\hline \multicolumn{2}{|r|}{ Acronyms for techniques } \\
\hline (C)SPOSECE [44] & $\begin{array}{l}\text { (Compact) Self-Powered Optimized Synchronized Electric Charge } \\
\text { Extraction }\end{array}$ \\
\hline \multicolumn{2}{|r|}{ SECE and derivatives } \\
\hline \multicolumn{2}{|r|}{ Architecture acronyms } \\
\hline $\begin{array}{l}\text { SCE [45] [46] } \\
\text { SECE [21] }\end{array}$ & $\begin{array}{c}\text { Synchronized Charge Extraction (abbreviation of SECE) } \\
\text { Synchronized Electric Charge Extraction }\end{array}$ \\
\hline \multicolumn{2}{|r|}{ Acronyms for techniques } \\
\hline FTSECE [19] [47] & Frequency-Tuning Synchronized Electric Charge Extraction \\
\hline MS-SECE [48] [49] & Multiple-Shot Synchronized Electric Charge Extraction \\
\hline N-SECE [50] & Synchronized Electric Charge Extraction with $\mathrm{N}$ extraction events by period \\
\hline PSSECE [25] & Phase-shifted Synchronized Electric Charge Extraction \\
\hline SECPE [41] & Synchronized Electric Charge Partial Extraction \\
\hline Tunable SECE [24] & Tunable Synchronized Electric Charge Extraction \\
\hline $\begin{array}{l}\text { Tunable USECE [51] } \\
\text { USECE [38] }\end{array}$ & $\begin{array}{c}\text { Tunable Unipolar Synchronized Electric Charge Extraction } \\
\text { Unipolar SECE }\end{array}$ \\
\hline \multicolumn{2}{|r|}{ Hybrid SECE and derivatives } \\
\hline & Architecture acronym \\
\hline
\end{tabular}




\begin{tabular}{|c|c|}
\hline Hybrid SECE [52] & Hybrid Synchronized Electric Charge Extraction \\
\hline \multicolumn{2}{|r|}{ Acronyms for techniques } \\
\hline SICE [52] & Synchronous inversion and charge extraction \\
\hline SC-SECE [53] & Short-Circuited Synchronized Electric Charge Extraction \\
\hline \multicolumn{2}{|r|}{ SSH and derivatives } \\
\hline \multicolumn{2}{|r|}{ Architecture acronym } \\
\hline SSH [26] & Synchronized Switch Harvesting \\
\hline \multicolumn{2}{|r|}{ Acronyms for techniques } \\
\hline p-SSHI or PSSHI [26] & Parallel Synchronized Switch Harvesting on Inductor \\
\hline s-SSHI or SSSHI [28] & Series Synchronized Switch Harvesting on Inductor \\
\hline DSSH [54] & Double synchronized switch harvesting \\
\hline ESSH [55] & Enhanced Synchronized Switch Harvesting \\
\hline SSHC [32] & Synchronized Switch Harvesting on Capacitors \\
\hline \multicolumn{2}{|c|}{ Optimizations and/or autonomous implementations acronyms } \\
\hline COV-PSSHI [56] & COntrolable Voltage Parallel Synchronized Switch Harvesting on Inductor \\
\hline H-S ${ }^{3} \mathrm{HI}[57]$ & $\begin{array}{c}\text { High-frequency-included Surge-Inducing Synchronized-Switch Harvesting } \\
\text { on Inductor }\end{array}$ \\
\hline ISP-PSSHI [58] & $\begin{array}{c}\text { Improved Self-Powered Parallel Synchronized Switch Harvesting on } \\
\text { Inductor }\end{array}$ \\
\hline P-S3BF [59] & Parallel Synchronized Triple Bias-Flip \\
\hline PV-P-S3BF [60] [45] & Phase-variable Parallel Synchronized Triple Bias-Flip \\
\hline SP-PSSHI [61] & Self-Powered Parallel Synchronized Switch Harvesting on Inductor \\
\hline SP-SSHI [61] & Self-Powered Synchronized Switch Harvesting on Inductor \\
\hline SSHO [62] & Synchronized Switch Harvesting on Oscillator \\
\hline SSHI [26] & Synchronized Switch Harvesting on Inductor \\
\hline SSHI-SAMS [63] & $\begin{array}{l}\text { Synchronized Switch Harvesting on Inductor with Self-Adaptive Mechanical } \\
\text { Switches }\end{array}$ \\
\hline SSH-t [64] & Synchronized Switch Harvesting on Inductor with threshold detection \\
\hline $\mathrm{S}^{3} \mathrm{HI}[57]$ & Surge-inducing Synchronized-Switch Harvesting on Inductor \\
\hline \multicolumn{2}{|r|}{ Load adaptation and derivatives } \\
\hline \multicolumn{2}{|r|}{ Architecture acronyms } \\
\hline LLA $[48]$ & Linear Load Adaptation (identical to SEH) \\
\hline SEH interface $[65,66,40]$ & Standard Energy Harvesting interface \\
\hline MPPT-DC [49] & Maximum Power Point Tracking on DC load (identical to SEH) \\
\hline \multicolumn{2}{|r|}{ Acronyms for techniques } \\
\hline FB & Full-Bridge \\
\hline $\mathrm{HB}$ & Half-Bridge \\
\hline
\end{tabular}

In section 2, we start by an analysis to explain the hierarchy proposed in Figure 1. In section 3, we summarize the full mathematical background that is required to develop efficient MPPT strategies, following a similar approach as Halvorsen et al. [8] or Hosseinloo et al. [9]. After providing a mathematical demonstration of the maximum power that an electromechanical resonator is able to deliver, we develop a general expression of the condition to achieve the maximum power point. In section 4 , we adapt this condition to existing harvesting techniques and compare the voltage waveforms, as well as the optimal control parameters. A summary of the main results is given in section 5 . 
2. Existing architectures for single-parameter maximum power point tracking

\subsection{1-stage topology}

The topology that involves the smallest number of stages is a 1-stage (or single-stage) topology. The only 1-stage topology developed in the literature is the OSECE architecture [40] (or its tunable equivalent SECPE [41]). In this architecture, a single charge extraction stage is used. Even though diodes are under use in the proposed implementations, they do not perform the function of a rectifier, which is why it is considered as a 1-stage topology. All other existing architectures request at least one other function, as we will see in the next subsections.

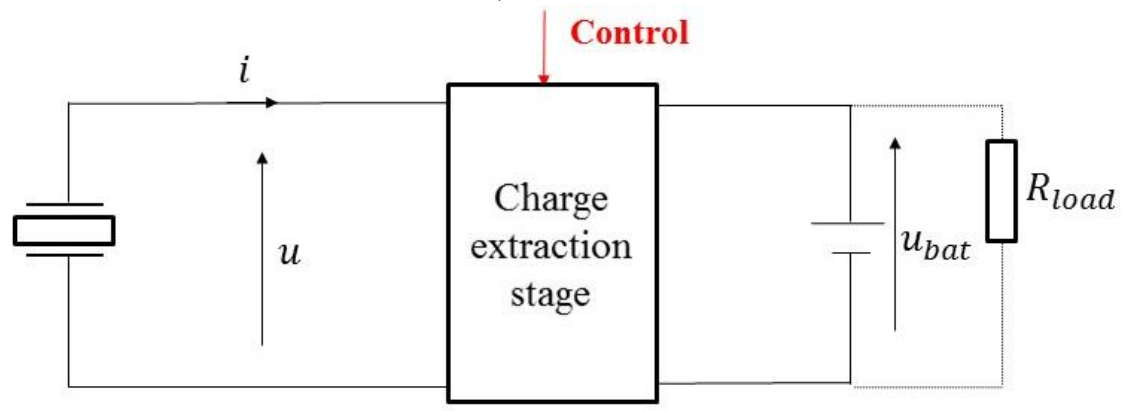

Figure 2: Single 1-stage topology for piezoelectric energy harvesting developed in previous literature.

\subsection{2-stage topologies}

One of the most standard circuits for PEH is made of a rectifier directly plugged to a DC-DC converter structure [67]. The resulting topology is depicted in Figure 3. This structure will be referred to as the 2-stage load adaptation topology. The main idea is that the DC-DC converter stage is equivalent to a resistive load, tunable by adjusting the control signal imposed at the DC-DC converter stage (see Figure 3)

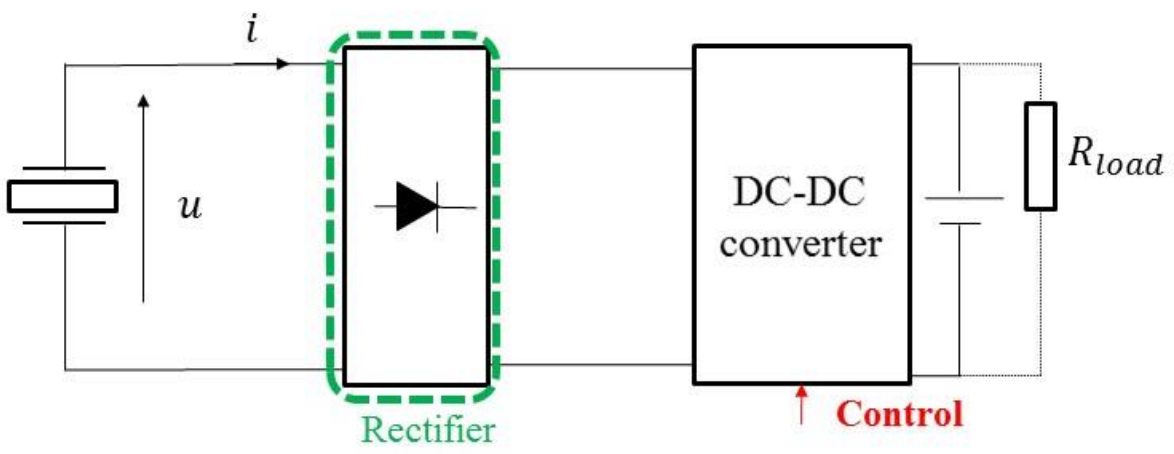

Figure 3: Example of 2-stage topology: 2-stage load adaptation topology

Another 2-stage topology exists, where the two stages are respectively a rectifier stage and an electric charge extraction stage. Even though the practical implementation (in terms of electronic components) may be similar to some 2-stage load adaptation circuits, the function of the subsystem placed between the rectifier and the load is significantly different. This results in a better independence to $R_{\text {load }}$ and a very different MPPT strategy. Moreover, the SECE architecture is sometimes inverted, the charge extraction stage being put before the rectifier (see Figure 4). Such a modification is not possible in the load adaptation architecture. Amongst the architectures which enter is the category of 2-stage topology, one may find in the literature: SECE (either classical, tunable $[51,24]$ and/or unipolar or PSSECE) or rectifier-free SECE (either classical or tunable) [68,69], as previously illustrated in Figure 1. 

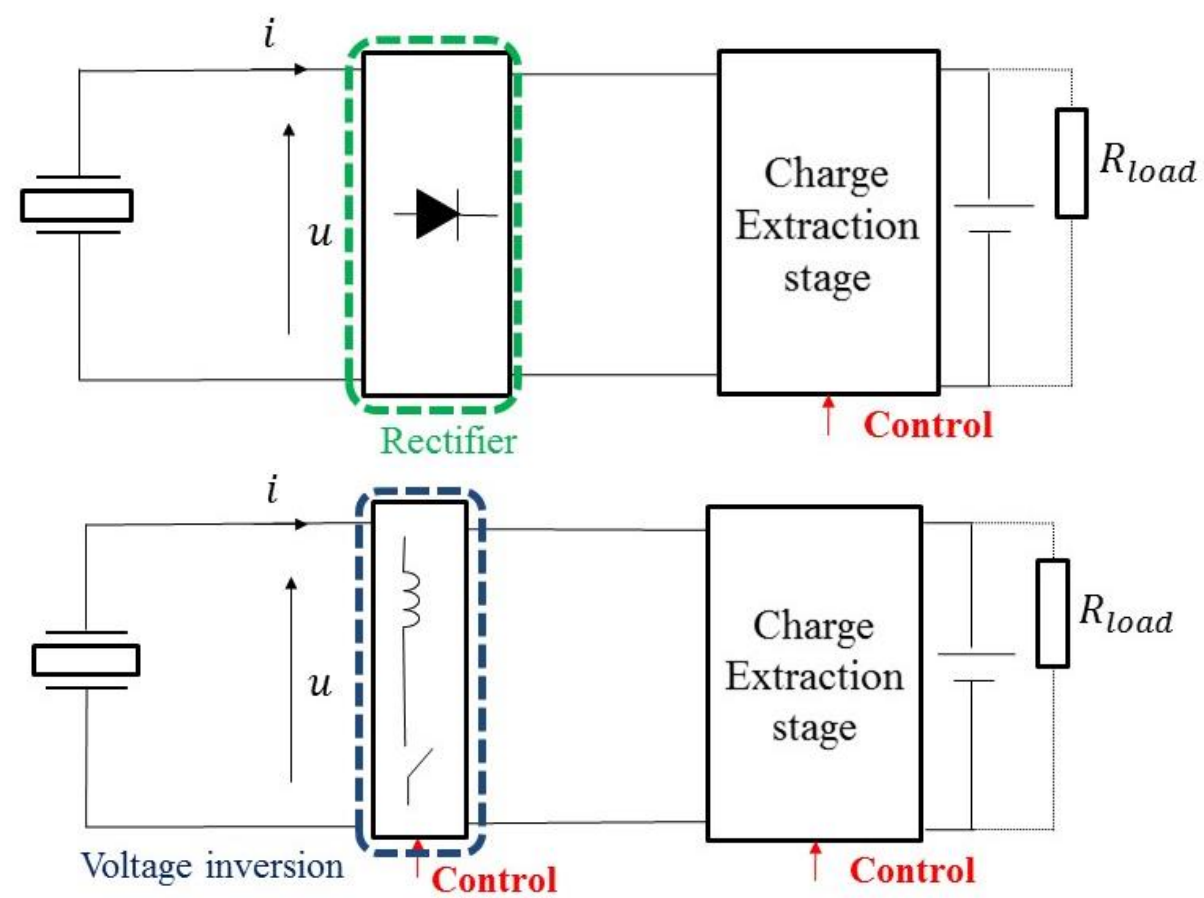

Figure 4: Example of 2-stage topologies: SECE (up) and rectifier-free SECE topologies (down). In some cases, the order may be reversed between the rectifier stage and the charge extraction stage (see FTSECE in section 4.2.2.4).

\subsection{3-stage topologies}

Several 3-stage topologies exist for piezoelectric energy harvesting implementation. The most classical way to implement a 3-stage topology is to add a smoothing stage, usually simply made of a smoothing capacitor. The result is depicted in Figure 5. One of the 3-stage topologies, based on a FB rectifier, has been studied in plenty articles, under various denominations : ACDC power harvesting circuit [70], Standard Energy Harvesting (SEH) interface [65, 66, 40], Linear Load Adaptation (LLA) [48] or even MPPT-DC [49].
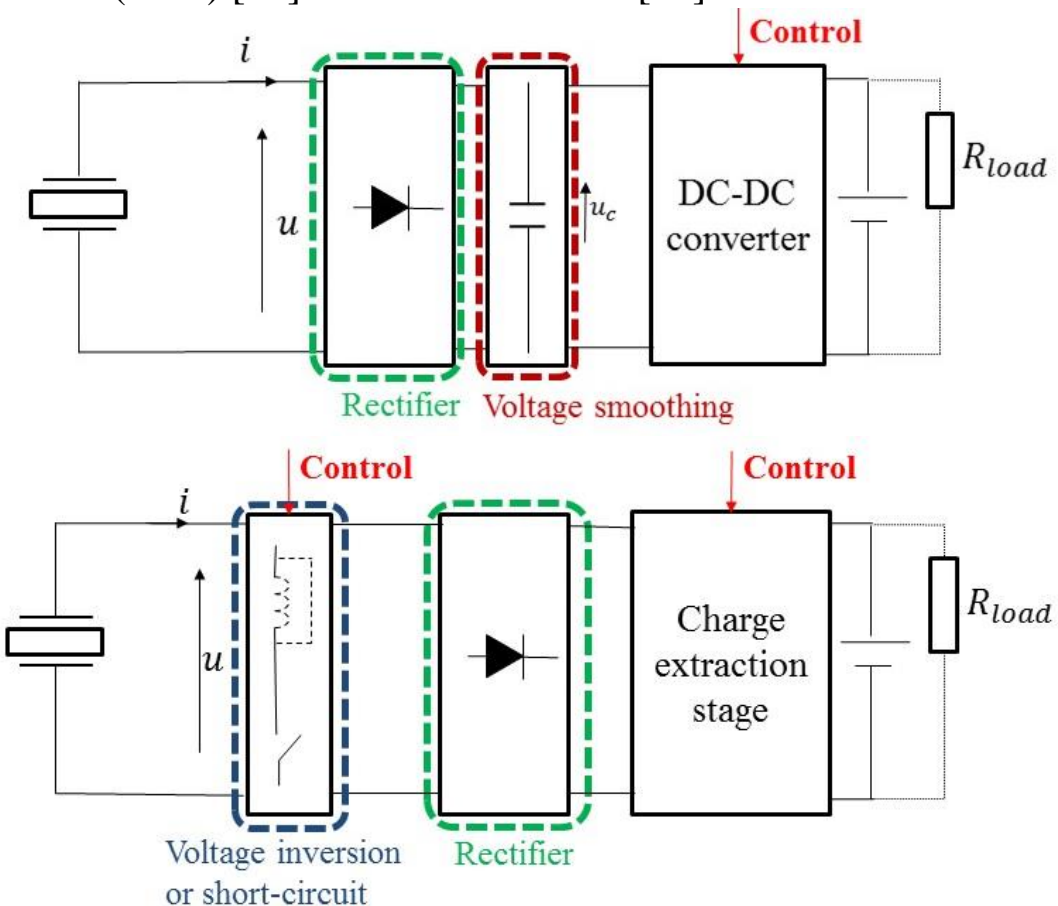

Figure 5: Examples 3-stage topologies: 3-stage load adaptation topology (up) and hybrid-SECE topology (SICE or SCSECE, down). 
Interestingly, another proposition of 3-stage topology has been published by Lallart et al. [52] and makes use of a voltage inversion stage, a rectifier stage and a synchronized charge extraction stage (see Figure 5). It was called SICE (for Synchronous inversion and charge extraction). Finally yet importantly, another architecture enters the category of 3-stage topology [53]. It is called SC-SECE (Short-Circuit Synchronized Electric Charge Extraction) and is based on the addition of a parallel switch to a standard SECE architecture. It behaves slightly differently from SICE but the two structures are extremely similar. Since they are based on the SECE architecture with an additional stage, we have grouped them under the denomination "hybrid SECE".

\subsection{4-stage topology}

The last category of architectures which allow intelligent control strategies can be grouped under the name "4-stage topologies" since they involve 4 stages, each one having a specific function. All the existing architectures made of 4 stages have been called "SSH" (Synchronized Switching Harvesting) and involve a voltage-inversion stage (either with an inductor (SSHI) or with a succession of active switches (SSHC [32] for "synchronized switch harvesting on capacitors")), a rectifier, a voltage smoothing stage and a DC-DC converter. The general topology of all SSH circuits including ESSH [55] (for "enhanced synchronized switch harvesting"), DSSH [54] (for "double synchronized switch harvesting"), p-SSHI [71], s-SSHI [72], multiple-step SSHI [73, 74] or SSHC [32] is reported in Figure 6.

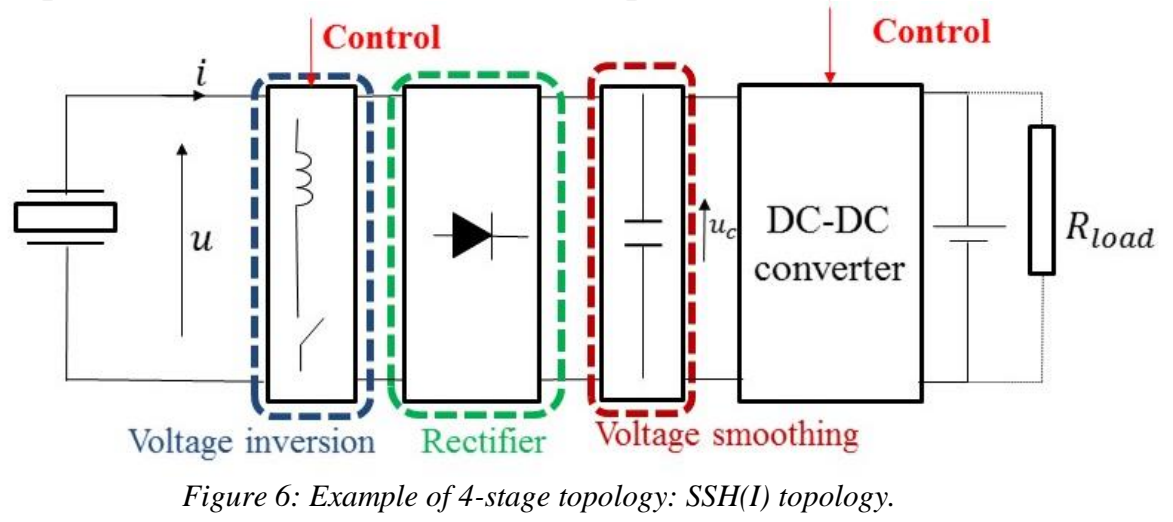

Now that we have reminded and categorized the existing architectures which allow MPPT strategies, we will determine the conditions for which each of them brings out the maximum available power.

\subsection{Remark on power management systems}

One should keep in mind that we focus here on the primary interface, which does not guarantee a constant output voltage, usually required to supply electronic systems. To make a full system able to power systems such as sensor or sensor nodes, one must usually add a "secondary interface" called "power management circuit" at the output of all the aforementioned topologies, whose function is to provide the desired fixed output voltage. Between the primary and the secondary interface, energy storage (for example a battery or supercapacitor) is needed to avoid a conflict between MPPT (realized by the primary interface) and voltage regulation (managed by the secondary interface), as depicted in Figure 7. Moreover, the power management system must sometimes include a dedicated cold-startup to minimize the time to reach steady state [75]. In this study, we only focus on the steady-state configuration, independently of the transient regime at start-up. 


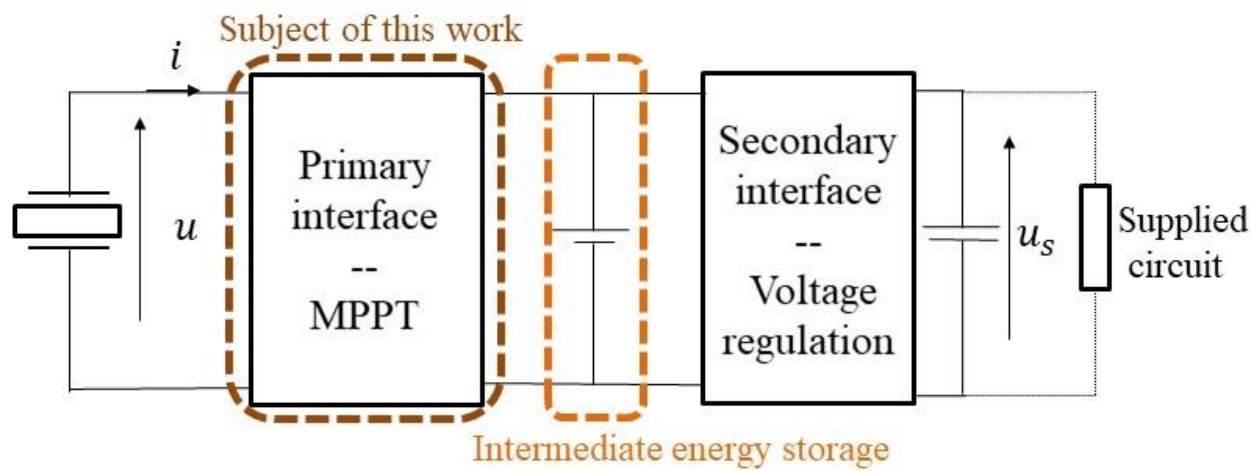

Figure 7: Full system including the primary interface for MPPT, the intermediate energy storage, the secondary interface for voltage regulation and the supplied circuit.

3. Electromechanical model of a piezoelectric transducer

\subsection{General solution}

To provide a review about existing methods, a general theory of optimal control of MPPT systems must be developed. To understand the behavior of piezoelectric energy harvesters, one must first understand the behavior of piezoelectric transducers. Linear inertial piezoelectric energy harvesters are usually composed of a layer of piezoelectric transducer beam stuck on a mechanical resonator (e.g. a cantilever). In this study, we focus on the impact of nonlinear conditioning circuits on energy harvesters powered by a linear SDOF (single-degree-offreedom) piezoelectric resonator. Even though the assumption of linearity is sometimes debatable [76, 77], it remains a reasonable and common basis to compare the performance of electronic circuits [78] [65]. Some problems have been highlighted with this model and, in some cases (for example if there is no mass added at the tip of the beam or if the added mass is small compared to the mass of the beam), the model can be adapted with correction factors [79] [55]. Here, we assume that the transducer has only a single beam mode with one pair of electrodes. For such a resonator, the SDOF lumped model depicted in Figure 8 provides a reliable representation around one of its resonant frequencies [79]. It is composed of an inertial mass $M$ suspended by a linear spring of linear stiffness $K$ and nonlinear stiffness $K_{2}(x)$. The damper $c$ models the mechanical losses of the system. External displacement $y$ (acceleration $\gamma(t)$ ) results in a mechanical displacement of the resonator base. We suppose a harmonic vibration source and write $\gamma_{m}$ the amplitude of $\frac{d^{2} y}{d t^{2}}=\gamma_{m} \sin \omega t$. The vibration gives rise to a relative displacement $x$ of the inertial mass with respect to the base. The bidirectional electromechanical coupling is achieved by the piezoelectric material through a piezoelectric coupling term $\alpha$.

The normalized variables and normalization factors are reported in Table 2. In the following sections, all the voltages will be normalized with respect to $M \gamma_{m} / \alpha$ as for the piezoelectric voltage $u$ (normalized into $U$ ). Capital letters will refer to normalized quantities, whereas small variable letters will refer to the physical (non-normalized) quantities. This normalization has already been proposed in previous works like [51] and makes the mathematical treatment of the problem much simpler, as the reader will notice in this section. 


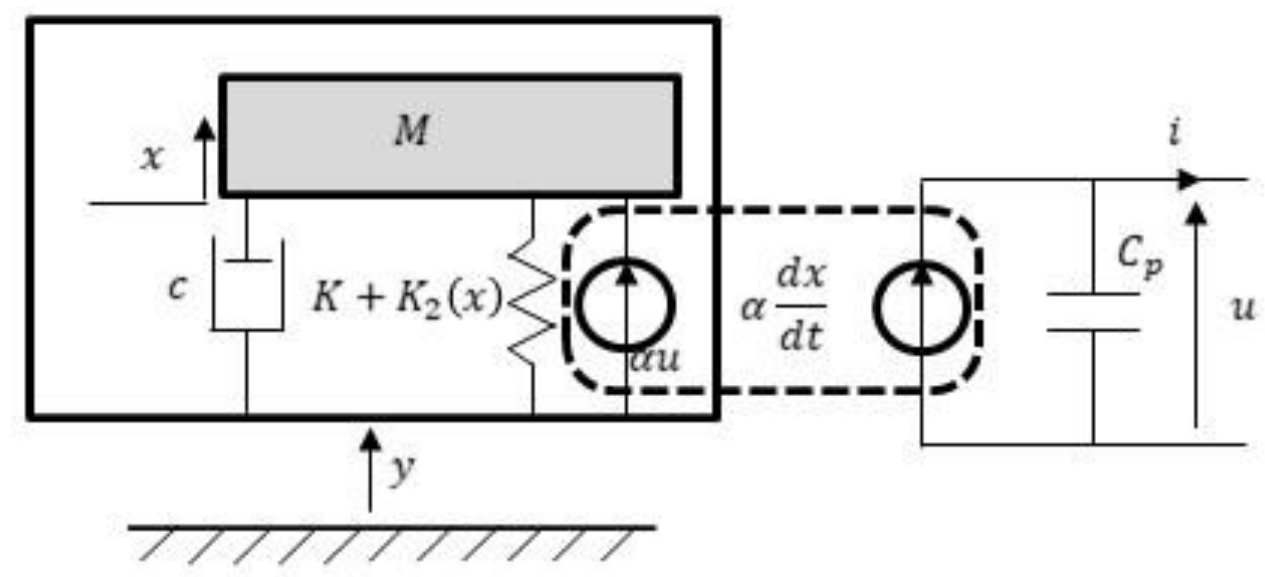

Figure 8: SDOF model of inertial piezoelectric energy harvesters.

TABLE 2. VARIABLES AND NORMALIZATIONS

\begin{tabular}{ccc}
\hline \hline Variable & Quantity (unit) & Normalized variable \\
\hline$\omega$ & Vibration angular frequency (rad. ${ }^{-1}$ ) & $\Omega=\frac{\omega}{\omega_{0}}$ \\
$y$ & Displacement of the base (m) & $Y=\frac{y \omega_{0}^{2}}{\gamma_{m}}$ \\
$x$ & Displacement of the resonator (m) & $X=\frac{x \omega_{0}^{2}}{\gamma_{m}}$ \\
$i$ & Piezoelectric current (A) & $I=\frac{i \alpha}{C_{p} M \omega_{0} \gamma_{m}}$ \\
\hline \hline
\end{tabular}

In this article, as in most previous works, we choose to neglect the dielectric losses in the piezoelectric layer [24]. This is a reasonable assumption for hard piezoelectric ceramics, which tend to exhibit a large dielectric permittivity, but may fall short for "lossy" materials such as piezoelectric monocrystals or soft ceramics [80]. Under all the aforementioned assumptions, the governing equations of the SDOF system are (1).

$$
\left\{\begin{array}{c}
M \frac{d^{2} x}{d t^{2}}+c \frac{d x}{d t}+K x+K_{2}(x) x+\alpha u=-M \frac{d^{2} y}{d t^{2}} . \\
i=\alpha \frac{d x}{d t}-C_{p} \frac{d u}{d t}
\end{array} .\right.
$$

Based on the parametrization of Table 2, (1) becomes (2),

$$
\left\{\begin{array}{c}
\ddot{X}+\frac{1}{Q} \dot{X}+X+\frac{K_{2}(X)}{K} X+U=-\ddot{Y} \\
I=k_{m}^{2} \dot{X}-\dot{U}
\end{array}\right.
$$

where the "dotted" notation relates to the derivative with respect to the angle $\theta=\omega_{0} t$ and where $k_{m}^{2}=\alpha^{2} / K C_{p}$ is the expedient electromechanical coupling coefficient. In some case, it 
is also useful to define the electromechanical coupling coefficient as $k^{2}=\frac{\alpha^{2}}{K C_{p}+\alpha^{2}}$. With this definition, $0 \leq k^{2}<1$ whereas $k_{m}^{2}$ may take any positive value.

The mean power extracted from the generator is (3)

$$
p=\frac{C_{p} M K \gamma_{m}^{2}}{\alpha^{2} \omega_{0}} \frac{\Omega}{2 \pi} \int_{0}^{\frac{2 \pi}{\Omega}} U(\theta) I(\theta) d \theta
$$

which leads to (4) if the piezoelectric voltage is $2 \pi / \Omega$-periodic.

$$
\begin{gathered}
p=-\frac{C_{p} M K \gamma_{m}^{2}}{\alpha^{2} \omega_{0}} \frac{\Omega}{2 \pi} \int_{0}^{2 \pi / \Omega}\left[\ddot{X}(\theta)+\frac{1}{Q} \dot{X}(\theta)+X(\theta)+\frac{K_{2}(X(\theta))}{K} X(\theta)\right. \\
+\ddot{Y}(\theta)] k_{m}^{2} \dot{X} d \theta
\end{gathered}
$$

Finally, one obtains (5) as long as $X$ and $\dot{X}$ are $2 \pi / \Omega$-periodic.

$$
p=-\frac{M \gamma_{m}^{2}}{\omega_{0}} \frac{\Omega}{2 \pi} \int_{0}^{2 \pi / \Omega}\left[\frac{1}{Q} \dot{X}(\theta)^{2}+\ddot{Y}(\theta) \dot{X}(\theta)\right] d \theta
$$

Writing $X=a_{X_{0}}+\sum_{n=1}^{\infty}\left[a_{X_{n}} \cos (n \Omega t)+b_{X_{n}} \sin (n \Omega t)\right]$, the derivative of $X$ is (6).

$$
\dot{X}=\Omega \sum_{n=1}^{\infty} n\left[b_{X_{n}} \cos (n \Omega \theta)-a_{X_{n}} \sin (n \Omega \theta)\right]
$$

The Parseval equality combined with the assumption of a sine-wave acceleration $\ddot{Y}=\sin (\Omega t)$ leads to (7).

$$
p=\frac{M \gamma_{m}^{2}}{\omega_{0}} \frac{\Omega}{2}\left(a_{X_{1}}-\frac{\Omega}{Q}\left[\sum_{n=1}^{\infty} n^{2}\left(a_{X_{n}}^{2}+b_{X_{n}}^{2}\right)\right]\right)
$$

Equation (7) shows that, if the motion of the piezoelectric transducer exhibits upper harmonics (for example due to a harvesting strategy that generates upper harmonics in the piezoelectric voltage), all the energy contained in these upper harmonics is necessarily lost (dissipated) as long as the vibrations of the environment remain sinusoidal. In itself, this interesting result remains true even for a resonator with a nonlinear stiffness.

Now, for a given frequency $\Omega$, we are looking for the maximum power which can be extracted from the piezoelectric generator. Based on (7), one may find the upper bound (8).

$$
p \leq \frac{M \gamma_{m}^{2}}{\omega_{0}} \frac{\Omega}{2}\left(a_{X_{1}}-\frac{\Omega}{Q} a_{X_{1}}^{2}\right)
$$

This upper bound is achieved when (9) is verified. 


$$
\left\{\begin{array}{c}
a_{X_{1}}=\frac{Q}{2 \Omega} \\
\forall n>1, b_{X_{1}}=a_{X_{n}}=b_{X_{n}}=0 \\
p=p_{\text {lim }}=\frac{M \gamma_{m}^{2} Q}{8 \omega_{0}}
\end{array}\right.
$$

Interestingly, (9) also holds for any nonlinear mechanical stiffness term $K_{2}(X)$. The optimal trajectory is a sine wave, in quadrature with the motion $Y$ of the base. This result is a particular (but useful) example of a more general result demonstrated in [9]. In this case, the mean power dissipated, per period, in the mechanical damped is then:

$$
p_{\text {diss }}=\frac{\gamma_{m}^{2}}{\omega_{0}^{4}} \frac{M \omega_{0}}{Q} \omega_{0}^{2} \frac{\Omega}{2 \pi} \int_{0}^{\frac{2 \pi}{\Omega}} \dot{X}^{2} d \theta=\frac{M \gamma_{m}^{2} Q}{8 \omega_{0}}
$$

The corresponding electromechanical conversion efficiency (i.e. the ratio between the mean extracted power $p$ and the mean available mechanical power) is thus only $50 \%$ when the maximal power is extracted:

$$
\eta=\frac{p}{p+p_{\text {diss }}}=50 \%
$$

This is a well-known result in specific cases (like a linear resonator), already demonstrated for an arbitrary nonlinear stiffness [81]. When reaching an electromechanical conversion efficiency $\eta$ superior to $50 \%$, one can be sure that the maximum power point is not achieved. This demonstration also proves that, in the scenario where the base acceleration is sinusoidal and where the maximum available power is reached, all the energy that would lie in the upper harmonics of the motion of the piezoelectric generator cannot be harvested. However, one must not be confused: this demonstration does not prove that the energy lying in the upper harmonics of the motion can never be harvested. It only proves that, with a sine-wave acceleration, one cannot hope to take advantage of the mechanical energy lying in the upper harmonics to reach the maximum power that such a resonator can deliver. This statement does not apply with random vibrations and/or with multimodal resonators [82].

The verification that the maximum power predicted by simulations does not surpass the theoretical bound should be made every time a new control scheme is studied. Some errors can be found is existing literature, telling that more power than $p_{\text {lim }}$ can be achieved (for example for so-called energy injection architectures [7] [83] [84]).

\subsection{Case of a mechanically-linear resonator}

For a linear resonator $\left(K_{2}(X)=0\right)$, we reach an interesting conclusion by checking the corresponding voltage. Based on a Taylor-Series expansion of the piezoelectric voltage $U(\theta)=a_{U_{0}+} \sum_{n=1}^{\infty}\left[a_{U_{n}} \cos (n \Omega \theta)+b_{U_{n}} \sin (n \Omega \theta)\right]$, the condition (9) for MPPT brings out (12) and (13).

$$
\forall n \neq 1,\left\{\begin{array}{l}
a_{X_{n}}\left(1-n^{2} \Omega^{2}\right)+\frac{b_{X_{n}} n \Omega}{Q}=-a_{U_{n}} \\
b_{X_{n}}\left(1-n^{2} \Omega^{2}\right)-\frac{a_{X_{n}} n \Omega}{Q}=-b_{U_{n}}
\end{array}\right.
$$


and

$$
\left\{\begin{array}{c}
a_{X_{1}}\left(1-\Omega^{2}\right)+\frac{b_{X_{1}} \Omega}{Q}=-a_{U_{1}} \\
b_{X_{1}}\left(1-\Omega^{2}\right)-\frac{a_{X_{1}} \Omega}{Q}=-b_{U_{1}}-1
\end{array}\right.
$$

Equivalently to (9), we can also express the condition to achieve the maximum power $p_{\text {lim }}$ as (14) :

$$
\left\{\begin{array}{c}
a_{U_{1}}=\frac{Q}{2 \Omega}\left(\Omega^{2}-1\right) \\
b_{U_{1}}=-\frac{1}{2} \\
\forall n>1, a_{U_{n}}=b_{U_{n}}=0
\end{array}\right.
$$

This last expression (14) is extremely useful for practical implementation, because it means that, providing that the system is able to reach the maximum power $P_{\text {lim }}$, a real-time estimation of the first-harmonic voltage components $a_{U_{1}}$ and $b_{U_{1}}$ allows real-time optimization of the extracted power without actually measuring the power.

\subsection{Simplification}

If one writes the transfer function between the first-harmonic voltage and the first-harmonic motion $\frac{U_{1}}{\underline{X_{1}}}=k_{m}^{2}\left(U_{p}(\Omega)+j U_{q}(\Omega)\right)$, the combination of (9) and (13) leads to condition (15) to ensure that the maximum power $P_{\text {lim }}$ is extracted from the generator.

$$
\left\{\begin{array}{c}
\Omega^{2}=1+k_{m}^{2} U_{p}(\Omega) \\
k_{m}^{2} Q=-\frac{\Omega}{U_{q}(\Omega)}
\end{array}\right.
$$

If we focus on single-parameter MPPT, one can write :

$$
\left\{\begin{array}{c}
\Omega^{2}=1+k_{m}^{2} U_{p}(\Omega, v(\Omega)) \\
k_{m}^{2} Q=-\frac{\Omega}{U_{q}(\Omega, v(\Omega))}
\end{array}\right.
$$

where $v$ is the tuning parameter. In some specific cases $U_{p}$ is constant and only one frequency yields the maximum power: $\Omega=\sqrt{1+k_{m}^{2} U_{p}}$.

The second equation of (15) justifies why $k_{m}^{2} Q$ is usually called a figure-of-merit (FOM). It defines a necessary condition for the maximum power to be reachable, as we will illustrate in the following developments.

\subsection{Non-adaptive MPPT control and examples}

If we consider the simple case where no optimization parameter is available, one may deduce, from (15), that the maximum power may only be achieved when $\Omega^{2}=1+k_{m}^{2} U_{p}(\Omega)$ has at least one solution and when $k_{m}^{2} Q=-\frac{\Omega}{U_{q}(\Omega)}$ for the corresponding frequency. As long as $U_{p}(\Omega)$ and $U_{Q}(\Omega)$ are fractions of polynomials in $\Omega$, only a finite number of $k_{m}^{2} Q$ values allow to achieve the maximum available power, each one at a finite number of specific corresponding 
frequencies. The most simple example of such a statement correspond to constant in-phase and quadrature components $U_{p}$ and $U_{q}$. For instance, classical SECE, where $\underline{U_{1}}=k_{m}^{2} \underline{X_{1}}\left(1-j \frac{4}{\pi}\right)$, achieves the maximum power point only when (17) is satisfied.

$$
\left\{\begin{array}{c}
\Omega^{2}=1+k_{m}^{2} \\
k_{m}^{2} Q=\frac{\pi \sqrt{1+k_{m}^{2}}}{4}
\end{array}\right.
$$

We obtain the condition $k_{m}^{2} Q=\frac{\pi\left(\pi+\sqrt{\pi^{2}+64 Q^{2}}\right)}{32 Q}$ which, for high quality factors, simplifies into $k_{m}^{2} Q=\pi / 4$ which is a well-known result [21].

Now that we have developed the basic knowledge required to understand the control design rules in order to extract the maximum power, section 4 illustrates these rules on existing energy harvesting techniques.

4. Classification and characteristics of existing adaptive control strategies

4.1.1-stage topology : the tunable OSECE technique (SECPE)

As stated in section 2.1, the single existing architecture with 1-stage topology is the OSECE architecture. Recent publications focus on compact autonomous OSECE circuits, like the (C)SPOSECE (for "(Compact) Self-Powered Optimized Synchronized Electric Charge Extraction") [44]. The classical OSECE technique does not reach the maximum available power except for one specific value of the FOM $k_{m}^{2} Q$ (see remark 3.4). In its tunable version, called SECPE by its authors [41], the duration of charge extraction is adjusted to maximize the power flow. From the piezoelectric perspective, this case is identical to tunable SECE [24]. The corresponding waveforms are depicted in Figure 9. In this case, $U_{p}(\Omega)=1$ and $U_{q}(\beta(\Omega))=$ $-\frac{4}{\pi} \frac{1-\beta}{1+\beta}$ (with $\left.\beta \in\right]-1,1[$ the tuning factor corresponding to the voltage drop during charge extraction, see Figure 9). 

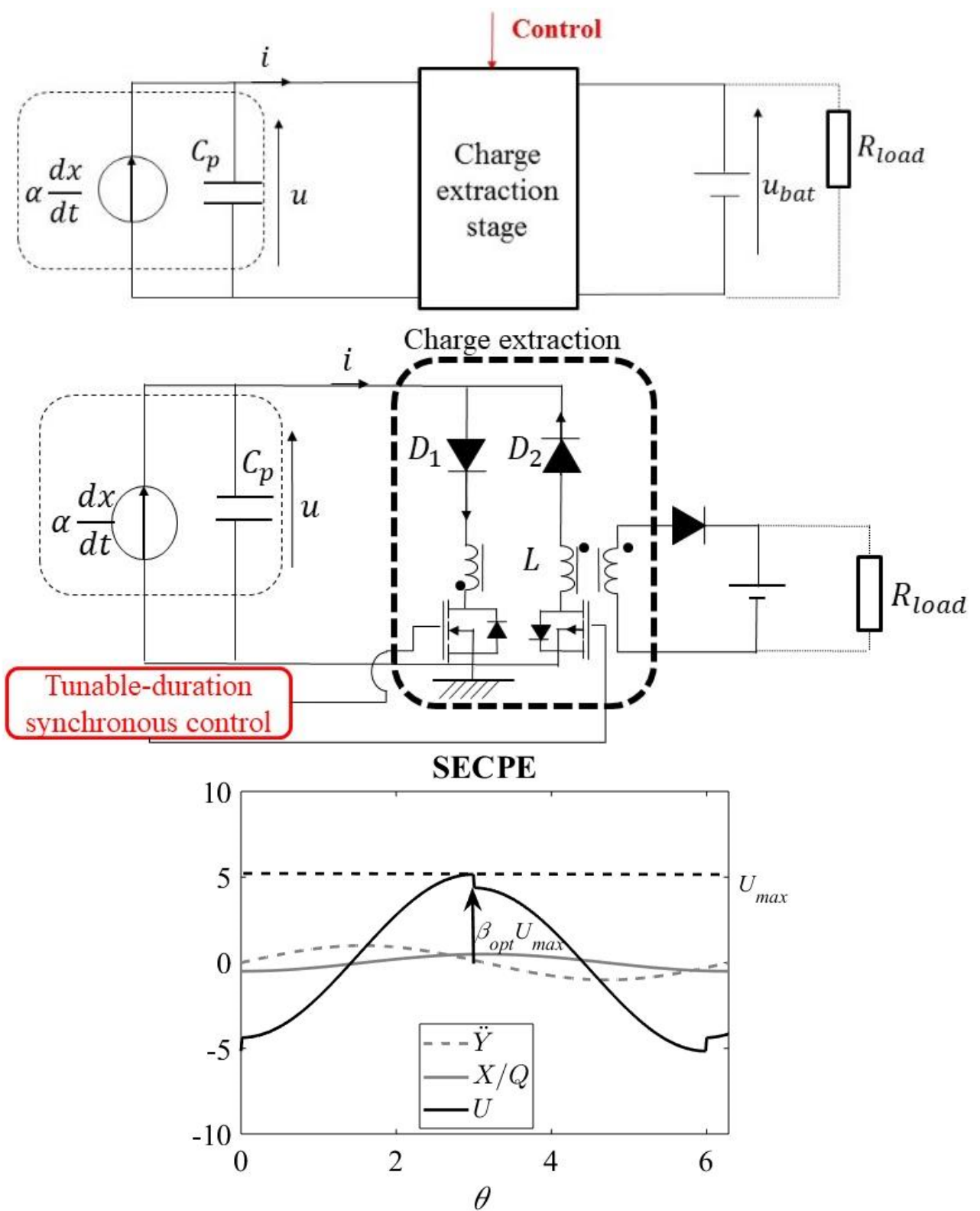

Figure 9: Topology (top), architecture (middle) and waveforms at the maximum power point (bottom) in the SECPE technique at the optimal power point $\Omega=\Omega_{\text {opt }}=\sqrt{1+k_{m}^{2}}, \beta=\beta_{\text {opt }}, k_{m}^{2}=0.1, Q=100$.

Hence, from (15), the optimal power is obtained when (18) is verified.

$$
\left\{\begin{array}{c}
\Omega_{o p t}^{2}=1+k_{m}^{2} \\
k_{m}^{2} Q=\frac{\Omega}{\frac{4}{\pi} \frac{1-\beta_{o p t}}{1+\beta_{o p t}}}
\end{array}\right.
$$

This gives (19) as the optimal operating point.

$$
\left\{\begin{array}{c}
\Omega=\Omega_{o p t}=\sqrt{1+k_{m}^{2}} \\
\beta=\beta_{o p t}=\frac{4 k_{m}^{2} Q-\pi \sqrt{1+k_{m}^{2}}}{4 k_{m}^{2} Q+\pi \sqrt{1+k_{m}^{2}}}
\end{array}\right.
$$

Hence, in the case of tunable SECE, the maximum power can be achieved at one single frequency $\Omega=\sqrt{1+k_{m}^{2}}$, under no constraint on $k_{m}^{2} Q$. 


\subsection{2-stage topologies}

4.2.1. 2-stage load adaptation architecture

\subsubsection{2-stage load adaptation FB technique}

Among the existing topologies, a 2-stage load adaptation topology called "buck-boost topology without input filtering capacitor" $[85,67,86]$ has been proposed. It is based on the use of rectifier architecture (either FB [87] or shunt [37]) directly followed a DC-DC converter whose switching frequency is significantly larger than the frequency of the piezoelectric vibration. If the buck-boost converter is in discontinuous conduction mode, its equivalent impedance is independent of the load $R_{\text {load }}$ placed at its output. In such case, the DC-DC converter behaves as a resistive load $R_{e q}$ (see (20)) which depends only on the characteristics of the DC-DC converter (switching frequency $f_{S}$, duty-cycle $\delta$ and inductance $L$ ) (20).

$$
R_{e q}=\frac{2 L f_{s}}{\delta^{2}}
$$

Under these assumptions, we will consider the DC-DC converter as a single-parameter tunable stage with one degree of freedom $R_{e q}$ for the optimization process. In the following developments, we will normalize $R_{e q}$ into the normalized resistance $r=R_{e q} C_{p} \omega_{0}$.

For a FB rectifier, the piezoelectric voltage waveform is a sine wave depicted in Figure 10. From the piezoelectric perspective, this solution is equivalent to plugging directly a resistor $R_{e q}$. 

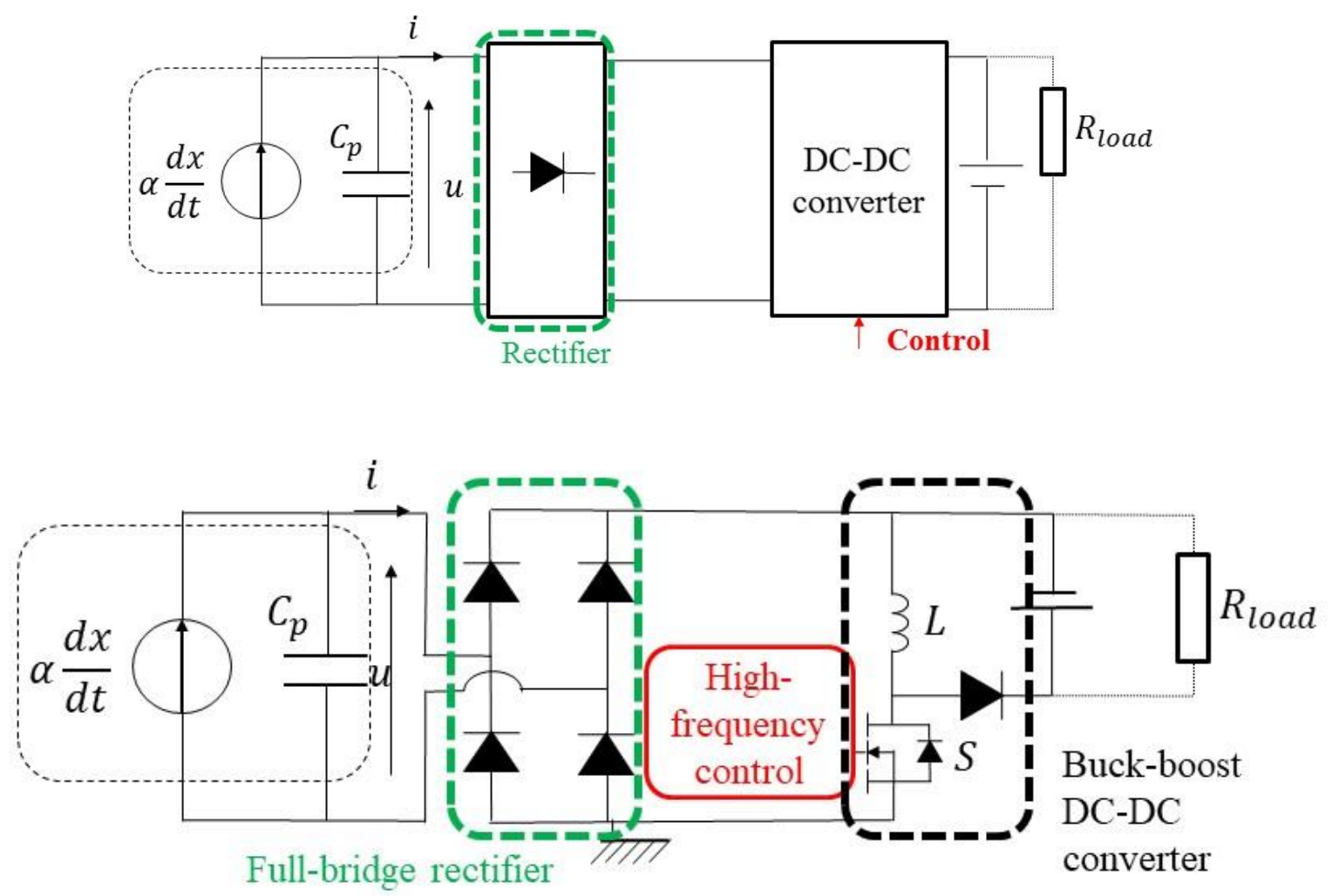

2-stage FB load adaptation
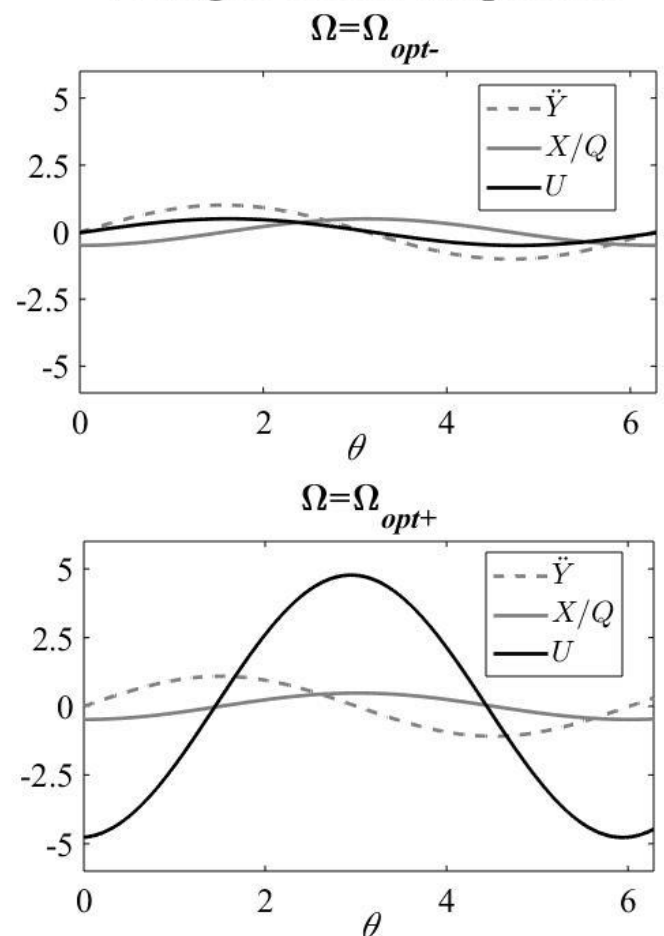

Figure 10: Topology (top), architecture (middle) and waveforms at the maximum power point (bottom) in the 2-stage load adaptation FB technique. Example for $k_{m}^{2}=0.1, \Omega=\Omega_{o p t \pm}, r\left(\Omega_{o p t \pm}\right)=r_{o p t \pm}$ and $Q=100$.

The electrical behavior is described by (21) which gives the in-phase and quadrature components of the voltage $U(22)$.

$$
\frac{U}{r}=k_{m}^{2} \frac{d X}{d \theta}-\frac{d U}{d t}
$$




$$
\left\{\begin{array}{c}
U_{p}(\Omega, r(\Omega))=\frac{r^{2} \Omega^{2}}{1+r^{2} \Omega^{2}} \\
U_{q}(\Omega, r(\Omega))=-\frac{\Omega r}{1+r^{2} \Omega^{2}}
\end{array}\right.
$$

Hence, the optimality condition (15) becomes (23).

$$
\left\{\begin{array}{c}
\Omega_{o p t}^{2}=1+k_{m}^{2}\left(\frac{r_{o p t}^{2} \Omega_{o p t}^{2}}{1+r_{o p t}^{2} \Omega_{o p t}^{2}}\right) \\
k_{m}^{2} Q=\frac{1+r_{o p t}^{2} \Omega_{o p t}^{2}}{r}
\end{array}\right.
$$

One can conclude from (23) that the maximum power is achievable only if $k_{m}^{2} Q \geq 2\left(\frac{1}{2 Q}+1\right)$ (condition also given in [88]) and that the corresponding frequencies and optimal load are (24).

$$
\left\{\begin{array}{c}
\Omega=\Omega_{o p t \pm}=\frac{\sqrt{k_{m}^{2}-\frac{1-2 Q^{2} \mp \sqrt{1-2\left(2+k_{m}^{2}\right) Q^{2}+k_{m}^{4} Q^{4}}}{Q^{2}}}}{\sqrt{2}} \\
r=r_{o p t \pm}=\frac{1+k_{m}^{2} Q^{2} \pm \sqrt{1-2\left(2+k_{m}^{2}\right) Q^{2}+k_{m}^{4} Q^{4}}}{2\left(1+k_{m}^{2}\right) Q}
\end{array}\right.
$$

As soon as $k_{m}^{2} Q \geq 2\left(\frac{1}{2 Q}+1\right)$, the optimal power is achievable for two different frequencies $\Omega$ and two different resistive loads. We find here the exact analytical expressions of the corresponding resistive load and frequencies $\Omega$ to reach $p=p_{\text {lim }}$. Equation (24) is consistent with previous literature [89] since the optimal resistance $r_{\text {lopt }}\left(\Omega_{o p t}\right)$ is written (25) for all frequencies $\Omega$. As expected, we have $r_{l_{o p t}}\left(\Omega_{o p t \pm}\right)=r_{o p t \pm}$.

$$
r_{\text {lopt }}(\Omega)=\frac{1}{\Omega} \frac{\sqrt{\Omega^{2}+Q^{2}\left(1-\Omega^{2}\right)^{2}}}{\sqrt{\Omega^{2}+Q^{2}\left(1+k_{m}^{2}-\Omega^{2}\right)^{2}}}
$$

In most practical cases, the piezoelectric transducer under use is of high quality factor $Q \gg 1$. In such cases, the condition to reach optimality becomes $k_{m}^{2} Q \geq 2$. Hence, (24) can be approximated by (26).

$$
\left\{\begin{array}{r}
\Omega=\Omega_{o p t \pm} \simeq \sqrt{1+\frac{k_{m}^{2}}{2} \pm \frac{k_{m}^{2}}{2}} \\
r=r_{o p t \pm} \simeq \frac{k_{m}^{2} Q \pm 2 \sqrt{k_{m}^{4} Q^{2}-2\left(2+k_{m}^{2}\right)}}{2\left(1+k_{m}^{2}\right)}
\end{array}\right.
$$

4.2.1.1. 2-stage load adaptation shunt rectifier technique 
The case where the FB rectifier is replaced by a shunt-diode rectifier [37] is, though interesting, very complex to treat mathematically so that numerical computations are the only way to draw conclusions. Unfortunately, contrary to the FB technique, no simple and interpretable mathematical expression can be found for the 2-stage load adaptation shunt-rectifier technique [37]. Numerical simulations suggest that the condition to reach the maximum available power is $k_{m}^{2} Q>3.4 \pi / 4$. ( [37] suggested $k^{2} Q>3.4 \pi / 4$ for very high-Q factor) and that the corresponding optimal frequencies are the same as the HB (or FB) 3-stage load adaptation technique (see section 4.3.2), i.e. (27). The topology, architecture and waveforms at maximum power point are illustrated in Figure 11.

$$
\Omega=\Omega_{o p t \pm} \simeq \sqrt{1+\frac{k_{m}^{2}}{2} \pm \frac{\sqrt{k_{m}^{2} Q\left(k_{m}^{2} Q-\sqrt{1+\frac{k_{m}^{2}}{2} \pi}\right)}}{2 Q}}
$$
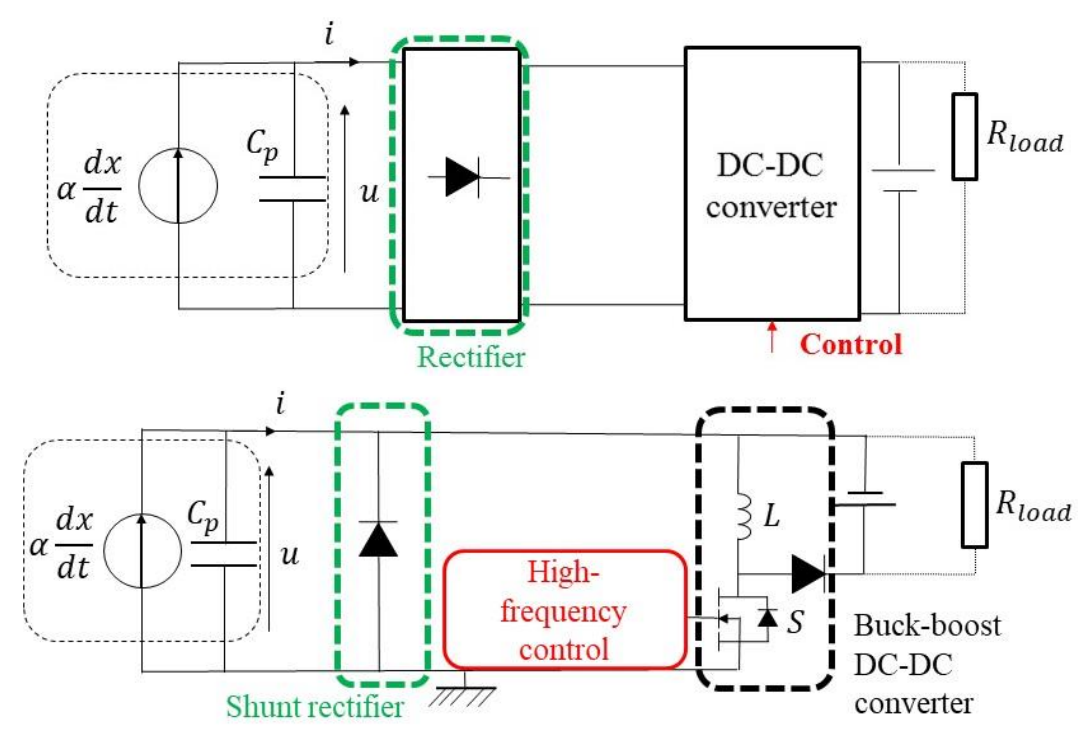

2-stage shunt-rectifier load adaptation
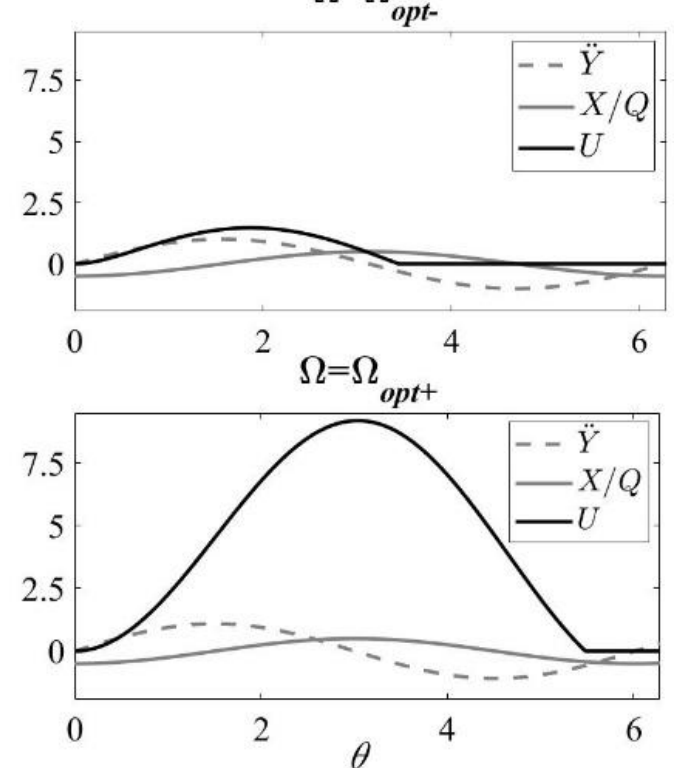

Figure 11: Topology (top), architecture (middle) and waveforms at the maximum power points (bottom) in the 2-stage shunt-rectifier load adaptation technique. Example for $k_{m}^{2}=0.1, \Omega=\Omega_{\text {opt }}, r\left(\Omega_{\text {opt }}\right)=r_{\text {opt } \pm}$ and, $Q=100$. 


\subsubsection{SECE architectures}

As stated in the introduction, SECE architectures (sometimes shortened into SCE [45] [46]) have a 2-stage topology in which the idea is to extract the energy from the generator at very specific instants to maximize the power flow, and to leave the generator in open-circuit most of the time, to avoid electrical damping. Most SECE architectures are based on a full-bridge rectifier and a buck-boost converter structure where the control is at low frequency and synchronous with the oscillation. A variant of this standard architecture also exists with a buck converter structure and was given another name (SSDCI for "Synchronized Switching and Discharging to a storage Capacitor through an Inductor"). Its authors initially stated that it is functionally similar to SECE [90].

Some integrated SECE circuits already exist [35] [48] [91], some of which are dedicated to unsteady vibrations (shocks) [91] or multi-beam generators [92]. Other works have proposed a rectifier-less architecture where they replace the full-bridge rectifier by active switch resulting in a different 2-stage topology [93] [94]. Even with all these existing demonstrations of SECE circuits, MPPT algorithms remain quite a hot topic, which requires a thoughtful analytical study. In this section, we perform this study for existing SECE-based techniques.

\subsubsection{PS SECE technique}

In the case of PS SECE, the rectifier is a full-bridge rectifier and the tuning parameter is the instant of charge extraction. There is no smoothing capacitor. The corresponding voltage waveform is as depicted in Figure 12. 

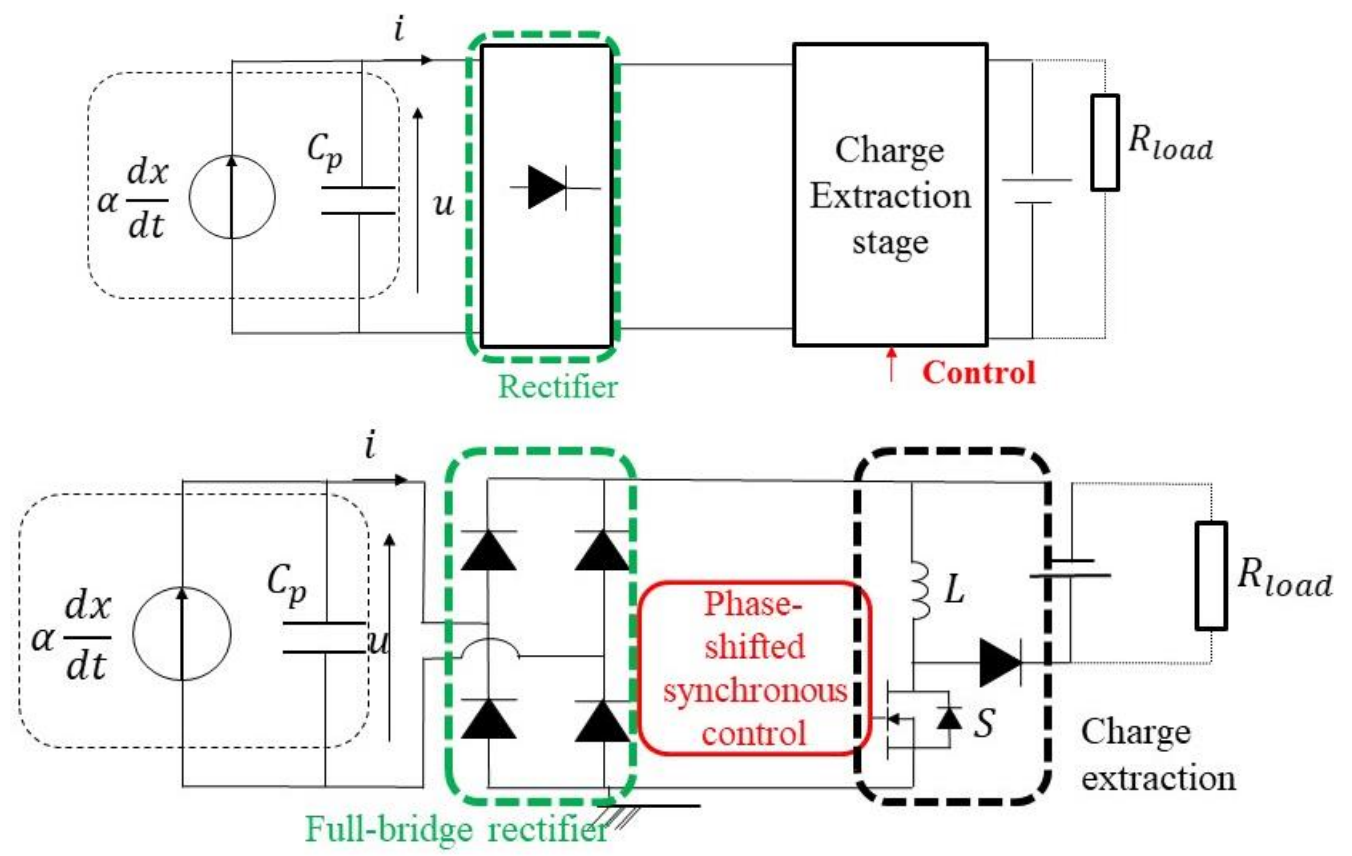

PS SECE
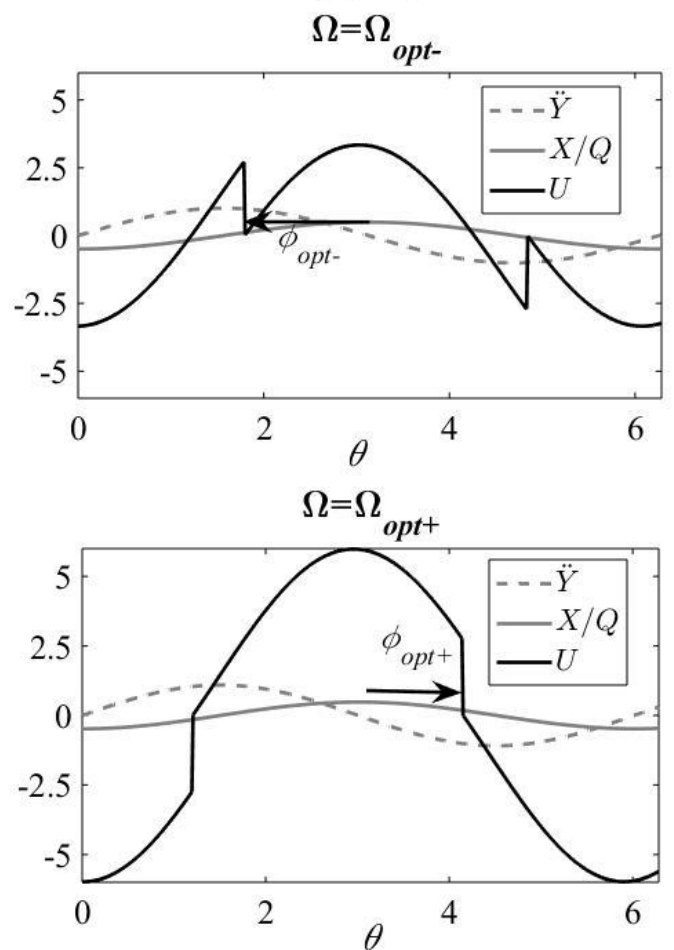

Figure 12: Topology (top), architecture (middle) and waveforms at the maximum power points (bottom) for PS SECE technique. Example for $k_{m}^{2}=0.1, \Omega=\Omega_{\text {opt }}, r\left(\Omega_{\text {opt } \pm}\right)=r_{\text {opt } \pm}$ and, $Q=100$.

Such waveforms lead to $U_{p}(\phi(\Omega))=1+\frac{2}{\pi} \sin 2 \phi$ and $U_{q}(\phi(\Omega))=-\frac{4}{\pi} \cos ^{2} \phi$ [25] so that the optimal power is obtained for (28),

$$
\left\{\begin{array}{c}
\Omega^{2}=1+k_{m}^{2}\left(1+\frac{2}{\pi} \sin 2 \phi\right) \\
k_{m}^{2} Q=\frac{\pi}{4} \frac{\Omega}{\cos ^{2} \phi}
\end{array}\right.
$$

which leads to (29). 


$$
\left\{\begin{array}{c}
\phi_{o p t}=-\arctan \left[\frac{Q\left(1+k_{m}^{2}-\Omega_{o p t}^{2}\right)}{\Omega_{o p t}}\right] \\
k_{m}^{2} Q=\frac{\pi}{4} \frac{\Omega_{o p t}}{\cos ^{2} \phi_{o p t}}
\end{array}\right.
$$

One can find the exact analytical expression of the optimal phase shift $\phi$ and the corresponding frequencies to harvest the maximum power. However, since the expressions are extremely large and hardly usable, we prefer to focus on the approximation :

$$
\left\{\begin{array}{c}
\phi_{o p t} \simeq-\arctan \left[\frac{Q\left(1+k_{m}^{2}-\Omega_{o p t}^{2}\right)}{\sqrt{1+k_{m}^{2}}}\right] \\
k_{m}^{2} Q \simeq \frac{\pi}{4} \frac{1}{\cos ^{2} \phi_{o p t}}
\end{array}\right.
$$

which gives (31), assuming $Q \gg 1$.

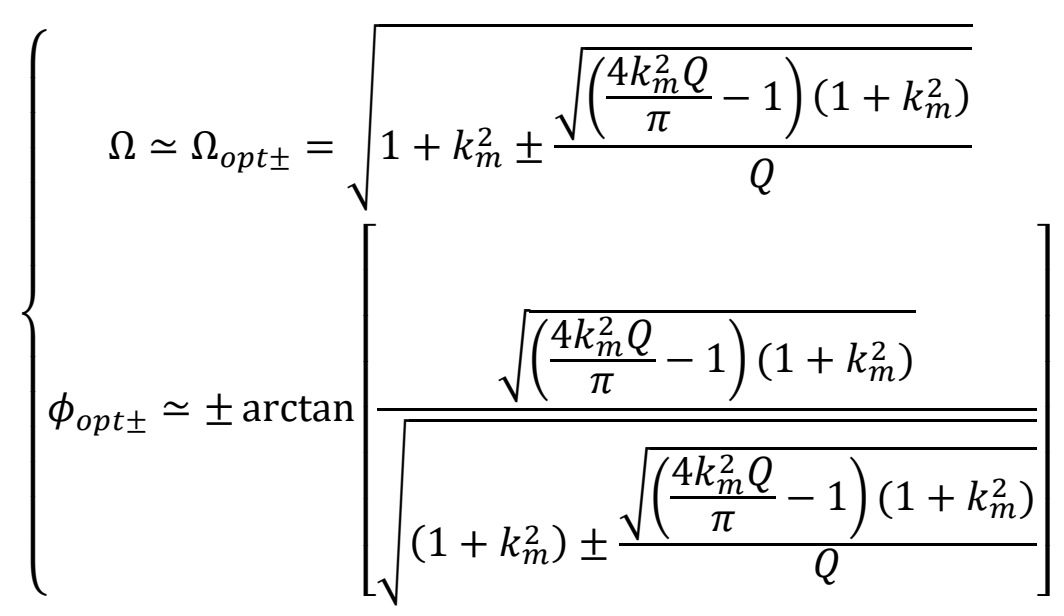

The solutions (31) only exist when (32) is fulfilled.

$$
k_{m}^{2} Q>\frac{\pi}{4}
$$

We find condition (32) which had been proposed but not demonstrated in a previous publication of PS SECE [25]. In addition, we get the expression of the optimal phase-shift to apply at the control stage.

\subsubsection{Tunable SECE technique}

In the case of tunable SECE, the parameter used for maximum power point tracking is the ratio between the peak piezoelectric voltage (when the electronic switch is closed) and the voltage after reopening the electronic switch. As noted in section 4.1, from the piezoelectric perspective, this situation is identical to SECPE. Hence, the corresponding waveforms are identical to Figure 9 and the optimal working conditions are (33), identical to (19). An integrated implementation of tunable SECE has been published recently [95]. It is called "synchronized-switch interface" but is actually based on a 2-stage topology (and not a 4-stage 
topology) with synchronized charge extraction and thus enters the category of SECE architectures.
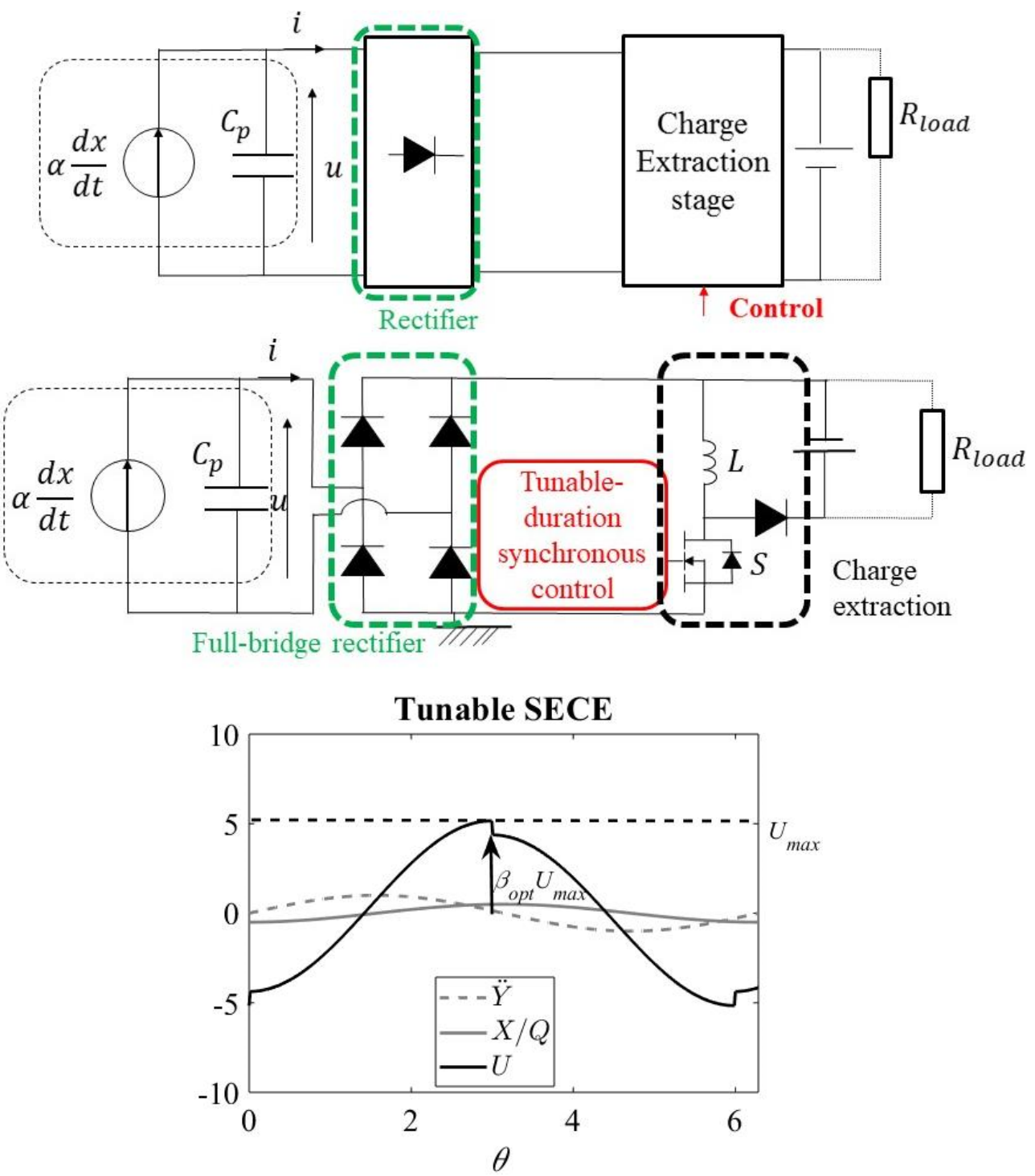

Figure 13: Topology (top), architecture (middle) and waveforms at the maximum power point (bottom) for the tunable SECE technique. Example for $\Omega=\Omega_{\text {opt }}, \beta=\beta_{\text {opt }}, k_{m}^{2}=0.1, Q=100$.

$$
\left\{\begin{array}{c}
\Omega=\Omega_{o p t}=\sqrt{1+k_{m}^{2}} \\
\beta=\beta_{o p t}=\frac{4 k_{m}^{2} Q-\pi \sqrt{1+k_{m}^{2}}}{4 k_{m}^{2} Q+\pi \sqrt{1+k_{m}^{2}}}
\end{array}\right.
$$

Hence, in the case of tunable SECE, the maximum power can be achieved at one single frequency $\Omega=\sqrt{1+k_{m}^{2}}$, under no constraint on $k_{m}^{2} Q$. These results are consistent with previous publications [24]. The architecture initially proposed for tunable SECE is given in Figure 13 but, to reach a negative value of $\beta$ (required to achieve the maximum power for weakly-coupled generators), the tunable SECE technique requires another architecture (for instance the one proposed for FTSECE in Figure 16). 


\subsubsection{Tunable USECE technique}

In the case of tunable USECE [51], the rectifier is made of a single shunt diode and the tuning parameter is the duration of charge extraction (see Figure 14). The corresponding voltage waveform at maximum power point is as depicted in Figure 14.
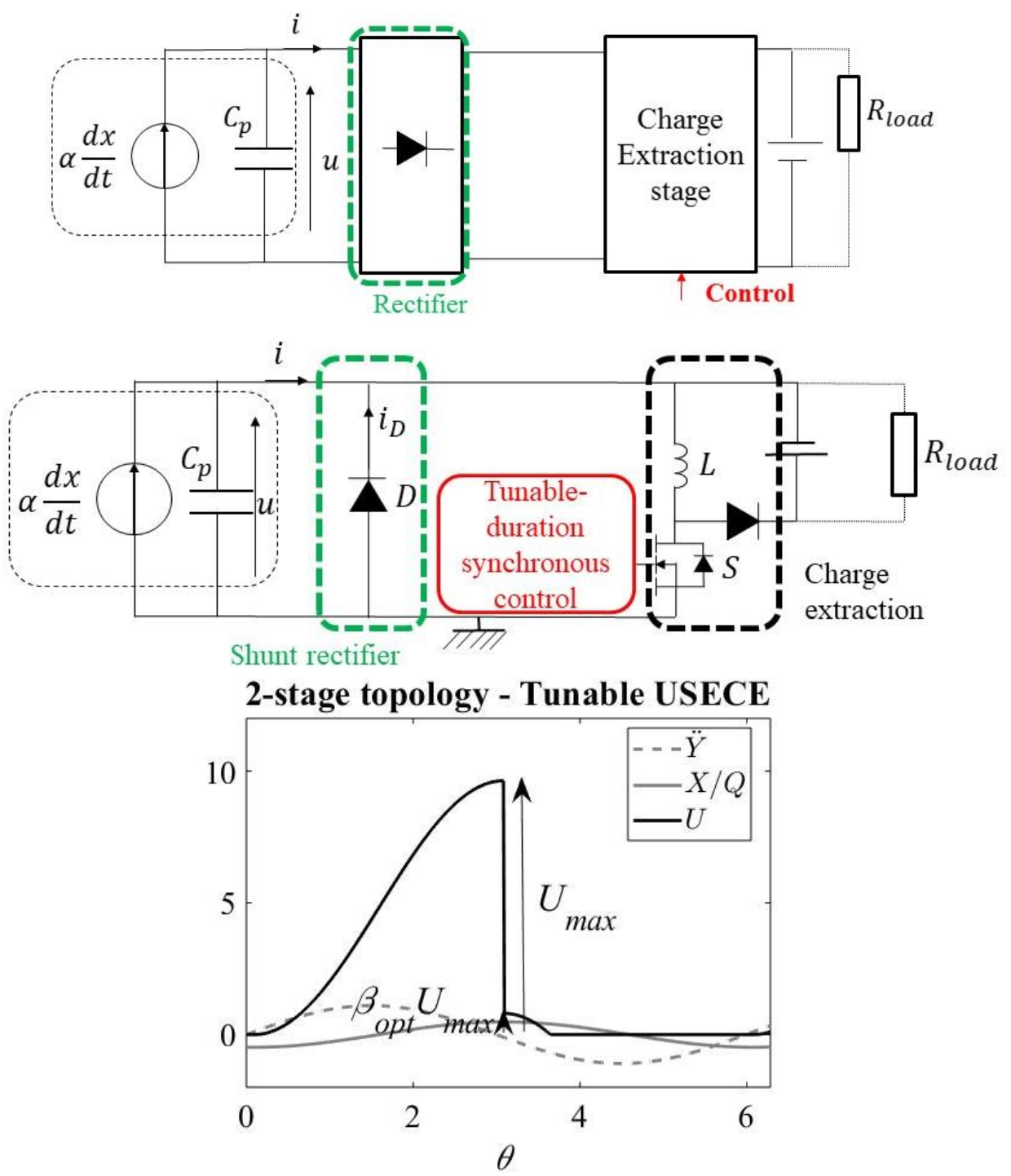

Figure 14: Topology (top), architecture (middle) and waveforms at the maximum power point (bottom) for the tunable unipolar SECE technique. Example for $\Omega=\Omega_{\text {opt }}, \beta=\beta_{\text {opt }}, k_{m}^{2}=0.1, Q=100$.

The in-phase and quadrature components of the piezoelectric voltage yield :

$$
\left\{\begin{array}{c}
U_{p}(\beta(\Omega))=\frac{2}{\pi}\left(1-\beta^{2}\right) \\
U_{q}(\beta(\Omega))=-\frac{1}{2}\left[1-\frac{2}{\pi}(1-2 \beta) \sqrt{\beta(1-\beta)}+\frac{1}{\pi} \arccos (1-2 \beta)\right]
\end{array}\right.
$$

So that the optimal frequency $\Omega_{\text {opt }}$ and tuning parameter $\beta_{\text {opt }}$ verify (35). 


$$
\left\{\begin{array}{c}
\Omega_{o p t}^{2}=1+k_{m}^{2} \frac{2}{\pi}\left(1-\beta_{o p t}^{2}\right) \\
k_{m}^{2} Q=\frac{\pi}{2} \frac{\Omega_{o p t}}{1-\frac{2}{\pi}\left(1-2 \beta_{o p t}\right) \sqrt{\beta_{o p t}\left(1-\beta_{o p t}\right)}+\frac{1}{\pi} \arccos \left(1-2 \beta_{o p t}\right)}
\end{array}\right.
$$

Unfortunately, this system cannot be solved analytically. However a second-order TaylorSeries expansion with respect to $\beta \simeq 1 / 2$ gives condition (36).

$$
k_{m}^{2} Q \geq \frac{3 \pi^{2}\left(27 \pi+2 \sqrt{3}(1+\pi)^{3 / 2}\right)}{\pi(\pi(231-4 \pi)-12)-4} \simeq \frac{\pi}{2}
$$

We find the condition found empirically in [51].

\subsubsection{N-SECE technique}

In the case of multiple N-SECE $(N>1)$, the tuning parameter is the number $N$ of charge extraction events which take place during each semi-period. Examples of waveforms at the maximum power point are depicted in Figure 15. If $N>1$, one can simplify the expressions given in [50] into (37).

$$
\forall N>1,\left\{\begin{array}{c}
U_{p}(N(\Omega))=1-\frac{N}{\pi} \sin \left(\frac{\pi}{N}\right) \\
U_{q}(N(\Omega))=\frac{N\left(\cos \left(\frac{\pi}{N}\right)-1\right)}{\pi}
\end{array}\right.
$$

One should be careful that the expression of $U_{q}$ is not valid in the specific case $N=1$ corresponding to classical SECE. As long as $N>1$, the condition to extract the maximum power is then (38).

$$
\left\{\begin{array}{c}
\Omega_{o p t}^{2}=1+k_{m}^{2}\left(1-\frac{N_{o p t}}{\pi} \sin \left(\frac{\pi}{N_{o p t}}\right)\right) \\
k_{m}^{2} Q=\frac{\Omega \pi}{N_{o p t}\left(1-\cos \left(\frac{\pi}{N_{o p t}}\right)\right)}
\end{array}\right.
$$

The minimum FOM for which the maximum power is achieved corresponds to classical SECE (see (39)).

$$
k_{m}^{2} Q \geq \frac{\pi}{4}
$$



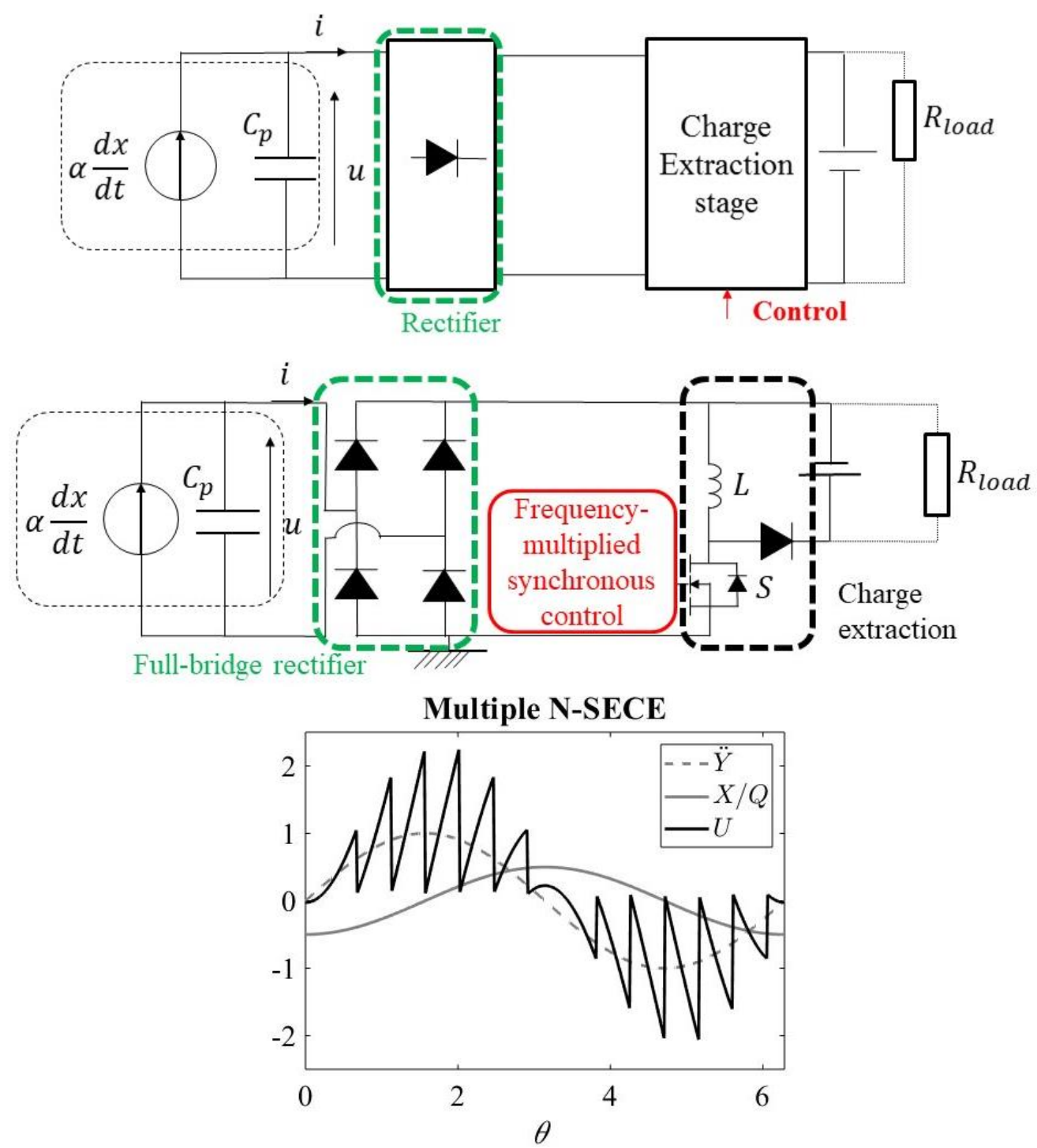

Figure 15: Topology (top), architecture (middle) and waveforms at the maximum power point (bottom) in the NSECE technique at the maximum power point. Example of multiple $N$-SECE for $\Omega=\Omega_{\text {opt }}, N \simeq N_{\text {opt }}=6, k_{m}^{2}=0.1$ and $Q=$ 100.

Without an additionnal assumption, system (38) cannot be treated for an arbitrary $N$ due to the complexity of the expressions obtained for $N>1$. However, a second-order Taylor-series expansion in $1 / N$ leads to (40)

$$
\left\{\begin{array}{c}
N=N_{o p t}=\frac{\pi k_{m} \sqrt{3 k_{m}^{2} Q^{2}-2}}{2 \sqrt{3}} \\
\Omega=\Omega_{o p t}=\frac{\sqrt{3} k_{m} Q}{\sqrt{3 k_{m}^{2} Q^{2}-2}}
\end{array}\right.
$$

In addition, for high-Q transducers, the solution is very simple (see (41)). 


$$
\left\{\begin{array}{c}
N=N_{o p t} \simeq \frac{\pi k_{m}^{2} Q}{2} \\
\Omega=\Omega_{o p t} \simeq 1
\end{array}\right.
$$

Hence, in multiple N-SECE, $P_{\text {lim }}$ is achieved for a frequency close to the natural frequency (short-circuit resonance frequency) and we obtain the corresponding optimal value of $N$.

In the regenerative case $(N<1)$ [50], charge extraction events take place every $1 / N$ period. The waveforms in this case are quite inconvenient to compare to the other strategies because one must plot $1 / N$ periods to observe the periodic pattern, $N$ being sometimes quite small (as demonstrated below). For this reason, we chose not to plot the piezoelectric waveform in this case. Nevertheless, other interesting conclusions and simple results can be deduced since $U_{p}=$ 1 and $U_{q}(N)=2 N\left((-1)^{1 / N}-1\right)$. Thus, we find that the maximum power point is obtained when (42) is fulfilled.

$$
\left\{\begin{array}{c}
\Omega_{o p t}^{2}=1+k_{m}^{2} \\
k_{m}^{2} Q=-\frac{\Omega_{o p t} \pi}{2 N_{o p t}\left((-1)^{1 / N_{o p t}}-1\right)}
\end{array}\right.
$$

If $1 / N$ is even, the condition (42) is never fulfilled and the maximum power is never achieved. However, if $1 / N$ is odd, the maximum power point is achieved for :

$$
N_{o p t}=\frac{\sqrt{1+k_{m}^{2}} \pi}{4 k_{m}^{2} Q}
$$

For high-Q transducers, we find that the condition for regenerative N-SECE to reach the maximum available power is (44).

$$
k_{m}^{2} Q>\pi / 4
$$

In this scenario, the maximum power is achieved at $\Omega_{o p t}=\sqrt{1+k_{m}^{2}}$. In the regenerative case, the larger $Q$ and/or $k_{m}^{2}$ are, the lower $N$ must be to harvest the maximum power. All these conclusions are consistent with previous results [50].

\subsubsection{FTSECE technique}

Interestingly, the topology of the FTSECE architecture is reversed compared to other SECEbased techniques. In FTSECE, the charge extraction stage comes before the rectifier. However, the resulting topology is still 2 stages and not 3 nor 4 stages, with only one synchronous control stage (see Figure 16), which explains why it is still called SECE and not hybrid SECE or SSH. The corresponding waveforms are depicted in Figure 16. 


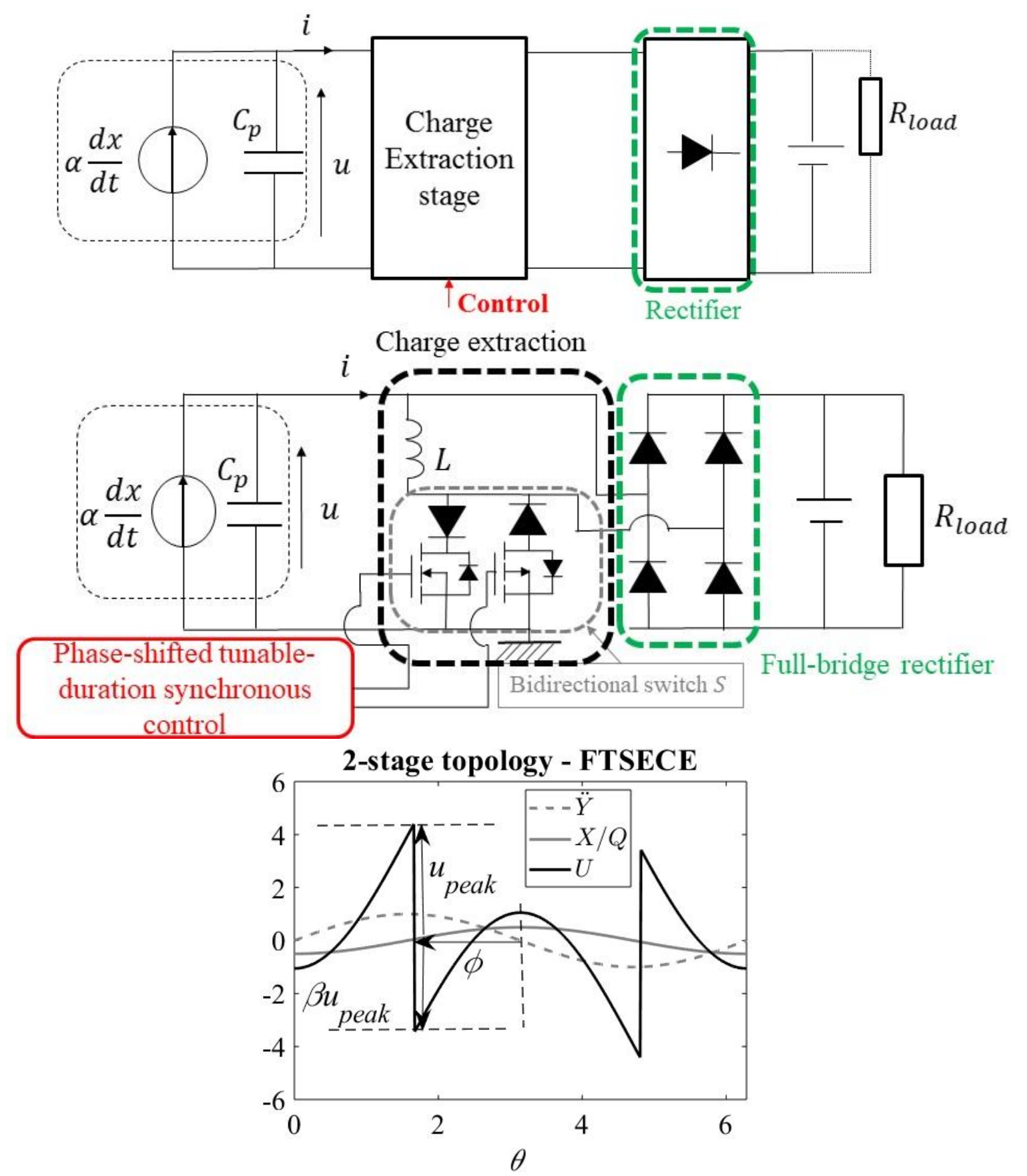

Figure 16: Topology (top), architecture (middle) and waveforms at the maximum power point (bottom) in the FTSECE technique at the maximum power point. Example for $\Omega=1, \beta=\beta_{\text {opt }}(<0), \phi=\phi_{\text {opt }}(<0), k_{m}^{2}=0.1$ and $Q=100$.

The two parameters used for power optimization are (i) the delay between the instant where the piezoelectric voltage reaches its peak and the instant of charge extraction, characterized by a phase-shift $\phi$ and (ii) the ratio between the piezoelectric voltage before and after charge extraction $\beta$ (see [47]). In such case:

$$
\left\{\begin{array}{c}
U_{p}(\beta(\Omega), \phi(\Omega))=1+\frac{2}{\pi} \frac{1-\beta}{1+\beta} \sin 2 \phi \\
U_{q}(\beta(\Omega), \phi(\Omega))=-\frac{4}{\pi} \frac{1-\beta}{1+\beta} \cos ^{2} \phi
\end{array}\right.
$$

The condition to reach the maximum power is then (46) 


$$
\left\{\begin{array}{c}
\Omega^{2}=1+k_{m}^{2}\left(1+\frac{2}{\pi} \frac{1-\beta_{o p t}}{1+\beta_{o p t}} \sin 2 \phi_{o p t}\right) \\
k_{m}^{2} Q=\frac{\Omega \pi\left(1+\beta_{o p t}\right)}{4\left(1-\beta_{o p t}\right) \cos ^{2} \phi_{o p t}}
\end{array}\right.
$$

which leads to (47).

$$
\left\{\begin{array}{c}
\beta(\Omega)=\beta_{\text {opt }}(\Omega)=\frac{4 k_{m}^{2} Q \Omega-\pi\left[\Omega^{2}+Q^{2}\left(1+k_{m}^{2}-\Omega^{2}\right)^{2}\right]}{4 k_{m}^{2} Q \Omega+\pi\left[\Omega^{2}+Q^{2}\left(1+k_{m}^{2}-\Omega^{2}\right)^{2}\right]} \\
\phi(\Omega)=\phi_{o p t}(\Omega)=-\arctan \left[\frac{Q\left(1+k_{m}^{2}-\Omega^{2}\right)}{\Omega}\right]
\end{array}\right.
$$

One can see that, in theory, the maximum power point is achievable for any frequency with such a technique. Some limitations to that ultimate performance have been discussed in [47]. They are mainly due to the inversion (magnetic and/or conduction) losses in the inductor which bound the values achievable by $\beta$.

\subsection{3-stage topologies}

4.3.1. FB 3-stage load adaptation technique (standard energy harvesting interface (SEH))

In the FB 3-stage load adaptation technique, the architecture and the displacement and voltage waveforms are as depicted in Figure 17.

The exact analytical expressions of the in-phase and quadrature components of the piezoelectric voltage can be found by combining the expression of the piezoelectric voltage given equation (11) of [65] with the relationship between $u_{c}$ and the first-harmonic amplitude $X_{1}$ given by equation (7) of [88]. The result is (48) (which has never been provided before).

$$
\left\{U_{p}(\Omega, r(\Omega))=\frac{\arccos \left[\frac{2 \pi}{\pi+2 r \Omega}-1\right]+\frac{\sqrt{2 \pi} \sqrt{r \Omega}}{\left(r \Omega+\frac{\pi}{2}\right)^{2}}\left(r \Omega-\frac{\pi}{2}\right)}{U_{q}(\Omega, r(\Omega))=-\frac{2 r \Omega}{\left(r \Omega+\frac{\pi}{2}\right)^{2}}}\right.
$$

An approached expression of the in-phase and quadrature component of the piezoelectric voltage with respect to the motion of the transducer can be found in [26] or [88]. This expression is based on the assumption that, if $x(t)$ is written in the form $x(t)=x_{m} \sin (\Omega t-\theta)$ then the piezoelectric voltage can be written $U(t)=g(\omega t-\theta)$ where $g$ is a $2 \pi$-periodic function. The results of this approximation are reasonable. This approximation is mathematically equivalent to (49) and the corresponding equations can be found in [88].

$$
\arccos \left[\frac{2 \pi}{\pi+2 r \Omega}-1\right] \simeq f(r \Omega)=\frac{2 \pi(\pi-2 r \Omega)}{(\pi+2 r \Omega)^{2}} \sqrt{\frac{2 r \Omega}{\pi}}-\pi\left(\frac{2 r \Omega}{\pi+2 r \Omega}\right)
$$




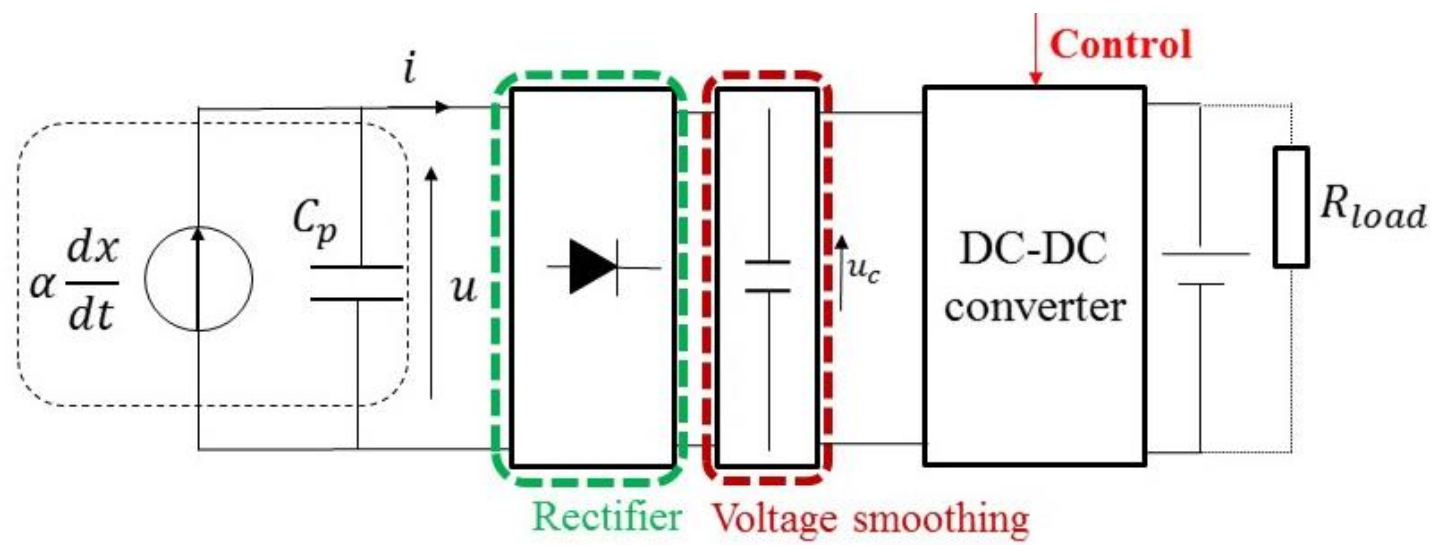

Smoothing capacitor $C_{\text {rect }}$

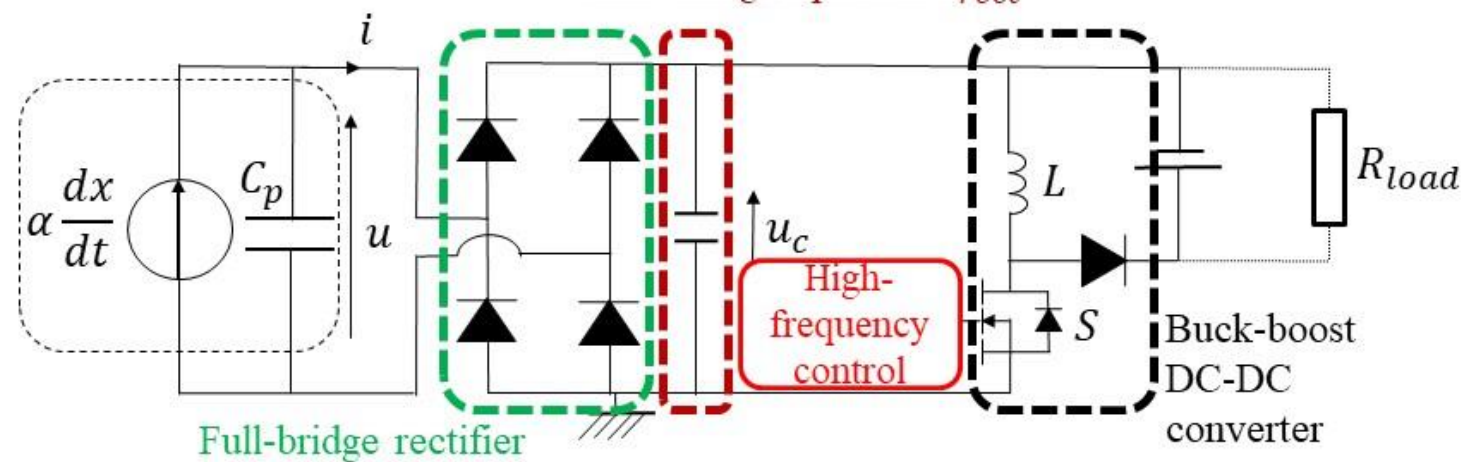

FB 3-stage load adaptation
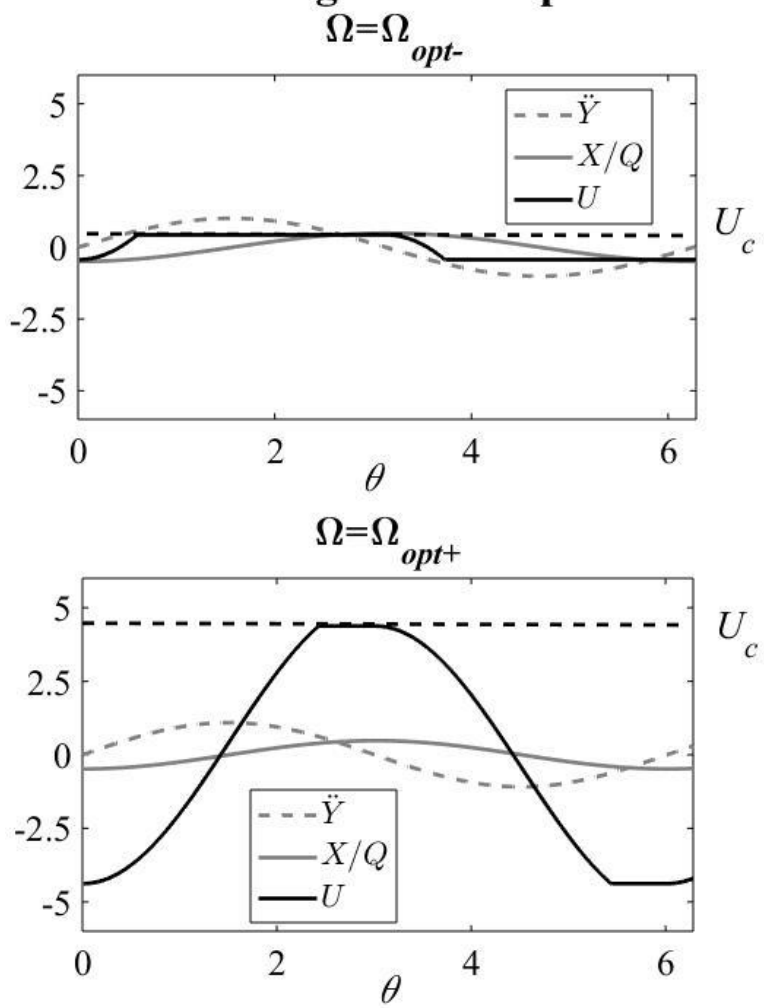

Figure 17: Topology (top), architecture (middle) and waveforms at the maximum power point (bottom) in the FB 3-stage load adaptation technique. Example for $\Omega=\Omega_{\text {opt+ }}, r=r_{\text {opt }}, k_{m}^{2}=0.1, Q=100$. 
We show, in Figure 18, that the error brought by (49) is bounded on a large range of $r \Omega$ (although it reaches $-23 \%$ for very low values of $\Omega(\simeq 0.1)$. In most applications, the optimal value of $r \Omega$ is between 1 and 10 and the approximation becomes good (less than $8.3 \%$ relative error).
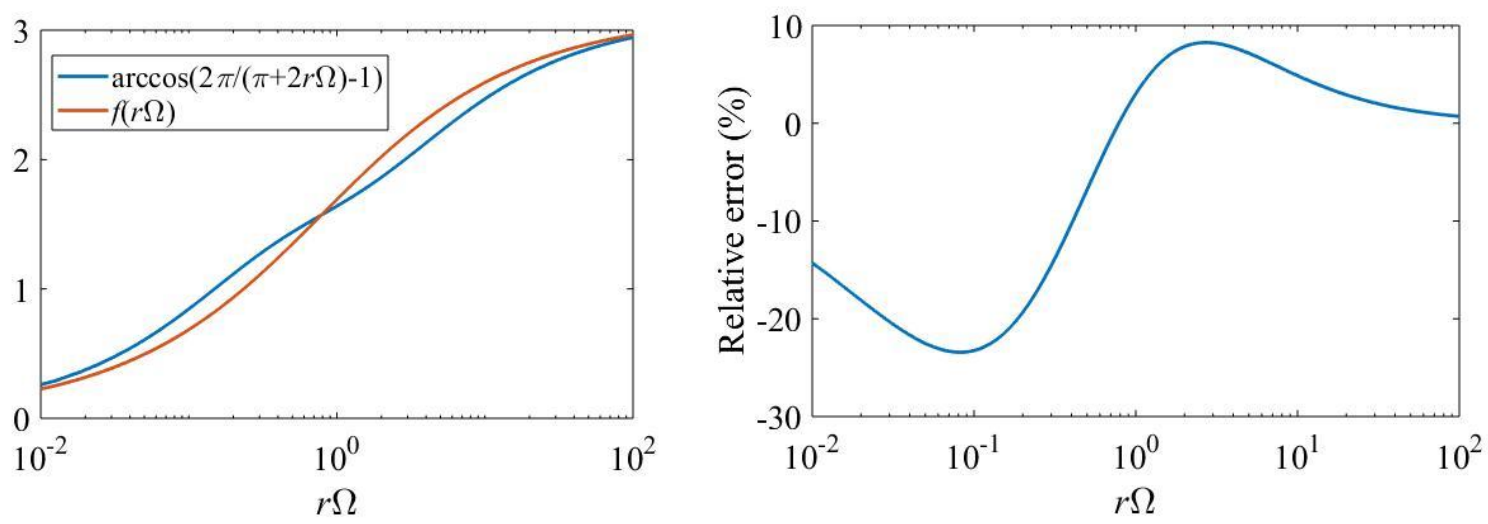

Figure 18: Error generated by the approximation proposed by Shu and Lien [88].Left : Approximation. Right : Relative error.

Given (48) and (49), the condition to reach the maximum power is (50).

$$
\left\{\begin{array}{c}
\Omega_{o p t}^{2}=1+k_{m}^{2}\left(\frac{\Omega_{o p t} r_{o p t}}{r_{o p t} \Omega_{o p t}+\frac{\pi}{2}}\right) \\
k_{m}^{2} Q=\frac{\left(r_{o p t} \Omega_{o p t}+\frac{\pi}{2}\right)^{2}}{2 r}
\end{array}\right.
$$

The general solution of (50) with respect to $\Omega$ and $r$ can be found almost instantly with a symbolic calculation software such as Wolfram Mathematica but it is so large and complex that it has little interest. However, for $Q \gg 1, \Omega_{o p t} \simeq \sqrt{1+\frac{k_{m}^{2}}{2}}$ and one can approximate the system by $(51)$.

$$
\left\{\begin{array}{c}
\Omega_{o p t}^{2}=1+k_{m}^{2}\left(\frac{\Omega_{o p t} r_{o p t}}{r_{o p t} \Omega_{o p t}+\frac{\pi}{2}}\right) \\
k_{m}^{2} Q \simeq \frac{\sqrt{1+\frac{k_{m}^{2}}{2}}\left(r_{o p t} \Omega_{o p t}+\frac{\pi}{2}\right)^{2}}{2 r_{o p t} \Omega_{o p t}}
\end{array}\right.
$$

The condition to reach the maximum power is thus (52).

$$
k_{m}^{2} Q \geq \frac{\pi^{2}}{4 Q}+\pi \sqrt{1+\frac{\pi^{2}}{16 Q^{2}}}
$$

In this case, the optimal frequency and load verify (53). 


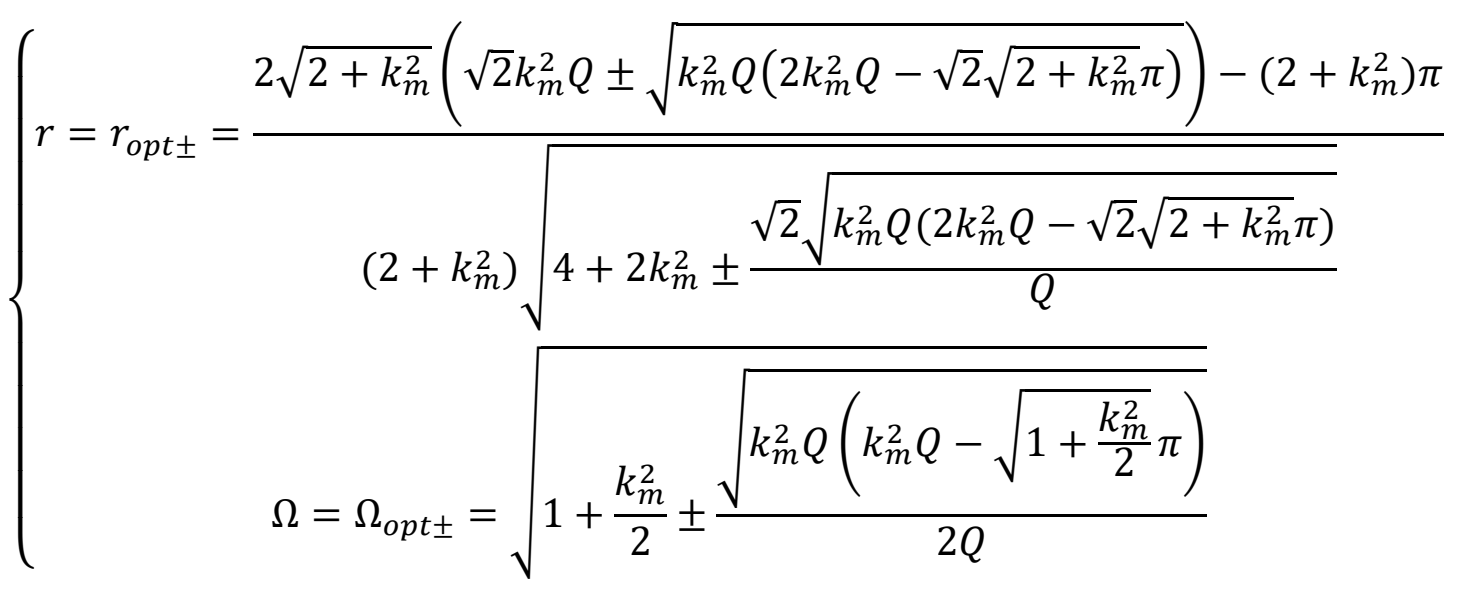

In the case where $Q \gg 1$, we find the condition $k_{m}^{2} Q \geq \pi$. The empirical condition $k_{m}^{2} Q \geq 5$ given by Shu and Lien [88] would correspond to a relatively low-Q scenario (worst-case scenario) with $Q \simeq 1.6$.

Several implementations of MPPT for this architecture [96] [97] [98] and a comparison between their performances can be found in recent literature dedicated to circuit optimization [98].

\subsubsection{HB 3-stage load adaptation technique}

In the case of 3-stage load adaptation with a HB rectifier instead of a FB rectifier, the waveforms are as depicted in Figure 19. They are identical to the FB 3-stage load adaptation technique except that the piezoelectric voltage is always positive, due to the voltage doubling effect. 


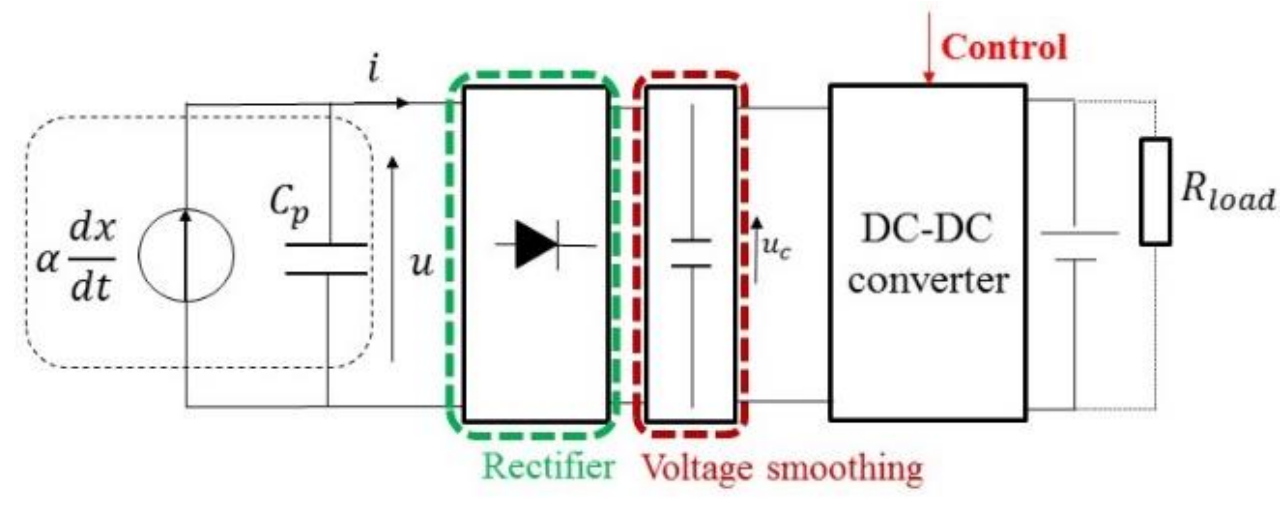

Smoothing capacitor $C_{\text {rect }}$

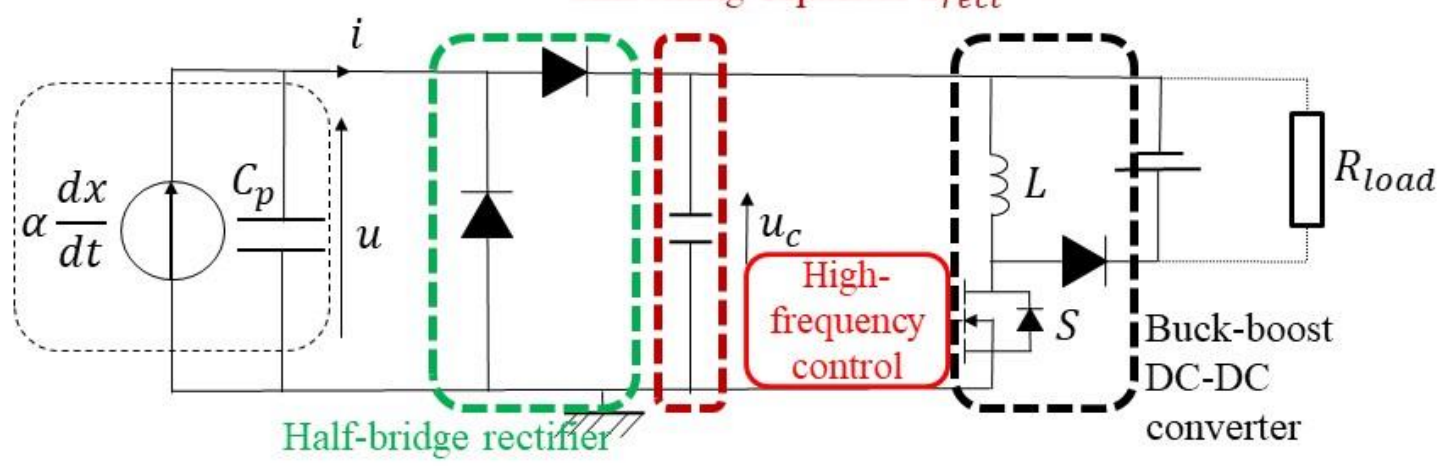

HB 3-stage load adaptation
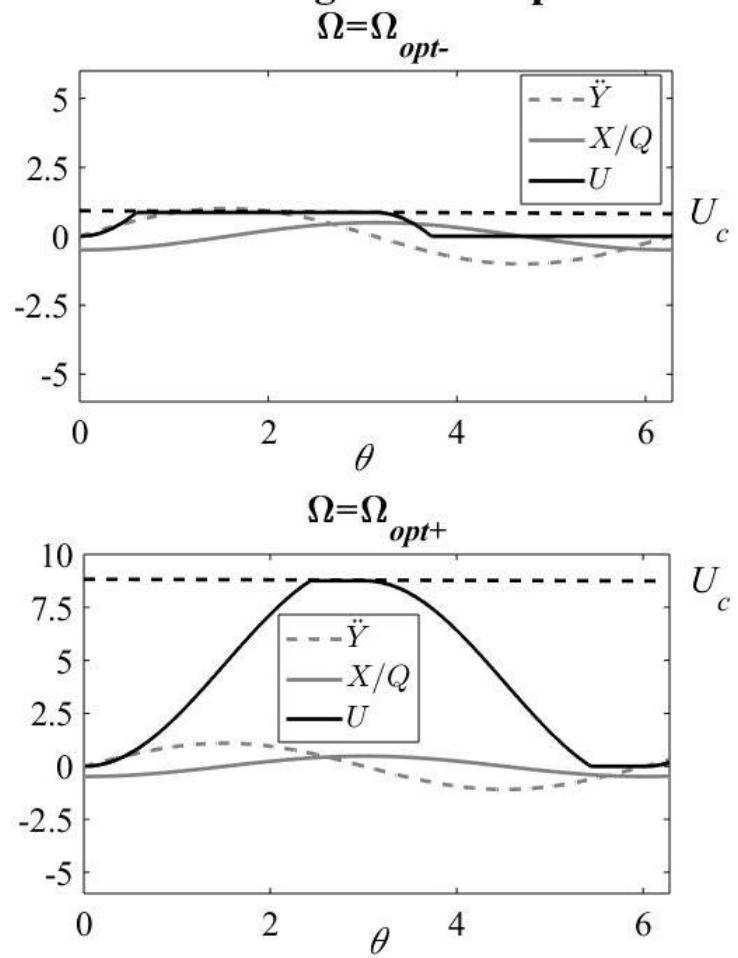

Figure 19: Topology (top), architecture (middle) and waveforms at the maximum power point (bottom) in the HB 3-stage load adaptation technique. Example for $\Omega=\Omega_{\text {opt }}, r=r_{\text {opt }} k_{m}^{2}=0.1, Q=100$.

Based on the same power balance as in 4.3.1, a power balance analysis [88] yields $U_{c}=\frac{2 r \Omega X_{m}}{2 \pi+r \Omega}$. The harmonic balance based on the waveforms of Figure 19 yield the expressions of the inphase and quadrature components of the piezoelectric voltage which verify (54). 


$$
\left\{\begin{array}{c}
U_{p}(\Omega, r(\Omega))=\frac{\arccos \left[\frac{2 \pi}{\pi+\frac{r}{2} \Omega}-1\right]+\frac{\sqrt{2 \pi} \sqrt{\frac{r}{4} \Omega}\left[\frac{r \Omega}{4}-\frac{\pi}{2}\right]}{\left(\frac{\pi}{2}+\frac{r \Omega}{4}\right)^{2}}}{U_{q}(\Omega, r(\Omega))=-\frac{r \Omega}{2\left(\frac{r \Omega}{4}+\frac{\pi}{2}\right)^{2}}}
\end{array}\right.
$$

A comparison between (54) and (48) shows that we obtain the same expressions as for the FB 2 -stage load adaptation case, apart from the resistance $r$ which is substituted by $r / 4$. Hence, the same power is obtained than in the FB case with an optimal resistance 4 times larger. The condition on $k_{m}^{2} Q$ to reach the maximum power is thus the same as (52). If $Q \gg 1$, we find again $k_{m}^{2} Q \geq \pi$.

4.3.3. Tunable SCSECE technique

Previous literature proposes one technique corresponding to 2-parameter optimization in a 3stage topology. This technique is the tunable SCSECE, where one can adjust (i) the instant of charge extraction (phase-shift $\phi_{S}$ ) and (ii) the duration of the phase where the piezoelectric transducer is short-circuited (characterized by an angular time $\Delta \phi$ ) [53].

In such case, we obtain:

$$
\left\{\begin{array}{l}
U_{p}\left(\phi_{s}(\Omega), \Delta \phi(\Omega)\right)=1-\frac{\Delta \phi}{\pi}+\frac{\sin \left(2\left(\phi_{s}+\Delta \phi\right)\right)}{2 \pi}+\frac{\sin \left(2 \phi_{s}\right)}{2 \pi}+\frac{2 \cos \left(\phi_{s}+\Delta \phi\right) \sin \left(\phi_{s}\right)}{\pi} \\
U_{q}\left(\phi_{s}(\Omega), \Delta \phi(\Omega)\right)=-\frac{1}{\pi}\left[\cos \left(\phi_{s}\right)+\cos \left(\phi_{s}+\Delta \phi\right)\right]^{2}=-\frac{2}{\pi} \cos ^{2}\left(2 \phi_{s}+\Delta \phi\right) \cos ^{2}(\Delta \phi)
\end{array}\right.
$$

The condition to reach the maximum power becomes:

$$
\left\{\begin{array}{c}
\Omega_{o p t}^{2}=1+k_{m}^{2}\left(1-\frac{\Delta \phi_{o p t}}{\pi}+\frac{\sin \left(2\left(\phi_{s_{o p t}}+\Delta \phi_{o p t}\right)\right)}{2 \pi}+\frac{\sin \left(2 \phi_{s_{o p t}}\right)}{2 \pi}+\frac{2 \cos \left(\phi_{s_{o p t}}+\Delta \phi_{o p t}\right) \sin \left(\phi_{s_{o p t}}\right)}{\pi}\right) \\
k_{m}^{2} Q=\frac{\Omega_{o p t} \pi}{\left(\cos \left(\phi_{s_{o p t}}+\Delta \phi_{o p t}\right)+\cos \left(\phi_{s_{o p t}}\right)\right)^{2}}
\end{array}\right.
$$

The usual approximation $\Omega \simeq 1$ in the second equation yields :

$$
\Delta \phi_{o p t}=\arccos \left[\sqrt{\frac{\pi}{k_{m}^{2} Q}}-\cos \phi_{s_{o p t}}\right]-\phi_{s_{o p t}}
$$

One can verify that (57) is consistent with previously-published results [53]. Unfortunately, there is no generic analytical expression of the optimal solution of (56) and only a numerical resolution is mathematically feasible. For weak coupling cases, $\phi_{s_{\text {opt }}}=0$ and $\Delta \phi_{s_{\text {opt }}}=0$, so that the tunable SC-SECE technique is equivalent to classical SECE. The maximum power can then only be achieved, for $Q \gg 1$, when $k_{m}^{2} Q \geq \frac{\pi}{4}$. 

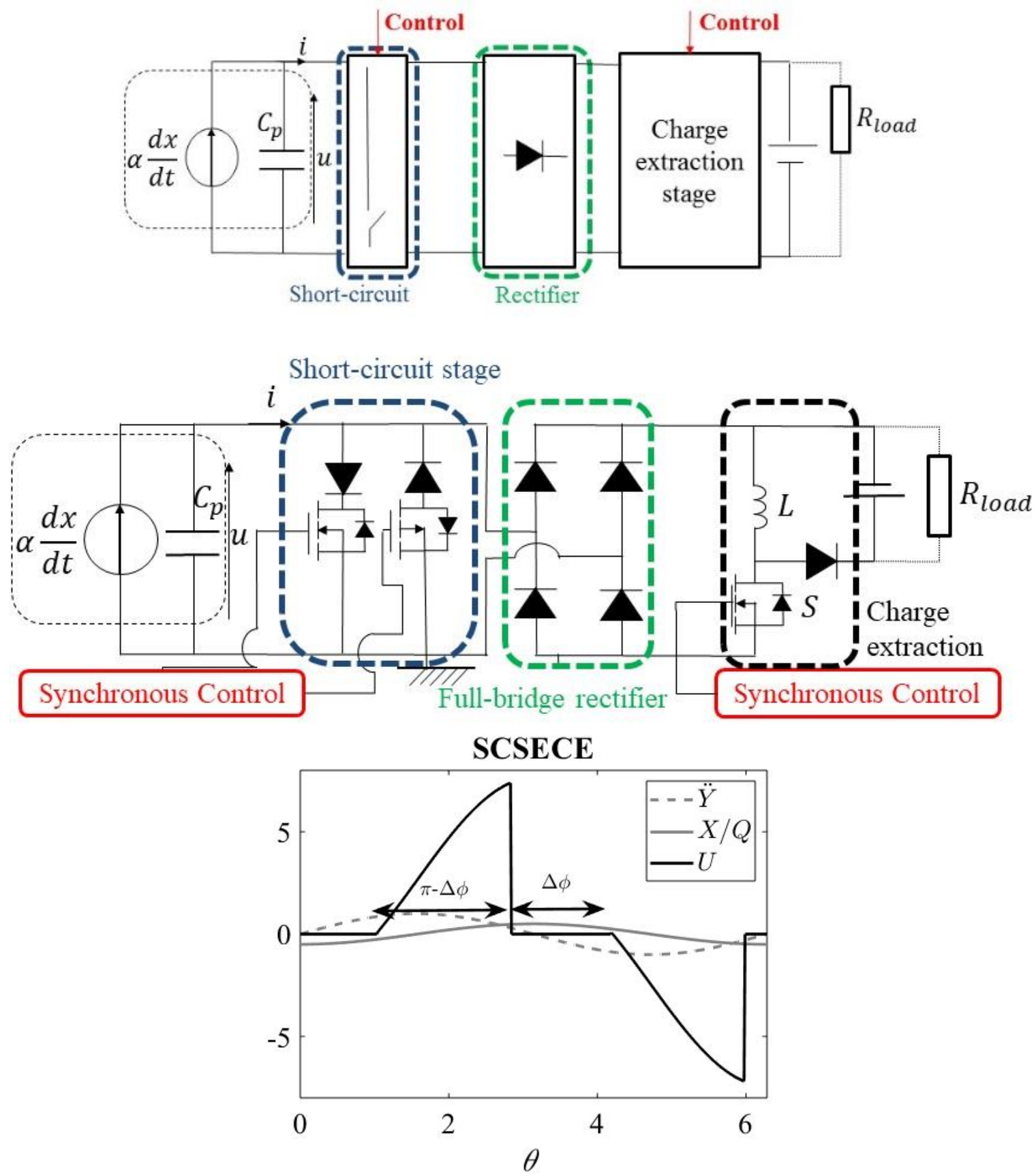

Figure 20: Topology (top), architecture (middle) and waveforms at the maximum power point (bottom) in the SCSECE technique. Example for $=1, \phi_{s}=\phi_{s_{o p t}}, \Delta \phi=\Delta \phi_{\text {opt }}, k_{m}^{2}=0.1, Q=100$.

\subsection{4-stage topology : the SSH architecture}

The only 4-stage topology that one can find, up to now, in the scientific literature is the SSH architecture. It has several variants like parallel SSHI, series SSHI, ESSH and DSSH, SSHC or, very recently, SSH-t "Synchronized Switch Harvesting on Inductor with threshold detection" [64]. In terms of piezoelectric voltage, DSSH and ESSH are identical to series SSHI. They only consist in the different implementation of the optimal load emulation so that one can treat them similarly. Autonomous (sometimes integrated circuit) implementations of SSH techniques (like "SP-SSHI" for "self-powered synchronized switch harvesting on inductor", "ISP-SSHI" for its "improved" version [61] [58] or COV-PSSHI for "controllable voltage" [56]) have already been published [61] [99] [100] [101] [102]. Often, the SSHC technique replaces the inductor by switched capacitors, for integrated circuit implementation [100] [101]. 
Other successful attempts replace the inductor by a piezoelectric oscillator [62] (the result being called SSHO for "Synchronized Switch Harvesting on Oscillator") or the electronic switches by mechanical switches (resulting in SSHI-SAMS for "Self-Adaptive Mechanical Switches") [63]. The maturity of SSH is already quite good and latest works focus on optimized circuits more than proofs of concepts or theoretical investigations. For example, a recent study focused on minimizing the number of components for control with a peak detector based on a capacitive divider [103]. Recent papers have even adapted SSHI for energy harvesting from shocks [104]. Another one (about SSH-t for "Synchronized Switch Harvesting on Inductor with threshold detection") has proposed a solution for weakly-coupled generators to guarantee that switching events occur only when the piezoelectric voltage is sufficiently high [64].

Independently of all these implementations that address specific practical issues, we sum up, in this section, the topology, waveform and principle of SSH-based systems and draw conclusions based on our analysis.

\subsubsection{Parallel SSHI (p-SSHI) technique}

In the case of parallel SSHI and its derivatives, the waveforms are depicted in Figure 21, along with the corresponding topology and architecture. In some implementations, the voltage inversion is operated by a fast succession of small steps to minimize energy losses and optimize the inversion [105]. One of these implementations of P-SSHI with successive switching steps was called P-S3BF for "parallel synchronized triple bias-flip" interface circuit [59]. Its version with a tunable delay for the switching events was called PV-P-S3BF for "Phase-variable parallel synchronized triple bias-flip" [60] [45]. Another version with tunable switch duration was called $\mathrm{S}^{3} \mathrm{HI}$ (for "Surge-inducing Synchronized-Switch Harvesting on Inductor") and its version with multiple-step voltage inversion $\mathrm{H}-\mathrm{S}^{3} \mathrm{HI}$ (for "High-frequency-included SurgeInducing Synchronized-Switch Harvesting on Inductor") [57].

Independently of the implementation, the expression (58) of $U_{p}$ and $U_{q}$ can be found from [72], where $q_{1}$ is the inversion factor (see Figure 21), dependent on the quality factor of the inductor and on other energy losses (an ideal inversion would correspond to $q_{1}=1$ ).

$$
\left\{\begin{array}{c}
U_{p}(r(\Omega), \Omega)=\frac{\frac{1-q_{1}}{2} r \Omega}{\frac{1-q_{1}}{2} r \Omega+\frac{\pi}{2}}=\frac{\left(1-q_{1}\right) r \Omega}{\left(1-q_{1}\right) r \Omega+\pi} \\
U_{q}(r(\Omega), \Omega)=-\frac{2\left[1+\frac{r \Omega}{2 \pi}\left(1-q_{1}^{2}\right)\right] r}{\left(\frac{1-q_{1}}{2} r \Omega+\frac{\pi}{2}\right)^{2}}
\end{array}\right.
$$

Thus, the condition to reach the maximum power is:

$$
\left\{\begin{array}{l}
\Omega_{o p t}^{2}=1+k_{m}^{2}\left(\frac{\left(1-q_{1}\right) r_{o p t} \Omega_{o p t}}{\left(1-q_{1}\right) r_{o p t} \Omega_{o p t}+\pi}\right) \\
k_{m}^{2} Q=\frac{\pi \Omega_{o p t}\left(\left(1-q_{1}\right) r_{o p t} \Omega_{o p t}+\pi\right)^{2}}{4\left[2 \pi+r_{o p t} \Omega_{o p t}\left(1-q_{1}^{2}\right)\right] r_{o p t}}
\end{array}\right.
$$




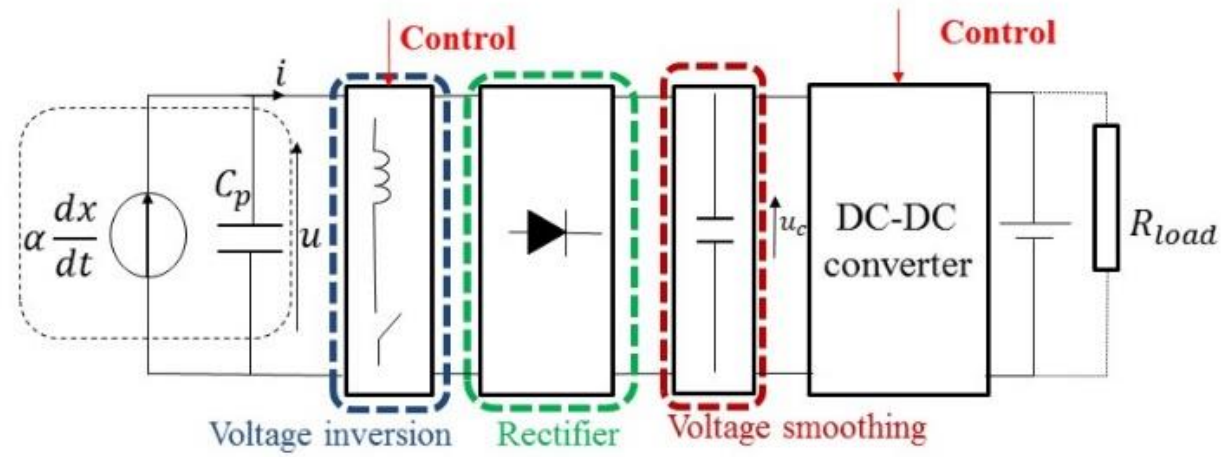

Voltage inversion Full-bridge rectifier Smoothing capacitor $C_{\text {rect }}$

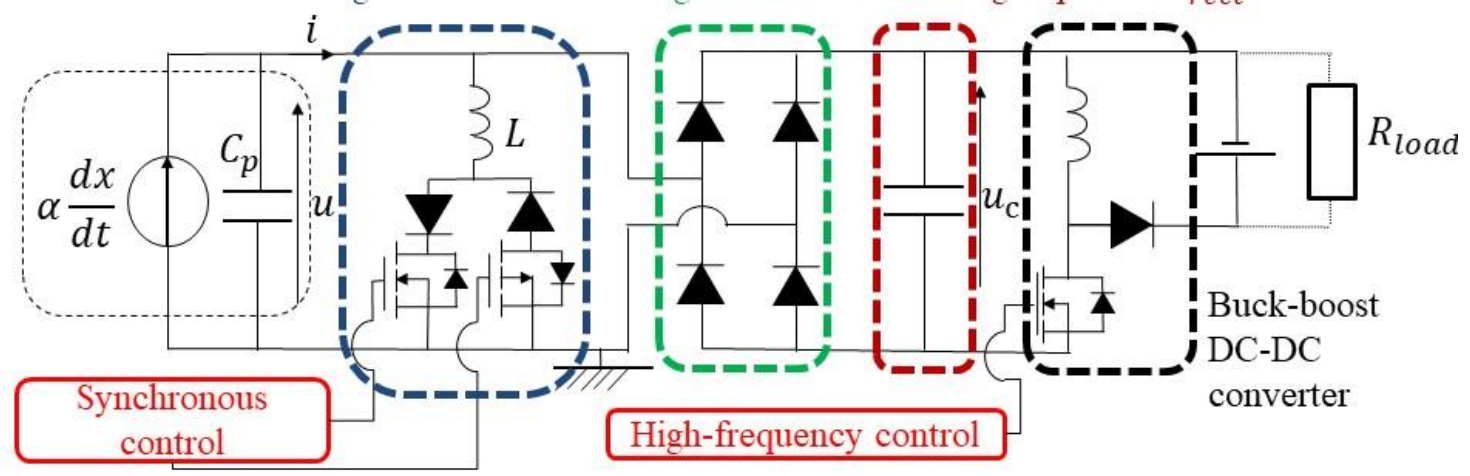

Parallel SSHI

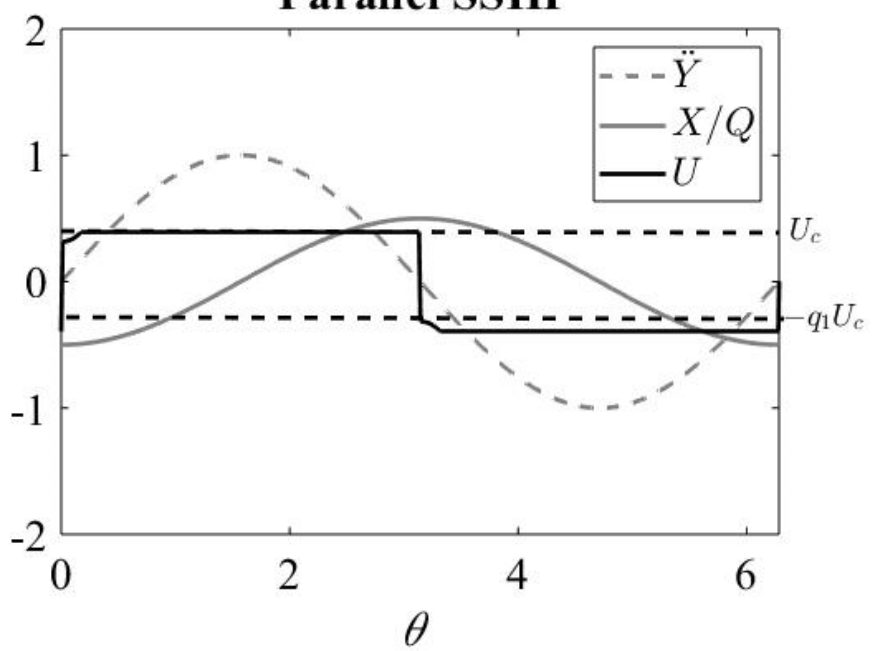

Figure 21: Topology (top), architecture (middle) and waveforms (bottom) at the maximum power point (bottom) in the parallel-SSHI technique. Example for $q_{1}=0.8$ (corresponding optimal frequency: $\Omega=\Omega_{\text {opt }}, k_{m}^{2}=0.1, Q=100$.

Solving this system is feasible but the expression is extremely large. For $Q \gg 1$, the solution is (60).

$$
\left\{\begin{array}{c}
r=r_{o p t} \simeq \frac{\pi k_{m}^{2}\left[(\pi+4 Q) \sqrt{\left(8-\frac{\pi}{Q}\left(1-q_{1}\right)\right)^{2}-\frac{16 \pi}{k_{m}^{2} Q}\left(1-q_{1}\right)^{2}}+\left(\frac{8 \pi}{k_{m}^{2}}-\frac{\pi^{2}}{Q}\right)\left(1-q_{1}\right)-4\left(8 Q-\pi\left(1+q_{1}\right)\right)\right]}{8\left(1+2 k_{m}^{2}-q_{1}\right)\left(4 k_{m}^{2} Q\left(1+q_{1}\right)-\left(1+k_{m}^{2}\right) \pi\left(1-q_{1}\right)\right)} \\
\Omega=\Omega_{o p t} \simeq \sqrt{1+\frac{k_{m}^{2}}{1-q_{1}}\left[1-\sqrt{1-\frac{\pi}{4 k_{m}^{2} Q}\left(1-q_{1}\right)^{2}}\right]}
\end{array}\right.
$$


Parallel-SSHI can thus reach the maximum power as long as the coupling is sufficient for (61) to be satisfied. Previous studies have provided the expression of the optimal duty-cycle (and thus $r_{o p t}$ ) as a function of the electrical variables (voltages and currents) [106]

$$
k_{m}^{2} Q \geq \frac{\pi\left(1-q_{1}\right)^{2}}{4}
$$

If the inversion is ideal $\left(q_{1}=1\right)$, one finds the condition (62) expressed in previous studies [72].

$$
\left\{\begin{array}{c}
r=r_{o p t}=\frac{\pi^{2}}{8 k_{m}^{2} Q} \\
\Omega=\Omega_{o p t}=1
\end{array}\right.
$$

In such case, there is no condition on the coupling coefficient. However, the lower the quality factor of the inductor used for the inversion is, the higher the coupling should be to extract the maximum power. One can also check that, the lower $q_{1}$ is, the higher $\Omega_{o p t}$ is. All these results are also consistent with the only multi-parameter technique for a 4-stage topology, which has been called the tunable (hybrid p-)SSHI technique [107].

\subsubsection{Series SSHI (s-SSHI), DSSH and ESSH techniques}

In the series SSHI, DSSH and ESSH techniques, the voltage is inverted prior to the rectifier stage (either by an inductor stage, or by a switched capacitor cell emulating the behavior of an inductor (in SSHC)). From the piezoelectric perspective all these techniques behave equivalently, the main difference between SSHI, DSSH and ESSH being in the technological solution to emulate the output load $r_{e q}$.

The normalized piezoelectric voltage after inversion is written $U_{m}$ (see Figure 22). Writing $U_{c}$ the normalized voltage across the smoothing capacitor placed at the output of the full-bridge rectifier (see Figure 6), the inversion factor $q_{1}$ verifies (63).

$$
q_{1}=\frac{U_{m}-U_{c}}{U_{\max }+U_{c}}
$$

Based on [72], the in-phase and quadrature components of the piezoelectric voltage are written (64).

$$
\left\{\begin{array}{c}
U_{p}(r(\Omega), \Omega)=1 \\
U_{q}(r(\Omega), \Omega)=-\frac{4\left(1+q_{1}\right)}{\left(1-q_{1}\right) \pi+2 r \Omega\left(1+q_{1}\right)}
\end{array}\right.
$$

Hence, the condition to reach the maximum power is (65).

$$
\left\{\begin{array}{c}
\Omega_{o p t}^{2}=1+k_{m}^{2} \\
k_{m}^{2} Q=\frac{\Omega_{o p t}\left(\left(1-q_{1}\right) \pi+2 r_{o p t} \Omega_{o p t}\left(1+q_{1}\right)\right)}{4\left(1+q_{1}\right)}
\end{array}\right.
$$




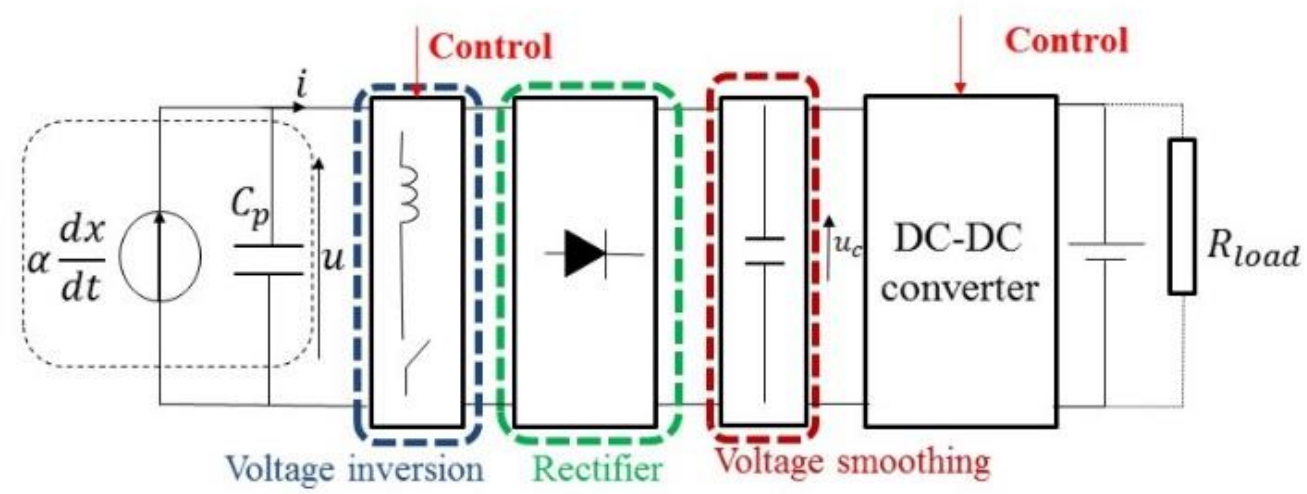

Voltage inversion Full-bridge rectifier Smoothing capacitor $C_{\text {rect }}$
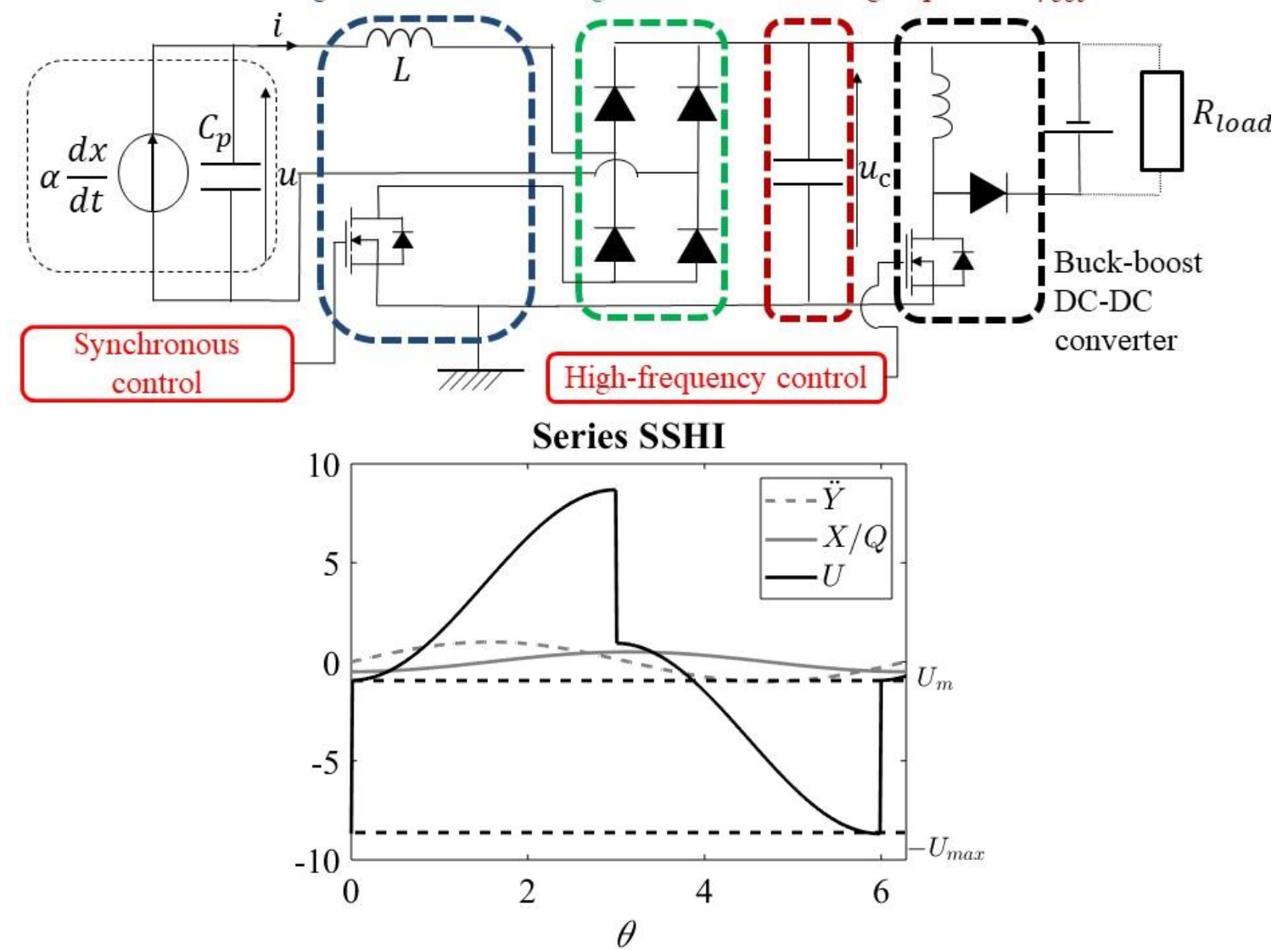

Figure 22: Normalized acceleration $\ddot{Y}$, piezoelectric voltage $U$ and displacement $X$ waveforms in the series SSHI technique. Example for $\Omega=\Omega_{\text {opt }}, q_{1}=0.8, k_{m}^{2}=0.1, Q=100$.

The maximum power can only be achieved for one single frequency $\Omega_{o p t}=\sqrt{1+k_{m}^{2}}$ and the corresponding value of the load resistance is then (66).

$$
r_{o p t}=\frac{4 k_{m}^{2}\left(1+q_{1}\right) Q-\pi\left(1-q_{1}\right) \sqrt{1+k_{m}^{2}}}{2\left(1+k_{m}^{2}\right)\left(1+q_{1}\right)}
$$

For a given inversion level $q_{1}$, a necessary condition to reach the maximum power is then :

$$
k_{m}^{2} Q>\frac{\pi\left(\pi\left(1-q_{1}\right)^{2}+\left(1-q_{1}\right) \sqrt{\pi^{2}\left(1-q_{1}\right)^{2}+64 Q^{2}\left(1+q_{1}\right)^{2}}\right)}{32 Q\left(1+q_{1}\right)^{2}}
$$

For $Q \gg 1$, this condition becomes (68). 


$$
k_{m}^{2} Q \geq \frac{\pi\left(1-q_{1}\right)}{4\left(1+q_{1}\right)}
$$

An interesting conclusion can be drawn from the comparison between (68) and (61) : parallel SSHI is able to extract more power for generators with weaker coupling than series SSHI.

In the ideal case of perfect inversion $q_{1}=1$, the maximum power can be achieved for any value of $k_{m}^{2} Q$ and the corresponding frequency and load are, as given in [72] :

$$
\left\{\begin{array}{c}
r_{o p t}=\frac{2 k_{m}^{2} Q}{1+k_{m}^{2}} \\
\Omega_{o p t}=\sqrt{1+k_{m}^{2}}
\end{array}\right.
$$

As done for parallel SSHI in a recent study [107], one could imagine tuning the inversion factor $q_{1}$ at a fixed output load $r$. Such strategy would give the optimal inversion factor $q_{o p t}$ at $\Omega_{o p t}=$ $\sqrt{1+k_{m}^{2}}$ given in (70).

$$
q_{\text {opt }}=\frac{\sqrt{1+k_{m}^{2}} \pi-4 k_{m}^{2} Q+2 r\left(1+k_{m}^{2}\right)}{\sqrt{1+k_{m}^{2}} \pi+4 k_{m}^{2} Q-2 r\left(1+k_{m}^{2}\right)}
$$

5. Summary

\subsection{Main results}

The main results of this review are summed up in Table III. 
Table III. Characteristics of existing techniques allowing adaptive control strategies for $Q \gg 1$

\begin{tabular}{|c|c|c|c|c|c|c|c|}
\hline $\begin{array}{l}\text { Nr. of } \\
\text { stages }\end{array}$ & Architecture & Technique & $\begin{array}{l}\text { Waveforms at maximum power } \\
\text { points }\end{array}$ & $\begin{array}{l}\text { Switch control } \\
\text { stages }\end{array}$ & $\Omega_{o p t}^{2}\left(\right.$ or $\Omega_{o p t \pm}^{2}$ if 2 solutions $)$ & Optimal parameters & $\begin{array}{l}k_{m}^{2} Q \\
\text { lower } \\
\text { bound }\end{array}$ \\
\hline 1 & OSECE & SECPE & \begin{tabular}{llll|} 
& & \\
\end{tabular} & 1 synchronous & $1+k_{m}^{2}$ & $\beta_{o p t}=\frac{4 k_{m}^{2} Q-\pi \sqrt{1+k_{m}^{2}}}{4 k_{m}^{2} Q+\pi \sqrt{1+k_{m}^{2}}}$ & None \\
\hline 2 & $\begin{array}{l}\text { 2-stage load } \\
\text { adaptation }\end{array}$ & FB & 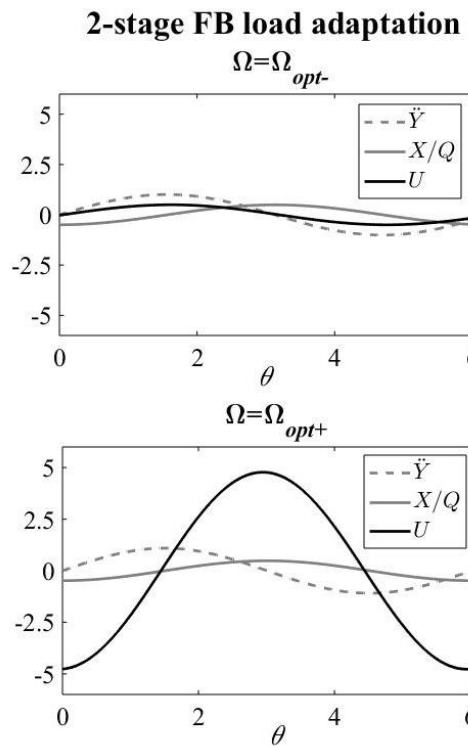 & $\begin{array}{l}1 \text { high- } \\
\text { frequency }\end{array}$ & $1+\frac{k_{m}^{2}}{2} \pm \frac{k_{m}^{2}}{2}$ & $r_{o p t \pm} \simeq \frac{k_{m}^{2} Q \pm 2 \sqrt{k_{m}^{4} Q^{2}-2\left(2+k_{m}^{2}\right)}}{2\left(1+k_{m}^{2}\right)}$ & 2 \\
\hline
\end{tabular}




\begin{tabular}{|c|c|c|c|c|c|c|c|}
\hline & & 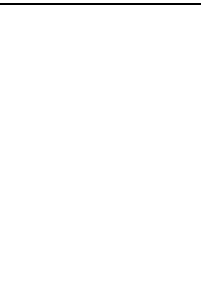 & 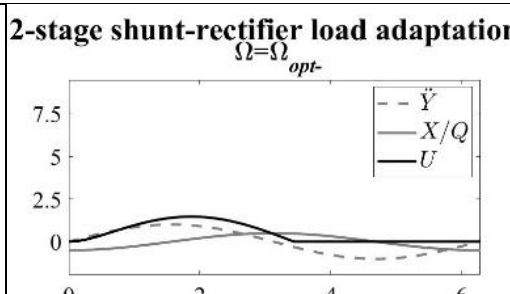 & & $\begin{array}{l}1 \\
+\frac{k_{m}^{2}}{2}\end{array}$ & 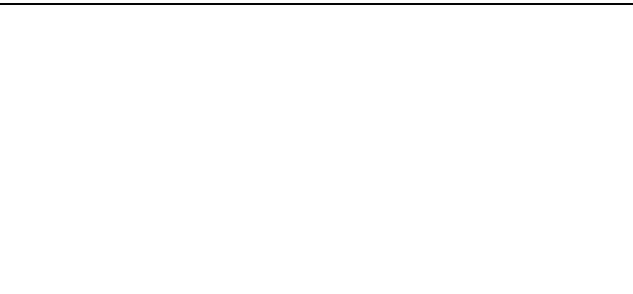 & \\
\hline 2 & $\begin{array}{l}\text { 2-stage load } \\
\text { adaptation }\end{array}$ & Shunt-rectifier & 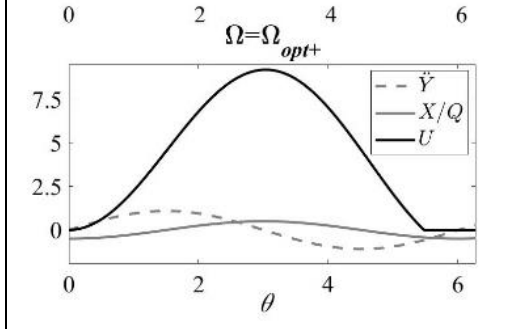 & $\begin{array}{l}1 \text { high- } \\
\text { frequency }\end{array}$ & $\pm \sqrt{1-\frac{1}{k_{m}^{2} Q} \sqrt{1+\frac{k_{m}^{2}}{2}} \pi}$ & $r_{o p t \pm}$ obtained only with numerical computation & $\frac{3.4 \pi}{4}$ \\
\hline 2 & SECE & Tunable & C: & 1 synchronous & $1+k_{m}^{2}$ & $\beta_{o p t}=\frac{4 k_{m}^{2} Q-\pi \sqrt{1+k_{m}^{2}}}{4 k_{m}^{2} Q+\pi \sqrt{1+k_{m}^{2}}}$ & None \\
\hline
\end{tabular}




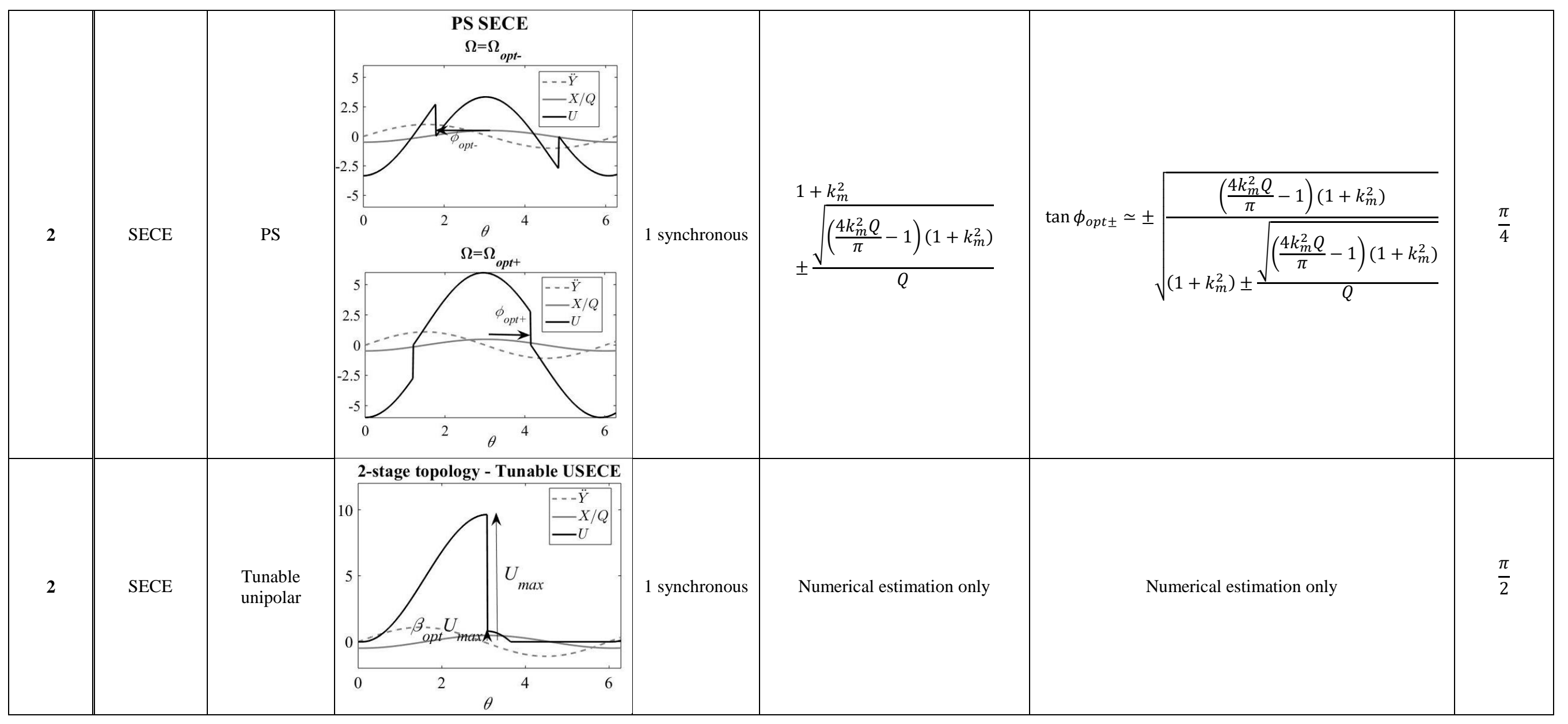




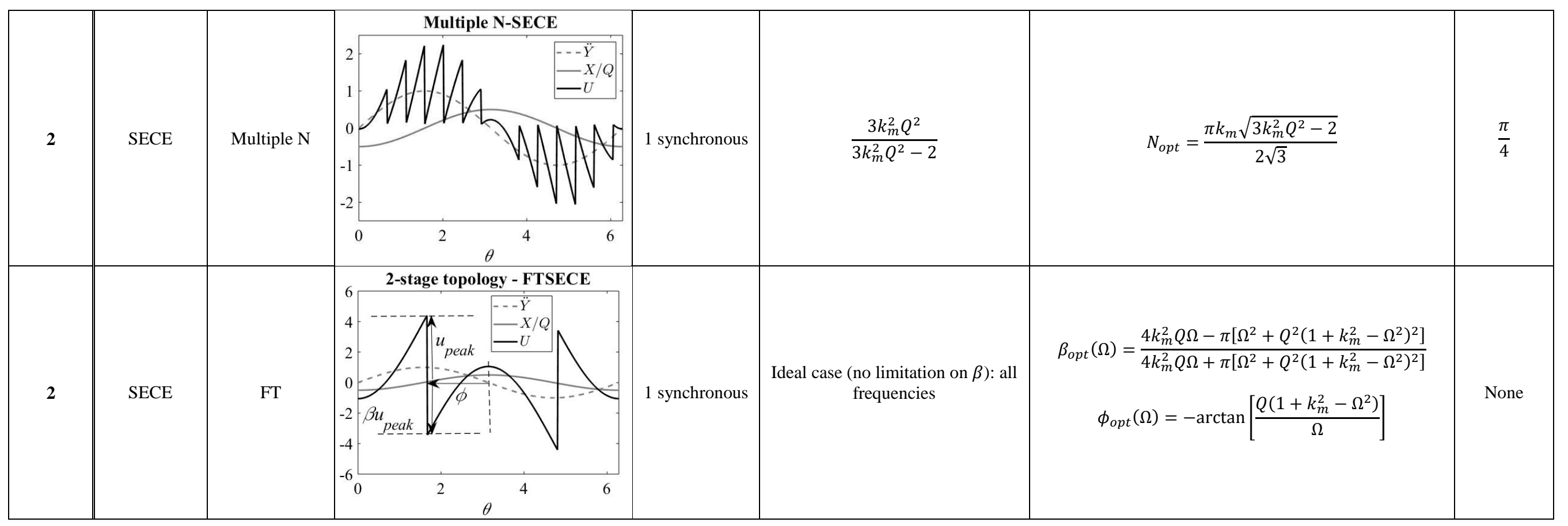




\begin{tabular}{|c|c|c|c|c|c|c|c|}
\hline 3 & $\begin{array}{l}\text { 3-stage load } \\
\text { adaptation }\end{array}$ & FB & 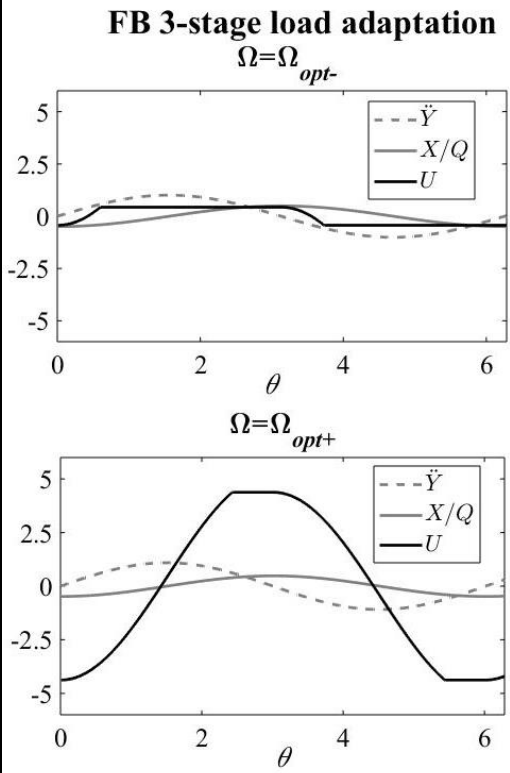 & $\begin{array}{l}1 \text { high- } \\
\text { frequency }\end{array}$ & $\begin{array}{l}+\frac{k_{m}^{2}}{2}[1 \\
\left. \pm \sqrt{1-\frac{1}{k_{m}^{2} Q} \sqrt{1+\frac{k_{m}^{2}}{2}} \pi}\right]\end{array}$ & $r_{o p t \pm}$ see (53) & $\pi$ \\
\hline 3 & $\begin{array}{l}\text { 3-stage load } \\
\text { adaptation }\end{array}$ & HB & 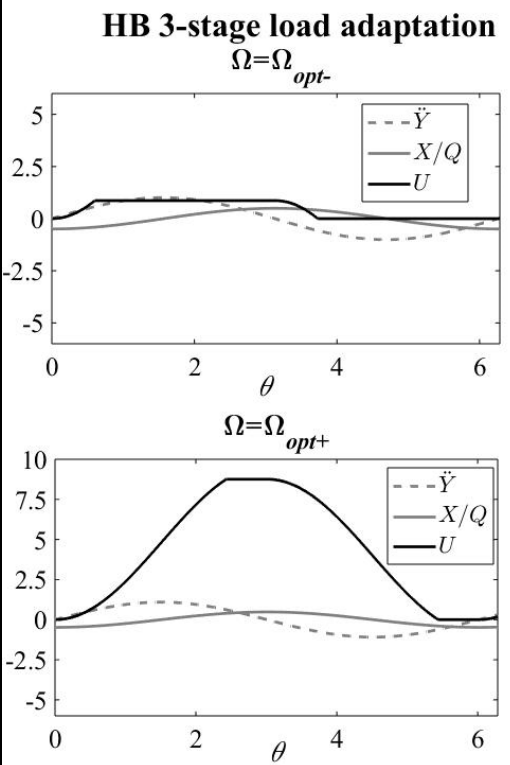 & $\begin{array}{c}1 \text { high- } \\
\text { frequency }\end{array}$ & $\begin{array}{l}1 \\
+\frac{k_{m}^{2}}{2}[1 \\
\left. \pm \sqrt{1-\frac{1}{k_{m}^{2} Q} \sqrt{1+\frac{k_{m}^{2}}{2}} \pi}\right]\end{array}$ & 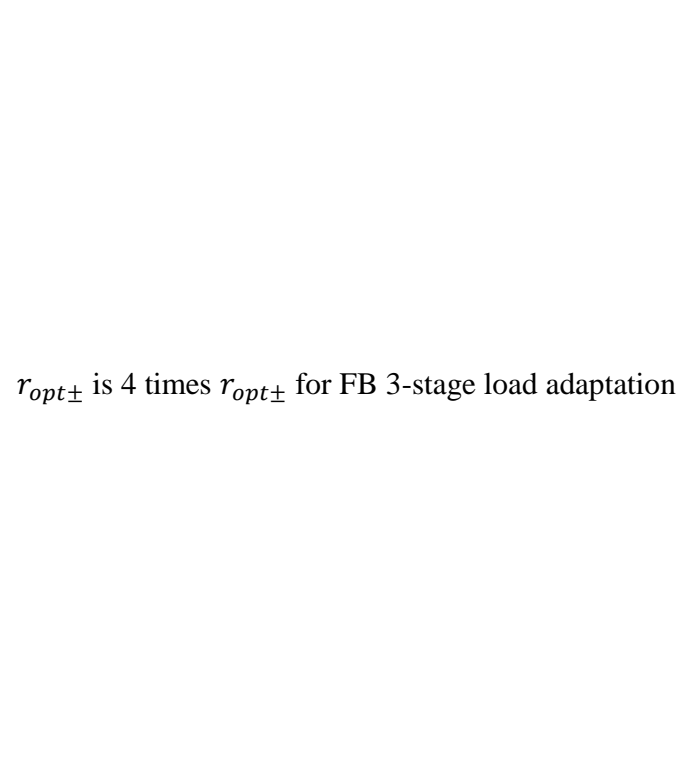 & $\pi$ \\
\hline
\end{tabular}




\begin{tabular}{|c|c|c|c|c|c|c|c|}
\hline 3 & $\begin{array}{l}\text { Hybrid } \\
\text { SECE }\end{array}$ & $\mathrm{SC}$ & 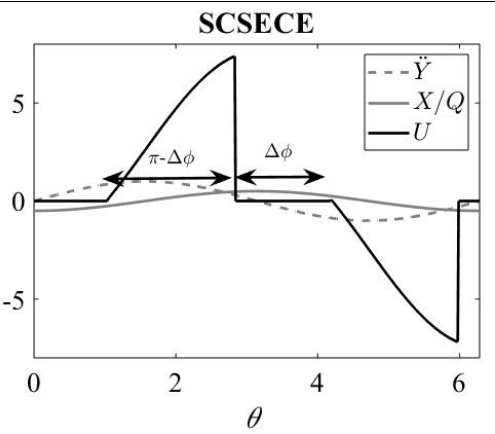 & 2 synchronous & Numerical computation only & Numerical computation only & $\frac{\pi}{4}$ \\
\hline 4 & SSH & Parallel SSHI & 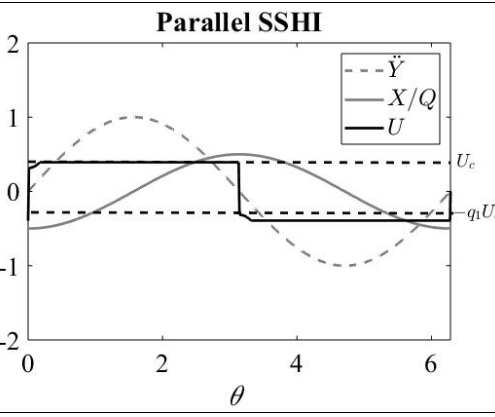 & $\begin{array}{c}1 \text { synchronous } \\
1 \text { high- } \\
\text { frequency }\end{array}$ & $\begin{array}{l}1 \\
+\frac{k_{m}^{2}}{1-q_{1}}[1 \\
\left.-\sqrt{1-\frac{\pi}{4 k_{m}^{2} Q}\left(1-q_{1}\right)^{2}}\right]\end{array}$ & $\begin{array}{l}\text { Ideal : } q_{1}=1 \text { and } r_{o p t} \simeq \frac{\pi^{2}}{8 k_{m}^{2} Q} \\
\quad \text { For } q_{1} \neq 1, \text { see }(60)\end{array}$ & $\frac{\pi\left(1-q_{1}\right)^{2}}{4}$ \\
\hline 4 & SSH & $\begin{array}{c}\text { Series SSHI } \\
\text { or DSSH or } \\
\text { ESSH or } \\
\text { SSHC }\end{array}$ & 2 & $\begin{array}{l}1 \text { synchronous } \\
1 \text { high- } \\
\text { frequency }\end{array}$ & $1+k_{m}^{2}$ & $\begin{array}{l}\text { Ideal }: q_{1}=1 \text { and } r_{\text {opt }}=\frac{2 k_{m}^{2} Q}{1+k_{m}^{2}} \\
\text { For } q_{1} \neq 1 \text {, see }(66) \\
\text { with } q_{1}=\frac{U_{m}-U_{c}}{U_{\text {peak }}+U_{c}}\end{array}$ & $\frac{\pi\left(1-q_{1}\right)}{4\left(1+q_{1}\right)}$ \\
\hline
\end{tabular}




\subsection{Technical guidelines for the choice of an adequate circuit}

As stated previously, this paper deals with the maximization of the input power, i.e. the power extracted from the piezoelectric generator for each existing technique. However, the overall performance of a piezoelectric energy harvester is also dependent on the power conversion efficiency of the harvesting circuit. This efficiency is extremely dependent on practical issues concerning the implementation and the unideal behavior of electronic components (diode conduction losses, copper and iron losses in inductors, resistive losses in the circuit, dielectric losses in the piezoelectric layer etc.). For this reason, it is very hard, if possible, to generalize about the output power that a given technique would provide without performing dedicated electrical simulations. Keeping in mind the previous statement, one may still make some general remarks, given in the following subsections.

\subsubsection{Optimization of power efficiency}

In terms of power efficiency, techniques based on a shunt rectifier minimize conduction losses compared to half-bridge or full-bridge rectifiers. Some techniques like series SSHI, SCSECE and tunable USECE exhibit higher peak voltages at maximum power point, as can be seen from Table III, which also diminishes the impact of the conduction losses. Indeed, the impact of voltage drop across diodes and other components is then reduced. This is also true for one of the two optimal power points (at the highest frequency) of HB 3-stage load adaptation, of shuntrectifier 2-stage load adaptation and of PSSECE.

\subsubsection{Considerations about the design complexity}

In terms of circuit implementation, small inductance values tend to dissipate less power than large ones. Unfortunately, a drawback of small inductances in SECE and SSH architectures is that they require the control circuit to react faster, which makes the control more complex.

\subsubsection{Specific considerations for strongly-coupled generator}

With strongly-coupled generators $\left(k_{m}^{2} Q>\pi\right)$, all aforementioned adaptive techniques are able to reach the maximum power point. Moreover, such generators tend to exhibit higher piezoelectric voltages, which tend to improve the power conversion efficiency due to less conduction losses. However, with such generators, parasitic resonance modes are more prone to actuation. Hence, the generator must be designed to minimize the actuation of higher resonance modes [108]. If designers make no effort in that sense, load adaptation techniques (2-stage or 3-stage) are less at risk. However, one should keep in mind that the performance of load adaptation techniques is quite poor in terms of bandwidth compared to other techniques (like FTSECE) [47].

5.2.4. When opting for load adaptation techniques, how to choose between 2-stage and 3stage load adaptation?

Between 2-stage and 3-stage load adaptation, the former reaches the maximum power point for weaker coupling than the latter (see corresponding FOM limit). From this perspective, the 2stage topology is preferred for generators with medium coupling $\left(2 \leq k_{m}^{2} Q<\pi\right)$. For strong coupling $\left(k_{m}^{2} Q \geq \pi\right)$, both solutions extract the maximum power but the overall performance of 3-stage load adaptation tends to be better. Indeed, the presence of a smoothing capacitor improves the power efficiency of the DC-DC converter (stable voltage at its input). For weaker 
coupling $\left(k_{m}^{2} Q<2\right)$, load adaptation techniques do not reach the maximum power but 2-stage load adaptation tends to harvest more power than 3-stage load adaptation, as long as the power conversion efficiency of the DC-DC converter remains decent.

\subsubsection{Remarks about the impact of the vibration frequency}

As a last remark, one may wonder about the feasibility of these techniques with respect to the mechanical oscillation frequency. In fact, all the aforementioned techniques are usable in common $\mathrm{PEH}$, whether they reach over $100 \mathrm{~Hz}$, or ultra-low frequencies (lower than $10 \mathrm{~Hz}$ ), as long as the components are appropriate.

\subsubsection{Final remarks about qualitative statements}

All these remarks are only general tendencies. Like all qualitative statements, they tend to be relative and rough, if not imprecise. The choice of an adequate circuit still lies on a thorough analysis and tradeoff between all the pros and cons (maximizing input power, maximizing power conversion efficiency and/or minimizing the size of the circuit, the cost and the design complexity). This paper should help designers to ponder the advantages and drawbacks of each available choice.

\section{Conclusion}

In this paper, we have reviewed and categorized existing vibration energy harvesting techniques for piezoelectric generators. An analytical study based on previous literature has allowed us to explicit general conditions that such a system must fulfill to reach the maximum available power. In terms of implementation, the number of stages (and especially control stages) affects the complexity of electronic architectures. For this reason, we have proposed a hierarchy of techniques, taking into account the number of optimization parameters available, the topology (arrangement of functional blocs), the architecture (implementation of the blocs) and the control technique (waveforms). Following this hierarchical description, we have illustrated our theoretical results on numerous techniques from the scientific literature.

Based on this review, authors of new harvesting techniques can find their way among existing topologies and architectures and find appropriate denominations for their inventions, avoiding the multiplication of new acronyms. We hope that MPPT algorithms will be developed and implemented for the existing techniques. We also hope to perform comparative studies of these algorithms for piezoelectric energy harvesters, as other fields have carried out (such as photovoltaic energy harvesters [109]).

\section{References}

[1] K. V. Selvan and M. Ali, "Micro-scale energy harvesting devices: Review of methodological performances in the last decade," Renewable and Sustainable Energy Reviews, vol. 54, p. 1035-1047, 2016.

[2] E. Arroyo, A. Badel, F. Formosa, Y. Wu and J. Qiu, "Comparison of electromagnetic and piezoelectric vibration energy harvesters: model and experiments," Sensors Actuators A: Physical, vol. 183, pp. 148-156, 2012.

[3] X. Wang, S. John, S. Watkins, X. Yu, H. Xiao, X. Liang and H. Wei, "Similarity and duality of electromagnetic and piezoelectric vibration energy harvesters," Mechanical Systems and Signal Processing, vol. 1, pp. 53-53, 2014. 
[4] A. Toprak and O. Tigli, "Piezoelectric energy harvesting: State-of-the-art and challenges," Applied Physics Reviews, vol. 1, p. 031104, 2014.

[5] M. Safaei, H. A. Sodano and S. R. Anton, "A review of energy harvesting using piezoelectric materials: State-of-theart a decade later (2008-2018)," Smart Materials and Structures, vol. 28, no. 11, p. 113001, 2019.

[6] J. Dicken, P. Mitcheson, I. Stoianov and E. Yeatman, "Power-Extraction Circuits for Piezoelectric Energy Harvesters in Miniature and Low-Power Applications," IEEE Transactions on Power Electronics, vol. 27, no. 11, pp. 4514 - 4529, 2012.

[7] F. G. Dell'Anna, T. Dong, P. Li, Y. Wen, Z. Yang, M. R. Casu, M. Azadmehr and Y. Berg, "State-of-the-Art Power Management Circuits for Piezoelectric Energy Harvesters," IEEE Circuits and Systems Magazine, vol. 18, no. 3, pp. 27-48, 2018.

[8] E. Halvorsen, C. P. Le, P. D. Mitcheson and E. M. Yeatman, "Architecture-independent power bound for vibration energy harvesters," in PowerMEMS, London, UK, 2013.

[9] A. H. Hosseinloo and K. Turitsyn, "Fundamental limits to nonlinear energy harvesting," Phys. Rev. Applied, vol. 4, p. 064009, 2015.

[10] Y. Liao and H. Sodano, "Optimal power, power limit and damping of vibration based piezoelectric power harvesters," Smart Materials and Structures, vol. 27, no. 7, p. 075057-12, 2018.

[11] D. Hawes and R.S.Langley, "Limits on the power available to harvest from broadband random excitation," Journal of Sound and Vibration, vol. 399, pp. 244-256, 2017.

[12] A. Erturk and D. J. Inman, "A distributed parameter electromechanical model for cantilevered piezoelectric energy harvesters," ASME J. Vib. Acoust., vol. 130, p. 041002 , 2008.

[13] H. Zhang, L. R. Corr and T. Ma, "Effects of electrical loads containing non-resistive components on piezoelectric energy harvesting," Mechanical Systems and Signal Processing, vol. 111, pp. 210-221, 2018.

[14] Y. K. Ramadass and A. P. Chandrakasan, "An Efficient Piezoelectric Energy Harvesting Interface Circuit Using a Bias-Flip Rectifier and Shared Inductor," IEEE Journal of Solid-State Circuits, vol. 45, no. 1, pp. 189-204, January 2010.

[15] D. Kwon and G. A. Rincon-Mora, "A rectifier-free piezoelectric energy harvester circuit," in Circuits and Systems, 2009. ISCAS 2009. IEEE International Symposium on, Taipei, 2009.

[16] G. K. Ottman, H. F. Hofmann, A. C. Bhatt and G. A. Lesieutre, "Adaptive piezoelectric energy harvesting circuit for wireless remote power supply," IEEE Transactions on Power Electronics, vol. 17, no. 5, pp. 669-676, September 2002.

[17] G. K. Ottman, H. F. Hofmann and G. A. Lesieutre, "Optimized Piezoelectric Energy Harvesting Circuit Using Step-Down Converter in Discontinuous Conduction Mode," IEEE Transactions on Power Electronics, vol. 18, no. 2, pp. 696-703, March 2003.

[18] S. Stanzione, C. Van Liempd, R. Van Schaijk, Y. Naito, R. F. Yazicioglu and C. Van Hoof, "A Self-Biased 5-to-60V Input Voltage and 25-to-1600 $\mu \mathrm{W}$ Integrated DC-DC Buck Converter with Fully Analog MPPT Algorithm Reaching up to 88\% End-to-End Efficiency," in ISSCC, San Francisco, 2013.

[19] A. Badel and E. Lefeuvre, "Nonlinear Conditioning Circuits for Piezoelectric Energy Harvesters," in Nonlinearity in Energy Harvesting Systems, E. Blokhina, A. E. Aroudi, E. Alarcon and D. Galayko, Eds., Cham, Springer, 2016. 
[20] C. Richard, D. Guyomar, D. Audigier and H. Bassaler, "Enhanced semi-passive damping using continuous switching of a piezoelectric device on an inductor," in Smart Structures and Materials 2000: Damping and Isolation, Newport Beach, CA, 2000.

[21] E. Lefeuvre, A. Badel, C. Richard and D. Guyomar, "Piezoelectric energy harvesting device optimization by synchronous electric charge extraction," J. of Intelligent Material Systems and Structures, vol. 16, pp. 865-876, 2005.

[22] C. Richard, D. Guyomar, D. Audigier and G. Ching, "Semi passive damping using continuous switching of a piezoelectric device," in Proc. SPIE Int. Symp. Smart Struct. Mater., San Diego, CA, 1998.

[23] A. Richter, A. Strobel, N. Joram, F. Ellinger, L. Göpfert and R. Marg, "Tunable Interface for Piezoelectric Energy Harvesting," in Systems, Signals \& Devices (SSD), 2014 11th International Multi-Conference on, Barcelona, 2014.

[24] E. Lefeuvre, A. Badel, A. Brenes, S. Seok, M. Woytasik and C.-S. Yoo, "Analysis of piezoelectric energy harvesting system with tunable SECE interface," Smart Materials and Structures, vol. 26, no. 3, February 2017.

[25] E. Lefeuvre, A. Badel, A. Brenes, S. Seok and C.-S. Yoo, "Power and frequency bandwidth improvement of piezoelectric energy harvesting devices using phase-shifted SECE interface circuit," J. of Intelligent Material Systems and Structures, 2017.

[26] D. Guyomar, A. Badel, E. Lefeuvre and C. Richard, "Toward Energy Harvesting Using Active Materials and Conversion Improvement by Nonlinear Processing," IEEE Transactions on ultrasonics, ferroelectrics and frequency control, vol. 52, no. 4, pp. 584-595, 2005.

[27] S. Ben-Yaakov and N. Krihely, "Resonant rectifier for piezoelectric sources," in Twentieth Annual IEEE Applied Power Electronics Conference and Exposition (APEC), Austin, TX, 2005.

[28] A. Badel, A. Benayad, E. Lefeuvre, L. Lebrun, C. Richard and D. Guyomar, "Single Crystals and Nonlinear Process for Outstanding Vibration-Powered Electrical Generators," IEEE Transactions on Ultrasonics, Ferroelectrics and Frequency Control, vol. 53, no. 4, pp. 673-684, 2006.

[29] E. Lefeuvre, A. Badel, C. Richard, L. Petit and D. Guyomar, "A comparison between several vibration-powered piezoelectric generators for standalone systems," Sensors and Actuators A, vol. 126, pp. 405-416, 2006.

[30] P.-H. Hsieh, C.-H. Chen and H.-C. Chen, "Improving the Scavenged Power of Nonlinear Piezoelectric Energy Harvesting Interface at Off-Resonance by Introducing Switching Delay," IEEE Transactions on Power Electronics, vol. 30, no. 6, pp. 3142-3155, 2015.

[31] S. Lu and F. Boussaid, "A Highly Efficient P-SSHI Rectifier for Piezoelectric Energy Harvesting," IEEE Transactions on Power Electronics, vol. 30, no. 10, pp. 5364 - 5369, 2015.

[32] S. Du and A. A. Seshia, "An Inductorless Bias-Flip Rectifier for Piezoelectric Energy Harvesting," IEEE Journal of Solid-State Circuits, vol. 52, no. 10, pp. 2746-2757, 2017.

[33] Y. Ammar and S. Basrour, "Non-linear techniques for increasing harvesting energy from piezoelectric and electromagnetic micro-powergenerators," in DTIP, Stresa, Lago Maggiore, Italy, 2006.

[34] S. Du, Y. Jia, C. Zhao, G. A. J. Amaratunga and A. A. Seshia, "A Fully Integrated SplitElectrode SSHC Rectifier for Piezoelectric Energy Harvesting," IEEE Journal of SolidState Circuits, vol. 54, no. 6, pp. 1733 - 1743, 2019. 
[35] T. Hehn, F. Hagedorn, D. Maurath, D. Marinkovic, I. Kuehne, A. Frey and Y. Manoli, "A Fully Autonomous Integrated Interface Circuit for Piezoelectric Harvesters," IEEE Journal of Solid-State Circuits, vol. 47, no. 9, pp. 2185 - 2198, 2012.

[36] M. K. Kazimierczuk, "Analysis of Class E Zero-Voltage-Switching Rectifier," IEEE Transactions on Circuits and Systems, vol. 37, no. 6, pp. 747-755, June 1990.

[37] A. Brenes, E. Lefeuvre, A. Badel, S. Seok and C.-S. Yoo, "Shunt-diode rectifier: a new scheme for efficient low-voltage piezoelectric energy harvesting," Smart Materials and Structures, vol. 28, no. 1, 2018.

[38] A. Brenes, E. Lefeuvre, A. Badel, S. Seok and C.-S. Yoo, "Unipolar synchronized electric charge extraction for piezoelectric energy harvesting," Smart Materials and Structures, 2018.

[39] L. Garbuio, M. Lallart, D. Guyomar, C. Richard and D. Audigier, "Mechanical Energy Harvester With Ultralow Threshold Rectification Based on SSHI Nonlinear Technique," IEEE Transactions on Industrial Electronics, vol. 56, no. 4, pp. 1048-1056, April 2009.

[40] Y. Wu, A. Badel, F. Formosa, W. Liu and A. E. Agbossou, "Piezoelectric vibration energy harvesting by optimized synchronous electric charge extraction," Journal of Intelligent Material Systems and Structures, vol. 24, no. 12, pp. 1445-1458, 2013.

[41] H. Xia, Y. Xia, Y. Ye, L. Qian, G. Shi and R. Chen, "Analysis and Simulation of Synchronous Electric Charge Partial Extraction Technique for Efficient Piezoelectric Energy Harvesting," IEEE Sensors Journal , vol. 18, no. 15, pp. 6235 - 6244, 2018.

[42] C. S. Kong, "A general maximum power transfer theorem," IEEE Transactions on Education, vol. 38, no. 3, pp. 296-298, 1995.

[43] Y. Cai and Y. Manoli, "A Piezoelectric Energy-Harvesting Interface Circuit with Fully Autonomous Conjugate Impedance Matching, 156\% Extended Bandwidth, and $0.38 \mu \mathrm{W}$ Power Consumption," in ISSCC, San Francisco, 2018.

[44] W. Liu, C. Zhao, A. Badel, F. Formosa, Q. Zhu and G. Hu, "Compact self-powered synchronous energy extraction circuit design with enhanced performance," Smart Materials and Structures, vol. 27, p. 047001, 2018.

[45] B. Zhao and J. Liang, "On the Circuit Solutions towards Broadband and High-Capability Piezoelectric Energy Harvesting Systems," in Proceedings of Active and Passive Smart Structures and Integrated Systems XII, Denver, CO, 2018.

[46] C. Chen, K. Zhao and J. Liang, "Impedance Analysis of Piezoelectric Energy Harvesting System Using Synchronized Charge Extraction Interface Circuit," in Active and Passive Smart Structures and Integrated Systems, Portland, OR, 2017.

[47] A. Brenes, E. Lefeuvre and C.-S. Yoo, "Experimental validation of wideband piezoelectric energy harvesting based on frequency-tuning synchronized charge extraction," Journal of Physics : Conference Series, vol. 1052, p. 012050, 2018.

[48] P. Gasnier, J. Willemin, S. Boisseau, G. Despesse, C. Condemine, G. Gouvernet and J.J. Chaillout, "An Autonomous Piezoelectric Energy Harvesting IC based on a Synchronous Multi-Shots Technique," in ESSCIRC, Bucharest, Romania, 2013.

[49] P. Gasnier, J. Willemin, S. Boisseau, G. Despesse, C. Condemine, G. Gouvernet and J.J. Chaillout, "An Autonomous Piezoelectric Energy Harvesting IC based on a Synchronous Multi-Shots Technique," IEEE Journal of Solid-State Circuits, vol. 49, no. 7, pp. 1561-2014, July 2014.

[50] A. Morel, A. Badel, Y. Wanderoild and G. Pillonnet, "A unified N-SECE strategy for highly coupled piezoelectric energy scavengers," Smart Materials and Structures, vol. 27, p. 084002, 2018. 
[51] A. Brenes, E. Lefeuvre, S. Seok and C.-S. Yoo, "Tunable unipolar synchronized electric charge extraction strategy for piezoelectric energy harvesting," Journal of Intelligent Material Systems and Structures, 2019.

[52] M. Lallart, W.-J. Wu, Y. Hsieh and L. Yan, "Synchronous inversion and charge extraction (SICE): a hybrid switching interface for efficient vibrational energy harvesting," Smart Materials and Structures, vol. 26, p. 115012, 2017.

[53] A. Morel, G. Pillonnet, P. Gasnier, E. Lefeuvre and A. Badel, "Frequency Tuning of Piezoelectric Energy Harvesters Thanks to a Short-Circuit Synchronous Electric Charge Extraction," Smart Materials and Structures, vol. 28, no. 2, p. 025009, 2018.

[54] M. Lallart, L. Garbuio, L. Petit, C. Richard and D. Guyomar, "Double Synchronized Switch Harvesting (DSSH): A New Energy Harvesting Scheme for Efficient Energy Extraction," IEEE Transactions on Ultrasonics, Ferroelectrics and Frequency Control, vol. 55, no. 10, pp. 2119-2008, 2008.

[55] H. Shen, J. Qiu, H. Ji, K. Zhu and M. Balsi, "Enhanced synchronized switch harvesting: a new energy harvesting scheme for efficient energy extraction," Smart Materials and Structures, vol. 19, no. 11, p. 115017, 2010.

[56] C. Cheng, Z. Chen, Y. Xiong, H. Shia and Y. Yang, "A high-efficiency, self-powered nonlinear interface circuit for bi-stable rotating piezoelectric vibration energy harvesting with nonlinear magnetic force," International Journal of Applied Electromagnetics and Mechanics, vol. 51, p. 235-248, 2016.

[57] S.-C. Kwon, J. Onoda and H.-U. Oh, "Performance evaluation of a novel piezoelectricbased high-frequency surge-inducing synchronized switching strategy for micro-scale energy harvesting," Mechanical Systems and Signal Processing, vol. 117, pp. 361-382, 2019.

[58] Z. Chen, J. He, J. Liu and Y. Xiong, "Switching Delay in Self-powered Nonlinear Piezoelectric Vibration Energy Harvesting Circuit: Mechanism, Effects and Solution," IEEE Transactions on Power Electronics, vol. 34, no. 3, pp. 2427 - 2440, 2018.

[59] Y. Zhao and J. Liang, "Synchronized Triple Bias-Flip Circuit for Piezoelectric Energy Harvesting Enhancement: Operation Principle and Experimental Validation," in IEEE Energy Conversion Congress and Exposition, Milwaukee, WI, 2016.

[60] B. Zhao, J. Liang and K. Zhao, "Phase-Variable Control of Parallel Synchronized Triple Bias-Flips Interface Circuit towards Broadband Piezoelectric Energy Harvesting," in IEEE International Symposium on Circuits and Systems, Florence, Italy, 2018.

[61] M. Lallart and D. Guyomar, "An optimized self-powered switching circuit for non-linear energy harvesting with low voltage output," Smart Materials and Structures, vol. 17, no. 3, pp. 3503-3511, 2008.

[62] M. Lallart, W.-J. Wu, L. Yan and S.-W. Hung, "Inductorless Synchronized Switch Harvesting using a Piezoelectric Oscillator," IEEE Transactions on Power Electronics, p. Early access, 2019.

[63] W. Liu, G. Qin and Q. Zhu, "Synchronous extraction circuit with self-adaptive peakdetection mechanical switches design for piezoelectric energy harvesting," Applied Energy, vol. 230, pp. 1292-1303, 2018.

[64] M. Lallart, "Nonlinear technique and self-powered circuit for efficient piezoelectric energy harvesting under unloaded cases," Energy Conversion and Management, vol. 133, p. 444-457, 2017. 
[65] J. Liang and W.-H. Liao, "Impedance Modeling and Analysis for Piezoelectric Energy Harvesting Systems," IEEE/ASME Transactions on Mechatronics, vol. 17, no. 6, pp. 1145-1157, December 2012.

[66] Y. Liao and J. Liang, "Maximum power, optimal load, and impedance analysis of piezoelectric vibration energy harvesters," Smart Materials and Structures, vol. 27, p. 075053, 2018.

[67] N. Kong, D. S. Ha, A. Erturk and D. J. Inman, "Resistive Impedance Matching Circuit for Piezoelectric Energy Harvesting," Journal of Intelligent Material Systems and Structures, vol. 21, pp. 1293-1302, 2010.

[68] H. S. Kim, J.-H. Kim and J. Kim, "A Review of Piezoelectric Energy Harvesting Based on Vibration," International Journal of Precision Engineering and Manufacturing, vol. 12, no. 6, pp. 1129-1141, December 2011.

[69] D. Kwon and G. A. Rincón-Mora, "A 2- $\mu \mathrm{m}$ BiCMOS Rectifier-Free AC-DC Piezoelectric Energy Harvester-Charger IC," IEEE Transactions on biomedical circuits and systems, vol. 4, no. 6, pp. 400-409, 2010.

[70] Y. C. Shu and I. C. Lien, "Efficiency of energy conversion for a piezoelectric power harvesting system," Journal of Micromechanics and Microengineering, vol. 16, pp. 2429-2438, 2006.

[71] H. Hu, H. Xue and Y. Hu, "A Spiral-Shaped Harvester with an Improved Harvesting Element and an Adaptive Storage Circuit," IEEE Transactions on Ultrasonics, Ferroelectrics and Frequency Control, vol. 54, no. 6, pp. 1177-1187, 2007.

[72] I. C. Lien, Y. C. Shu, W. J. Wu, S. M. Shiu and H. C. Lin, "Revisit of series-SSHI with comparisons to other interfacing circuits in piezoelectric energy harvesting," Smart Materials and Structures, vol. 19, 2010.

[73] M. Lallart, L. Garbuio, C. Richard and D. Guyomar, "Low-cost capacitor voltage inverter for outstanding performance in piezoelectric energy harvesting," IEEE Transactions on Ultrasonics, Ferroelectrics, and Frequency Control, vol. 57, no. 2, pp. 281 - 291, 2010.

[74] S. Javvaji, V. Singhal, V. Menezes, R. Chauhan and S. Pavan, "Multi-Step Bias-Flip Rectification for Piezoelectric Energy Harvesting," in IEEE 44th European Solid State Circuits Conference (ESSCIRC), Dresden, Germany, 2018.

[75] S. Du, G. A. J. Amaratunga and A. A. Seshia, "A Cold-Startup SSHI Rectifier for Piezoelectric Energy Harvesters With Increased Open-Circuit Voltage," IEEE Transactions on Power Electronics, vol. 34, no. 1, pp. 263-274, 2019.

[76] D. A. Hall, "Review - Nonlinearity in piezoelectric ceramics," Journal of Material Science, vol. 36, no. 19, p. 4575-4601, October 2001.

[77] A. Erturk and D. J. Inman, "Issues in mathematical modeling of piezoelectric energy harvesters," Smart Materials and Structures, vol. 17, p. 065016, 2008.

[78] Y. Liao and H. A. Sodano, "Model of a single mode energy harvester and properties for optimal power generation," Smart Materials and Structures, vol. 17, p. 065026, November 2008.

[79] N. E. duToit, B. L.Wardle and S.-G. Kim, "Design considerations for MEMS-scale piezoelectric mechanical vibration energy harvesters," Integrated Ferroelectrics, vol. 71, pp. 121-160, 2005.

[80] V. Kovacova, "Study of correlations between microstructure and piezoelectric properties of PZT thin films," Université Grenoble Alpes, 2015. 
[81] R. Ramlan, M. Brennan, B. Mace and I. Kovacic, "Potential benefits of a non-linear stiffness in an energy harvesting device," Nonlinear Dynamics, vol. 59, pp. 545-558, 2010.

[82] N. J. Guilar, R. Amirtharajah and P. J. Hurst, "A Full-Wave Rectifier With Integrated Peak Selection for Multiple Electrode Piezoelectric Energy Harvesters," IEEE Journal of Solid-State Circuits, vol. 44, no. 1, pp. 240-246, 2009.

[83] M. Lallart and D. Guyomar, "Piezoelectric conversion and energy harvesting enhancement by initial energy injection," Applied Physics Letters, vol. 97, no. 1, p. 014104, 2010.

[84] D. Guyomar and M. Lallart, "Recent Progress in Piezoelectric Conversion and Energy Harvesting Using Nonlinear Electronic Interfaces and Issues in Small Scale Implementation," Micromachines, vol. 2, pp. 274-294, 2011.

[85] R. D'hulst, P. D. Mitcheson and J. Driesen, "CMOS Buck-Boost Power processing circuitry for powerMEMS generators," in PowerMEMS, Berkeley, 2006.

[86] H. Xia, R. Chen, L. Ren and Q. Zhou, "Direct calculation of source impedance to adaptive maximum power point tracking for broadband vibration," Journal of Intelligent Material Systems and Structures, September 2016.

[87] R. D'hulst, T. Sterken, R. Puers, G. Deconinck and J. Driesen, "Power Processing Circuits for Piezoelectric Vibration-Based Energy Harvesters," IEEE Transactions on Industrial Electronics, vol. 57, no. 12, pp. 4170-4177, 2010.

[88] Y. C. Shu and I. C. Lien, "Analysis of power output for piezoelectric energy harvesting systems," Smart Materials and Structures, vol. 15, pp. 1499-1512, 2006.

[89] R. D'hulst, T. Sterken, P. Fiorini, R. Puers and J. Driesen, "Energy Scavengers : Modeling and Behavior with Different Load Circuits," in The 33rd Annual Conference of the IEEE Industrial Electronics Society (IECON), Taipei, Taiwan, 2007.

[90] W.-J. Wu, A. Wickenheiser, T. Reissman and E. Garcia, "Modeling and experimental verification of synchronized discharging techniques for boosting power harvesting from piezoelectric transducers," Smart Materials and Structures, vol. 18, no. 5, p. 055012, 2009.

[91] A. Morel, A. Quelen, P. Gasnier, R. Grézaud, S. Monfray, A. Badel and G. Pillonnet, "A Shock-Optimized SECE Integrated Circuit," IEEE Journal of Solid-State Circuits, vol. 53, no. 12, pp. 3420 - 3433, 2018.

[92] M. Meng, A. Ibrahim, T. Xue, H.-G. Yeo, D. Wang, S. Roundy, S. Trolier-McKinstry and M. Kiani, "27.4 Multi-Beam Shared-Inductor Reconfigurable Voltage/SECE-Mode Piezoelectric Energy Harvesting of Multi-Axial Human Motion," in IEEE International Solid-State Circuits Conference - (ISSCC), San Francisco, CA, 2019.

[93] A. Shareef, W.-L. Goh, S. Narasimalu and Y. Gao, "A Rectifier-less AC-DC Interface Circuit for Ambient Energy Harvesting from Low-Voltage Piezoelectric Transducer Array," IEEE Transactions on Power Electronics, vol. 34, no. 2, pp. 1446 - 1457, 2019.

[94] M. Dini, A. Romani, M. Filippi and M. Tartagni, "A Nano-Power Synchronous Charge Extractor IC for Low Voltage Piezoelectric Energy Harvesting with Residual Charge Inversion," IEEE Transactions on Power Electronics, vol. 31, no. 2, pp. 1263-1274, January 2015.

[95] S. Chamanian, A. Muhtaroglu and H. Kulah, "A self-adapting synchronized-switch interface circuit for piezoelectric energy harvesters," IEEE Transactions on Power Electronics, Vols. Early-access, 2019. 
[96] S. Fan, R. Wei, L. Zhao, X. Yang, L. Geng and P.-X.-L. Feng, "An Ultralow Quiescent Current Power Management System With Maximum Power Point Tracking (MPPT) for Batteryless Wireless Sensor Applications," IEEE Transactions on Power Electronics, vol. 33, no. 9, pp. 7326 - 7337, 2017.

[97] Y.-J. Hu, I.-C. Chen and T.-H. Tsai, "A Piezoelectric Vibration Energy Harvesting System with Improved Power Extraction Capability," in IEEE Asian Solid-State Circuits Conference, Toyama, Japan, 2016.

[98] G. Shi, Y. Xia, H. Xia, X. Wang, L. Qian and Z. Chen, "An Efficient Power Management Circuit Based on Quasi Maximum Power Point Tracking With Bidirectional Intermittent Adjustment for Vibration Energy Harvesting," IEEE Transactions on Power Electronics, vol. 34, no. 10, pp. 9671 - 9685, 2019.

[99] Y. Peng, K. D. Choo, S. Oh, I. Lee, T. Jang and Y. Kim, "An Efficient Piezoelectric Energy Harvesting Interface Circuit Using a Sense-and-Set Rectifier," IEEE Journal of Solid-State Circuits, vol. Early Access, pp. 1-14, 2019.

[100] S. Du and A. A. Seshia, "A fully integrated split-electrode synchronized-switchharvesting-on-capacitors (SE-SSHC) rectifier for piezoelectric energy harvesting with between $358 \%$ and $821 \%$ power-extraction enhancement," in IEEE International Solid - State Circuits Conference - (ISSCC), San Francisco, CA, 2018.

[101] Z. Chen, Y. Jiang, M.-K. Law, P.-I. Mak, X. Zeng and R. P. Martins, "27.3 A Piezoelectric Energy-Harvesting Interface Using Split-Phase Flipping-Capacitor Rectifier and Capacitor Reuse Multiple-VCR SC DC-DC Achieving 9.3× EnergyExtraction Improvement," in IEEE International Solid- State Circuits Conference (ISSCC), San Francisco, CA, 2019.

[102] S. Yang and G. A. Rincon-Mora, "Energy-Harvesting Piezoelectric-Powered CMOS Series Switched-Inductor Bridge," IEEE Transactions on Power Electronics, vol. 34, no. 7, pp. 6489 - 6497, 2019.

[103] A. M. Eltamaly and K. E. Addoweesh, "A Novel Self-Power SSHI Circuit for Piezoelectric Energy Harvester," IEEE Transactions on Power Electronics, vol. 32, no. 10, pp. 7663-7673, 2017.

[104] D. A. Sanchez, J. Leicht, F. Hagedorn, E. Jodka, E. Fazel and Y. Manoli, "A ParallelSSHI Rectifier for Piezoelectric Energy Harvesting of Periodic and Shock Excitations," IEEE Journal of Solid-State Circuits, vol. 51, no. 12, pp. 2867 - 2879, 2016.

[105] J. Liang, Y. Zhao and K. Zhao, "Synchronized Triple Bias-Flip Interface Circuit for Piezoelectric Energy Harvesting Enhancement," IEEE Transactions on Power Electronics, vol. 34, no. 1, pp. 275 - 286, 2019.

[106] Y. Hu, H. Xue, T. Hu and H. Hu, "Nonlinear Interface Between the Piezoelectric Harvesting Structure and the Modulating Circuit of an Energy Harvester with a Real Storage Battery," IEEE Transactions on Ultrasonics, Ferroelectrics and Frequency Control, vol. 55, no. 1, pp. 148-160, 2008.

[107] A. Morel, G. Pillonnet and A. Badel, "A Tunable Hybrid SSHI Strategy for Piezoelectric Energy Harvesting with Enhanced Off-resonance Performances," in PowerMEMS, Daytona, 2018.

[108] D. Gibus, P. Gasnier, A. Morel, S. Boisseau and A. Badel, "Modelling and design of highly coupled piezoelectric energy harvesters for broadband applications," in PowerMEMS, Daytona Beach, United States, 2018. 
[109] B. Subudhi and R. Pradhan, "A Comparative Study on Maximum Power Point Tracking Techniques for Photovoltaic Power Systems," IEEE Transactions on Sustainable Energy, vol. 4, no. 1, pp. 89 - 98, 2013. 